\title{
ANALYSIS OF A GOLDSCHMIED PROPULSOR USING COMPUTATIONAL FLUID DYNAMICS REFERENCING CALIFORNIA POLYTECHNIC'S GOLDSCHMIED PROPULSOR TESTING
}

\author{
A Thesis \\ presented to \\ the Faculty of California Polytechnic State University, \\ San Luis Obispo
}

\author{
In Partial Fulfillment \\ of the Requirements for the Degree \\ Master of Science in Aerospace Engineering
}

by

Cory A. Seubert

June 2012 
(C) 2012

Cory Alexander Seubert

ALL RIGHTS RESERVED 


\section{COMMITTEE MEMBERSHIP}

TITLE:

AUTHOR:

DATE SUBMITTED:

COMMITTEE CHAIR:

COMMITTEE MEMBER:

\begin{abstract}
Analysis of a Goldschmied Propulsor Using Computational Fluid Dynamics Referencing California Polytechnic's Goldschmied Propulsor Testing
\end{abstract}

Cory A. Seubert

June 2012

Dr. David Marshall, Instructor Faculty, Aerospace Engineering

Dr. Robert McDonald, Instructor Faculty, Aerospace Engineering

COMMITTEE MEMBER: Dr. Jin Tso, Instructor Faculty, Aerospace Engineering

COMMITTEE MEMBER: Dr. Russell Westphal, Instructor Faculty, Mechanical Engineering 


\begin{abstract}
Analysis of a Goldschmied Propulsor Using Computational Fluid Dynamics Referencing California Polytechnic's Goldschmied Propulsor Testing
\end{abstract}

Cory A. Seubert

The Goldschmied Propulsor is a concept that was introduced in mid 1950 's by Fabio Goldschmied. The concept combines boundary layer suction and boundary layer ingestion technologies to reduce drag and increase propulsor efficiency. The most recent testing, done in 1982, left questions concerning the validity of the results. To answer these questions a 38.5in Goldschmied Propulsor was constructed and tested in Cal Poly's 3x4ft wind tunnel. The focus of their wind tunnel investigation was to replicate Goldschmied's original testing and increase the knowledge base on the subject. The goal of this research was to create a computational fluid dynamics (CFD) model to help visualize the flow phenomenon and see how well CFD was able to replicate Cal Poly's wind tunnel results. CFD cases were run to get a comparison of the computational model and the wind tunnel results. For the straight tunnel geometry for the 0.385 " slot and cusp A we found a body, pressure and friction drag, fan off $C_{D}$ of 0.0526 and a fan on at 500 Pascals with a $C_{D}$ of 0.0545 . This is similar to the wind tunnel results but because of large errors in measuring overall drag we are not able to directly compare to the wind tunnel results. Overall we see that the trends match, mainly that the fan does not decrease the total pressure drag. This was a result of poor geometry and high fan speeds needed for attachment. The tested geometry is less than ideal and has a long way to go before it is of a shape that would have the potential to reduce the pressure drag as much as Goldschmied claimed. Future efforts should be put forth optimizing the aft body to reduce the low pressure in front of the slot and improving aft entrance of the slot to allow for a smoother flow. 


\section{ACKNOWLEDGMENTS}

I would like to thank everyone that was involved in the thesis process. First I would like to thank my parents for supporting me through every step and helping anytime I got stressed out or needed anything. I would like to thank Josh and Nicole for bringing me on to their Goldschmied project to do the CFD for their model. Next I would like to thank Dr. Marshall for taking me on as a grad student and guiding me through the process and Dr. McDonald for taking me in under his wing to help with anything he could. Finally I would like to thank my roommates, Adam and Brian, for giving me support and helping show the way, you guys have greatly improved my time here at Cal Poly. 


\section{TABLE OF CONTENTS}

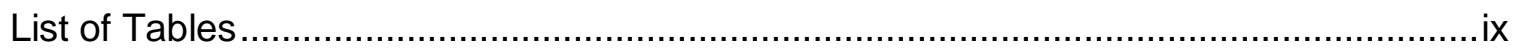

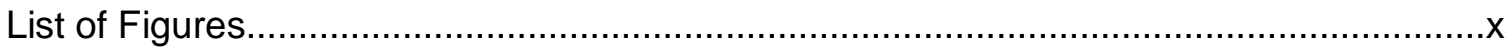

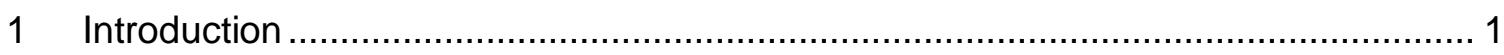

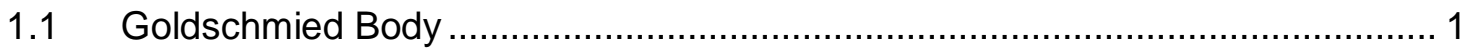

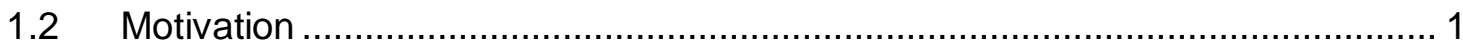

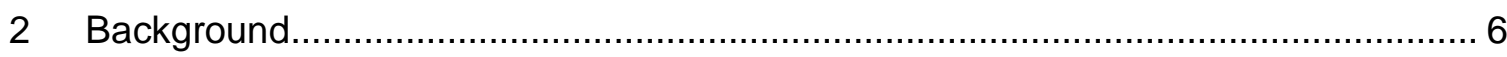

2.1 Boundary Layer Suction and Boundary Layer Ingestion ................................ 6

2.2 Early Wind Tunnel Testing ........................................................................... 7

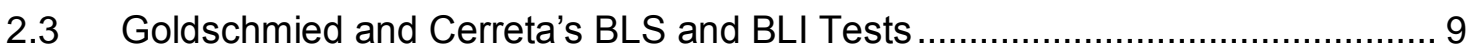

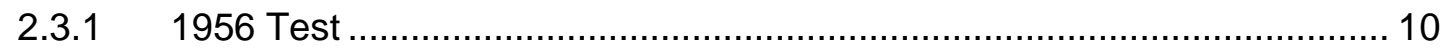

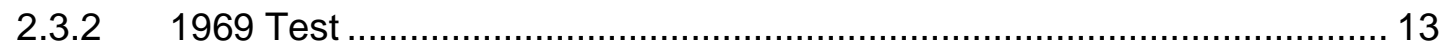

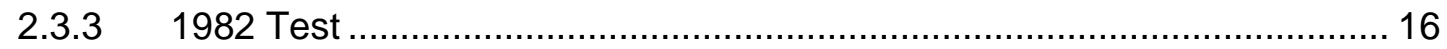

2.4 Recent Computational Fluid Dynamic Studies ............................................. 20

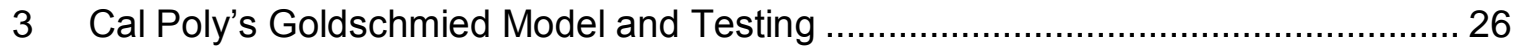

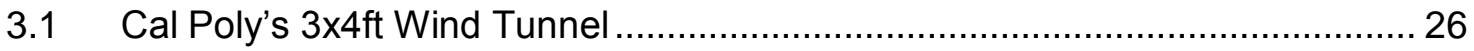

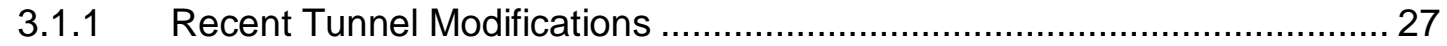

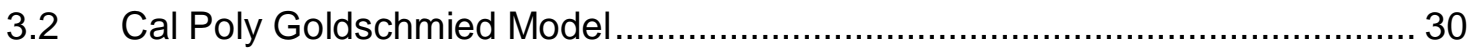

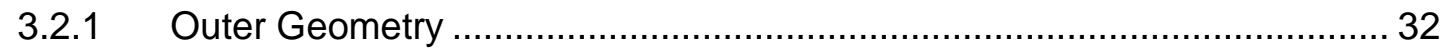

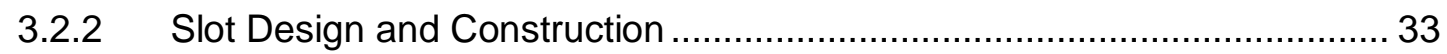

3.2.3 Tunnel Mounting Method .................................................................... 35

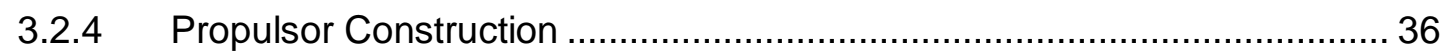

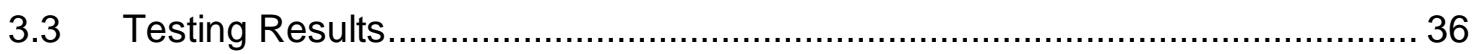

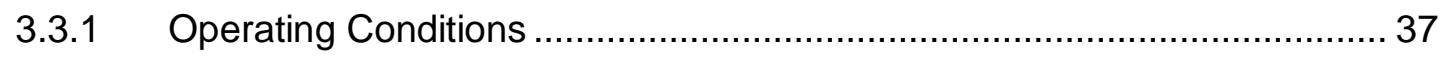

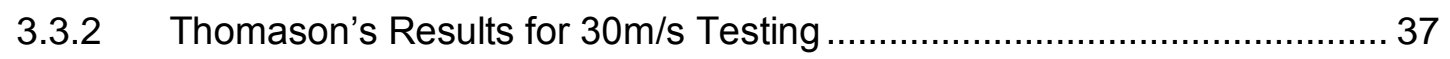

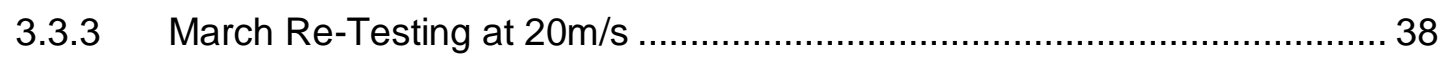

4 Computational Fluid Dynamics Approach ......................................................... 40

4.1 Model Component Simplifications ......................................................... 40

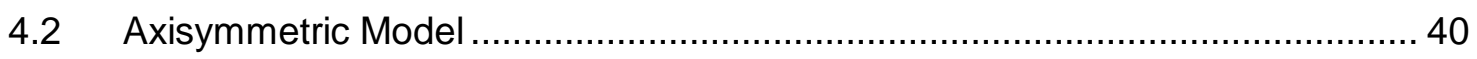

4.2.1 Axisymmetric Tunnel Simulation ............................................................. 41

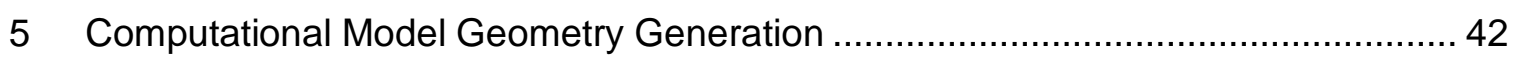

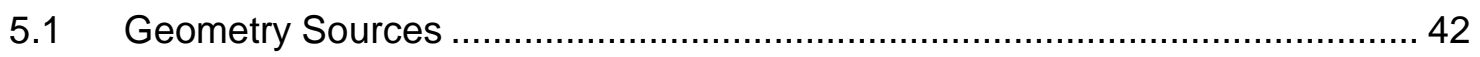

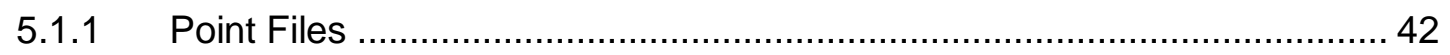




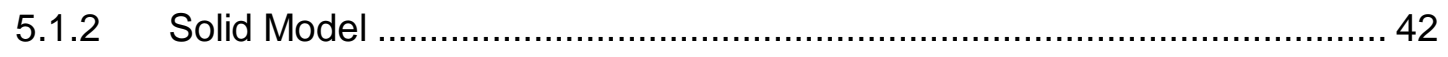

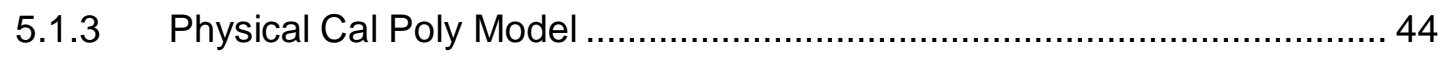

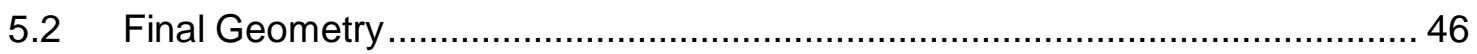

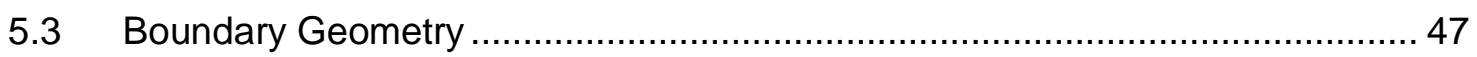

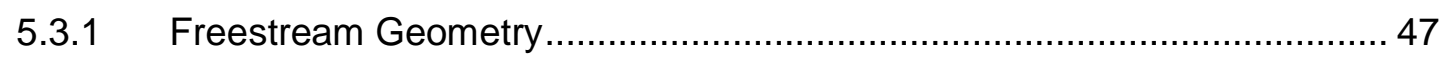

5.3.2 Straight Tunnel Geometry .............................................................. 48

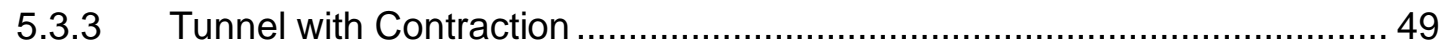

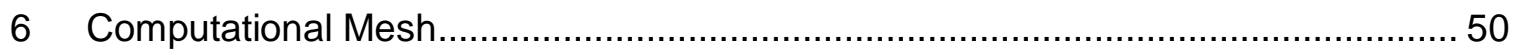

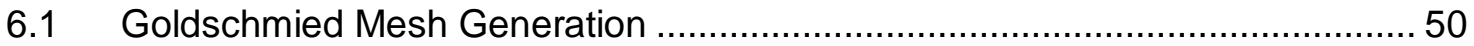

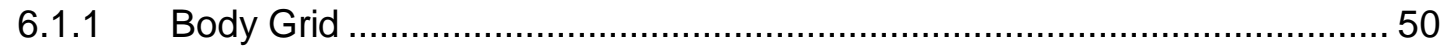

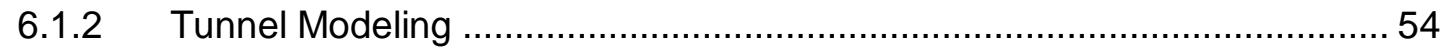

6.2 Surface Mesh and $Y+$ Calculation ……………….................................. 56

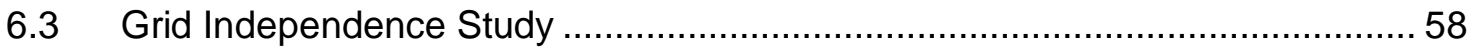

6.3.1 Grid Convergence Index (GCl) Method............................................... 58

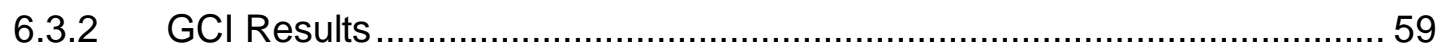

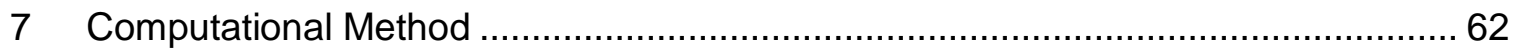

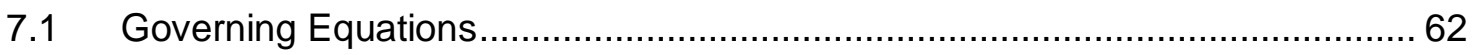

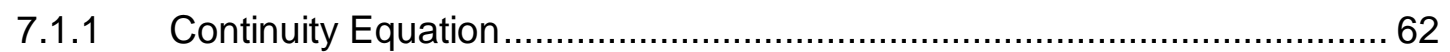

7.1.2 Momentum Equation......................................................................... 63

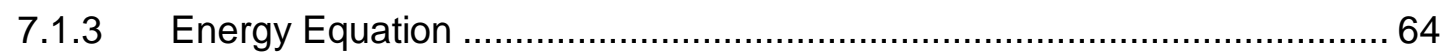

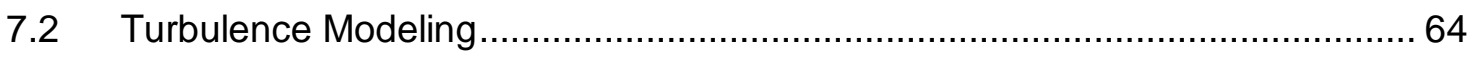

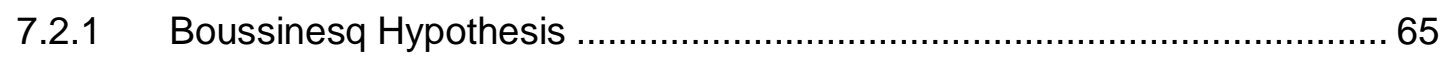

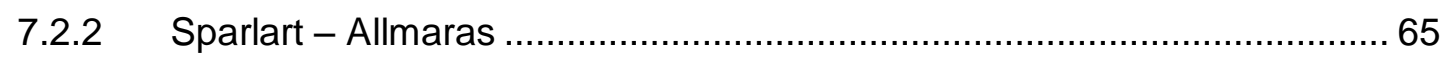

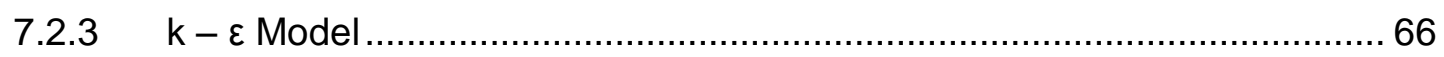

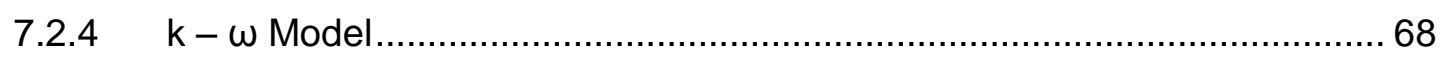

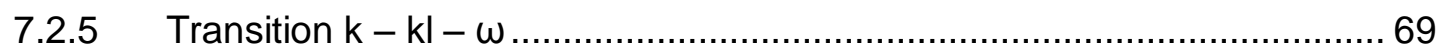

7.2.6 Transition Shear Stress Transport........................................................ 69

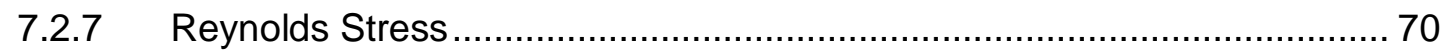

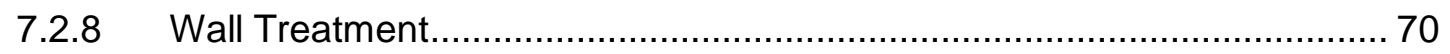

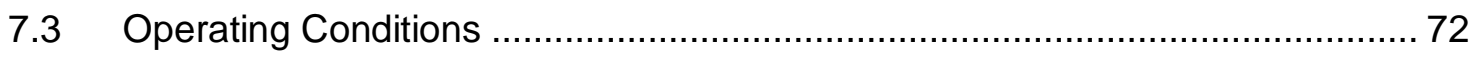

7.3.1 Boundary Conditions ………………………................................. 72

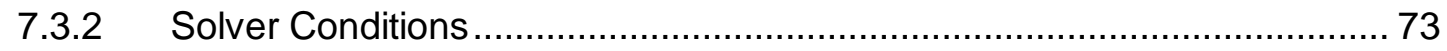

7.3.3 Convergence Criteria ....................................................................... 74

7.4 Computational Resources.................................................................. 75 


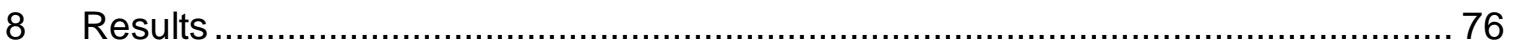

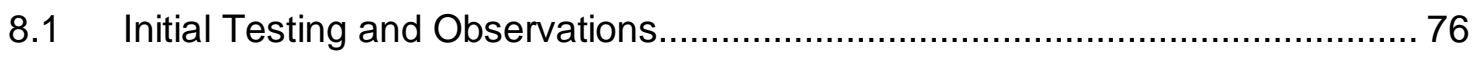

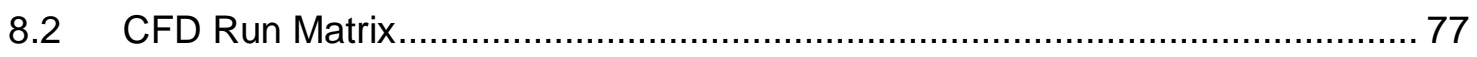

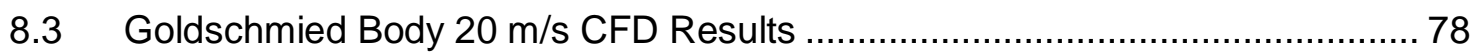

8.3.1 Initial Freestream Solution .............................................................. 78

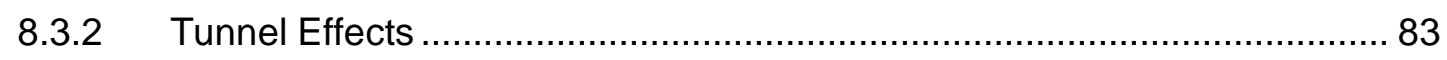

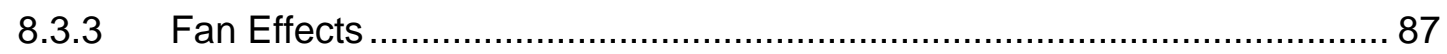

8.3.4 Turbulence Model Effects .................................................................. 96

9 CFD Comparison to Cal Poly's Tunnel Results .................................................... 98

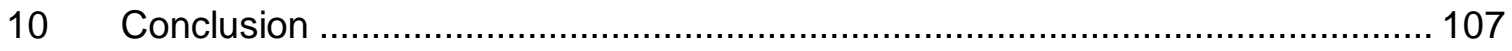

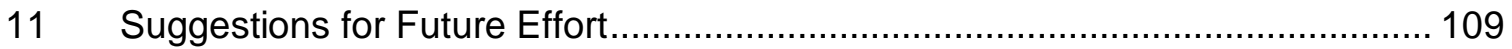

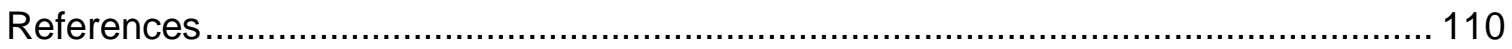

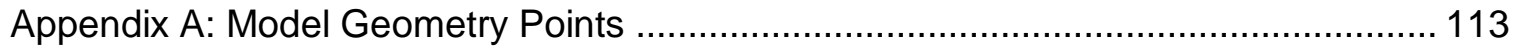




\section{List of Tables}

Table 1: Peraudo CFD results for the propelled BLC model ....................................... 25

Table 2. Summary of the original Goldschmied models compared to Cal Poly's

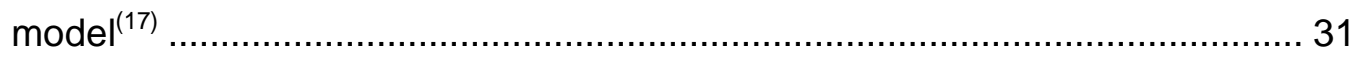

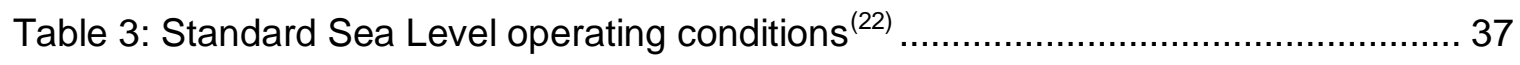

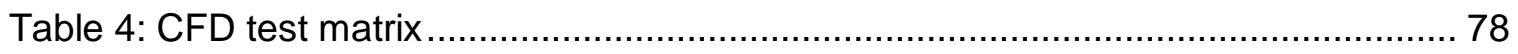

Table 5: Comparison of drag coefficients from free steam to wind tunnel conditions for both fan off and fan on at 500 pascals ................................. 86

Table 6: Pressure Drag differences due to turbulence models for the straight tunnel at different fan pressures ......................................................... 97 


\section{List of Figures}

Figure 1: Airframe technologies to reduce fuel burn for both an advanced Tube and Wing and Hybrid Body Aircraft ${ }^{(5)}$

Figure 2: Subsonic fuselage drag from skin friction and pressure drag as a function of fineness ratio ${ }^{(6)}$

Figure 3: Schematic layout of a 2-seat GA aircraft with integrated Goldschmied

Propulsor ${ }^{(2)}$ 4

Figure 4: Comparision of a conventional airship and a Goldschmied airship ${ }^{(7)}$.............. 4

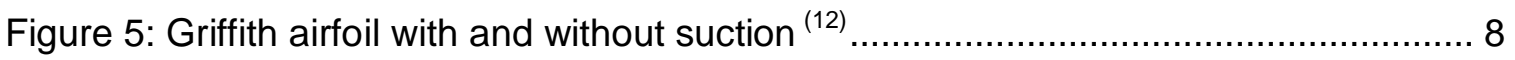

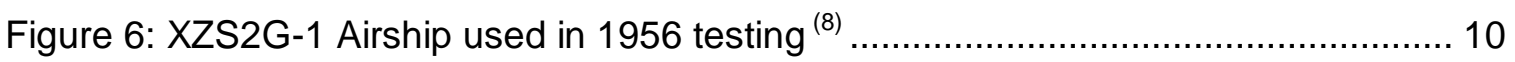

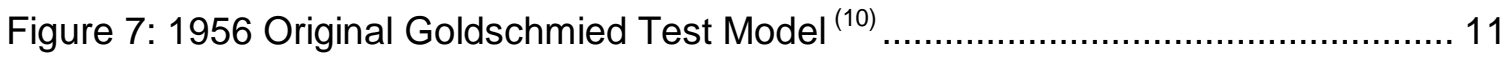

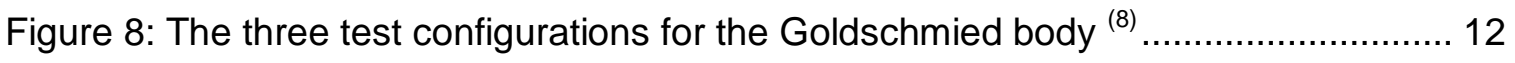

Figure 9: Clay filled cusp cross section added in the 1969 test ${ }^{(13)}$............................... 14

Figure 10: Ringleb scheme of a snow cornice used for cusp inspiration ${ }^{(14)}$.................... 14

Figure 11: Pressure distributions for $+/-6$ degree angle of attack for test model ${ }^{(13)} \ldots \ldots . .15$

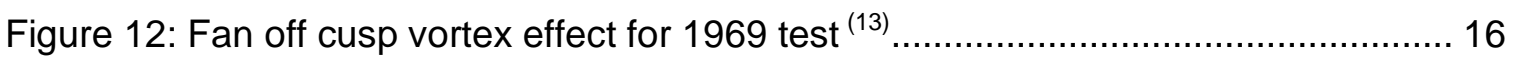

Figure 13: Internal schematic of the suction aftbody propulsor integration ${ }^{(9)} \ldots \ldots \ldots \ldots \ldots . . . . . .18$

Figure 14: Fabio Goldschmied with the 1986 model and attached tail section ${ }^{\left({ }^{9}\right)}$........... 19

Figure 15: Peraudo CFD velocity contours with k-omega SST turbulence model

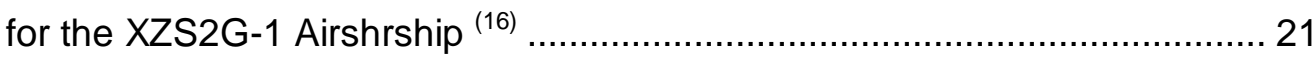

Figure 16: Medium mesh for the 1969 un-propelled Goldschmied model ${ }^{(16)}$................. 22

Figure 17: Medium structured mesh for the 1982 Goldschmied model ${ }^{(16)} \ldots \ldots \ldots \ldots \ldots \ldots . . . . . . . . . .23$

Figure 18: Velocity contour and streamlines for the propelled airship with attached flow ${ }^{(16)}$ 23

Figure 19: Peraudo CFD (a) and experimental (b) Cp Distributions for the self-

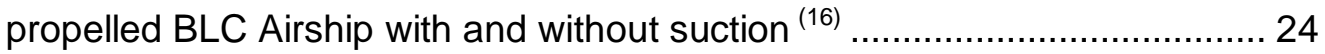

Figure 20: Top View of Cal Poly's 3x4ft Draw Down Wind Tunnel................................ 26

Figure 21: Wind tunnel inlet flow visualization before screen redesign ${ }^{(19)}$..................... 27

Figure 22: Wind tunnel inlet flow visualization after screen redesign ${ }^{(19)}$....................... 28

Figure 23: Velocity deviation in the improved Cal Poly wind tunnel course grid ${ }^{(20)}{ }^{\ldots . . . . . .2} 29$

Figure 24: Cal Poly wind tunnel course grid turbulent intensity plot ${ }^{(20)}$.......................... 29

Figure 25: Final Goldschmied SolidWorks Model .................................................... 32 
Figure 26: Comparison of body shapes used in Goldschmied's testing nondimensionalized by forebody length ${ }^{(7)(9)(10)(21)}$

Figure 27: Three cusp geometries that were constructed for the Goldschmied propulsor ${ }^{(18)}$ 34

Figure 28: Ducted fan propulsion unit and inlet rear face mounting 35

Figure 29: Flow visualization with Cusp $C$ and fan at full power showing separation 38

Figure 30: Experimental results from the March $20 \mathrm{~m} / \mathrm{s}$ re-testing. 39

Figure 31: SolidWorks model used to model the internal slot geometry 43

Figure 32: Discrepancies between the solid model connection ring and cusp and the final wind tunnel model 44

Figure 33: Pressure port locations on the aft body 45

Figure 34: Lip on the aft section of the wind tunnel model 46

Figure 35: Final slot geometry with cusp $A$ and aft lip modeled 46

Figure 36: Final body geometry with Cusp A and 0.385 " slot gap. 47

Figure 37: Freestream geometry 48

Figure 38: Straight tunnel geometry 48

Figure 39: Tunnel with contraction geometry 49

Figure 40: C-mesh around the freestream body geometry 51

Figure 41: $\mathrm{H}$-mesh around the tunnel body geometry 51

Figure 42: $\mathrm{H}$-grid showing the nose corner meshing 52

Figure 43: Aft section with slot grid meshing. 53

Figure 44: A close view of slot inlet meshing technique 54

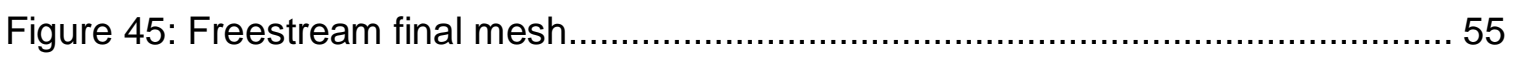

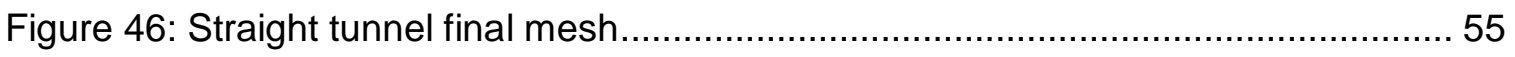

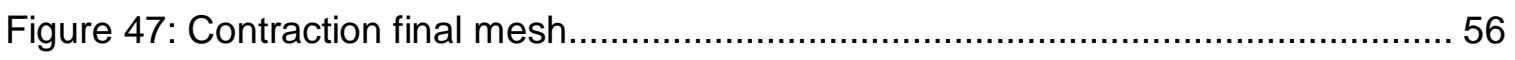

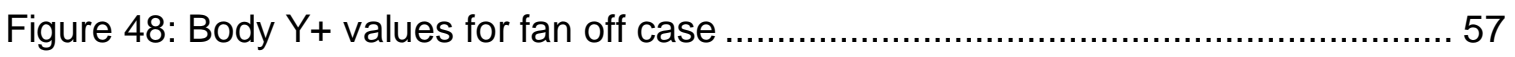

Figure 49: Body $\mathrm{Y}+$ values for fan on with a $\Delta \mathrm{P}$ of 1500 Pascals ............................... 58

Figure 50: Grid indepence for fan off conditions using the $\mathrm{GCl}$ method .......................6 60

Figure 51: Grid indepence check for fan on (500 pa) conditions using the $\mathrm{GCl}$

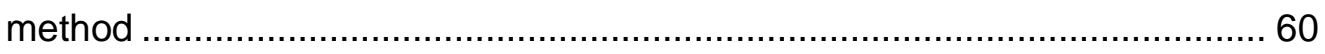

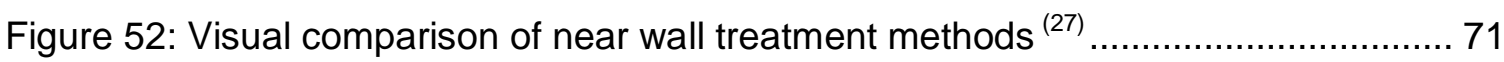

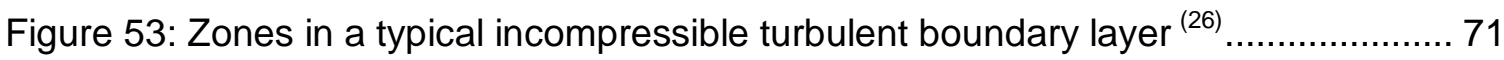


Figure 54: Contours of static pressure across the fan with a pressure increase of

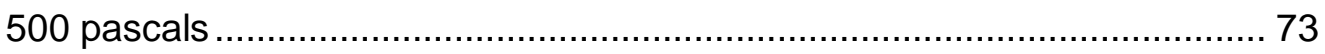

Figure 55: Velocity contours $(\mathrm{m} / \mathrm{s})$ for fan off and fan on at 500 pascals ..................... 79

Figure 56: Pressure contours (pascals) for fan off and fan on at 500 pascals............... 80

Figure 57: Streamlines colored by velocity $(\mathrm{m} / \mathrm{s})$ for fan off and fan on at 500 pascals

Figure 58: Close up rear streamlines colored by velocity $(\mathrm{m} / \mathrm{s})$ for fan off and fan on at 500 pascals 82

Figure 59: Inlet streamlines colored by velocity $(\mathrm{m} / \mathrm{s})$ for fan on at 500 pascals 83

Figure 60: Velocity contours $(\mathrm{m} / \mathrm{s})$ for straight tunnel geometry 84

Figure 61: Velocity contours $(\mathrm{m} / \mathrm{s})$ for tunnel contraction geometry 84

Figure 62: Tunnel effects on body $\mathrm{Cp}$ for fan off conditions 85

Figure 63: Tunnel effects on body $\mathrm{Cp}$ for fan on at 500 pascals . 85

Figure 64: Straight tunnel wall $\mathrm{Cp}$ 87

Figure 65: $\mathrm{Cp}$ disribution for fan pressures of 0 to 1500 Pascals 89

Figure 66: $C p$ vs. $R^{2}$ values for varying fan pressures. 90

Figure 67: CFD Body axial force build-up for different fan speeds. 92

Figure 68: Breakup of body sections between the fore, aft, and slot sectons 92

Figure 69: CFD Total force build-up for different fan speeds. 93

Figure 70: Velocity vector plots showing the flow at the inlet entrance for varying fan speeds.

Figure 71: Pressure drag coefficents for different turbulence models at different fan pressures 96

Figure 72: Comparison of Cp CFD data to Cal Poly experimental data for fan off conditions 98

Figure 73: Comparison of Cp CFD data to Cal Poly experimental data for the fan at $300 \mathrm{~Pa}$ 99

Figure 74: 3x4ft 3-D tunnel cross section static pressure contours for straight model at $20 \%$ chord. 101

Figure 75: 3x4ft 3-D tunnel cross section static pressure contours with model in 2 degrees of beta at $20 \%$ chord 102

Figure 76: Correlation between Cp difference at $20 \%$ chord and side slip angle (beta) 
Figure 77: Comparison of $\mathrm{Cp} \mathrm{CFD}$ data to Cal Poly experimental data for all fan conditions

Figure 78: Comparison of CFD fan speeds to Cal Poly experimental data: Aft Section 105

Figure 79: Experimental axial force for different geometries at the 5 different fan settings 106

Figure 80: Flood filled velocity contours for max fan speed. 108 


\section{Nomenclature}

\begin{tabular}{|c|c|}
\hline$A$ & Area $\left(\mathrm{m}_{2}\right)$ \\
\hline BLC & Boundary Layer Control \\
\hline $\mathrm{BLI}$ & Boundary Layer Ingestion \\
\hline BLS & Boundary Layer Suction \\
\hline$C_{D}$ & Total axial force Coefficient, $\frac{D}{q * V^{2 / 3}}$ \\
\hline $\mathrm{C}_{\mathrm{d}, \mathrm{p}}$ & Pressure Drag Coefficient, $\frac{D_{p}}{q * V^{2 / 3}}$ \\
\hline$C_{d, w}$ & Wake Drag Coefficient, $\frac{D_{w}}{q * V^{2 / 3}}$ \\
\hline Cp & Pressure Coefficient, $\frac{p-p_{\infty}}{q}$ \\
\hline $\mathrm{C}_{\mathrm{q}}$ & Suction Flow Coefficient \\
\hline $\mathrm{D}$ & Total axial force $(\mathrm{N})$ \\
\hline$D_{p}$ & Pressure Drag (N) \\
\hline $\mathrm{F}_{\mathrm{S}}$ & Factor of Safety \\
\hline $\mathrm{L}$ & Model length (m) \\
\hline$\dot{m}$ & Mass Flow Rate, $\rho A V(\mathrm{~kg} / \mathrm{s})$ \\
\hline$p$ & Static Pressure $(\mathrm{Pa})$ \\
\hline p & Apparent Order of Refinement \\
\hline$p_{\infty}$ & Freestream Static Pressure $(\mathrm{Pa})$ \\
\hline$P$ & Total Pressure $(\mathrm{Pa})$ \\
\hline Q & Volume Flow Rate $\left(\mathrm{m}^{3} / \mathrm{s}\right)$ \\
\hline$q$ & Freestream Dynamic Pressure, $\frac{1}{2} \rho V^{2}(\mathrm{~Pa})$ \\
\hline r & Radius $(\mathrm{m})$ \\
\hline $\mathbf{r}$ & Refinement Factor \\
\hline $\mathrm{Re}_{\mathrm{L}}$ & Reynolds Number, $\frac{\rho V_{0} L}{\mu}$ \\
\hline $\mathrm{T}$ & Temperature $(\mathrm{K})$ \\
\hline$u^{*}$ & Friction velocity $\sqrt{\frac{\tau_{w}}{\rho}}$ \\
\hline V & Velocity $(\mathrm{m} / \mathrm{s})$ \\
\hline
\end{tabular}




$\begin{array}{ll}\vec{v} & \text { Freestream Velocity Vector } \\ & \text { for 3D: } \vec{v}=\left(\mathrm{v}_{\mathrm{x}}, \mathrm{v}_{\mathrm{y},}, \mathrm{v}_{\mathrm{z}}\right) \text { or } 2 \mathrm{D} \text { axisymmetric: } \vec{v}=\left(\mathrm{v}_{\mathrm{x}}, \mathrm{v}_{\mathrm{r}}\right) \\ V_{B} & \text { Model body volume }\left(\mathrm{m}^{3}\right) \\ \mathrm{y}^{+} & \text {Dimensionless wall distance } \frac{u^{*} y}{v} \\ \mathrm{x} & \text { Distance from nose }(\mathrm{m}) \\ \mathrm{x}, \mathrm{r} & \text { 2-D Asymmetrical Coordinates } \\ \mathrm{x}, \mathrm{y}, \mathrm{z} & \text { 3-D Cartesian Coordinates } \\ \mathrm{Greek} \text { Characters } \\ \rho & \text { Density } \\ \vec{\tau} & \text { Stress Tensor } \\ \tau_{w} & \text { Wall shear stress } C_{f} * 1 / 2 \rho U_{\infty}^{2} \\ \mu & \text { Dynamic Viscosity } \\ v & \text { Kinematic Viscosity } \mu / \rho\end{array}$




\section{Introduction}

\subsection{Goldschmied Body}

The motivation behind the recent Goldschmied propulsor research is to provide knowledge on the reduction of blunt body drag by a method that combines boundary layer suction and boundary layer ingestion for the propulsor. In the 1950's through the 1980's Fabio Goldschmied looked these self-propelled bodies like airships and underwater vehicles and from his research he found a means to greatly reduce drag.

\section{"As compared to wind-tunnel tests of conventional streamlined bodies at exactly the same volume Reynolds number, the integrated vehicle design requires $\sim 50 \%$ less power for both free-transition and tripped transition cases"}

\section{-Fabio Goldschmied describing his results from testing ${ }^{(1)}$}

The vehicle uses a boundary layer suction system through a single suction slot on the aft section to increase pressure recovery. This slow moving air is then fed into the inlet of the propulsion system, which provides a more efficient propulsive system than a conventional system that takes in free stream velocity. Goldschmied is credited at combining these two technologies in a synergistic method that has the potential to greatly decrease the power needed to move a blunt body through the air or water.

\subsection{Motivation}

If this idea is applied to an aircraft fuselage ${ }^{(2)}$, while the overall drag reduction would not be as large for an airship, there would still be a great reduction in drag. Fuselage drag for most subsonic general aviation aircraft accounts for 30 to $50 \%$ of the 
total zero drag of an airplane..$^{(3)}$ If that drag can be reduce by $50 \%$, there would be an overall vehicle drag reduction of $15-25 \%$. This would be a huge finding, as current commercial wing and tube designs typically find single percent point decreases in drag as a great increase in drag reduction. Groups including NASA and Boeing, have placed much more importance on drag reduction as the current growth of the airline industry is projected to more than double in the next 20 years $^{(4)}$ in addition to the cost of fuel. These groups have set metrics to drastically reduce noise and emissions along with increasing commercial vehicle performance. These metrics include 10, 15 and 20 year goals, with the 2020 mark being a 50\% decrease in aircraft fuel/energy consumption as compared to 2005 best in class aircraft. ${ }^{(5)}$ NASA has also included a projected breakdown of where they predict these improvements will come from. This can be seen below in Figure 1.

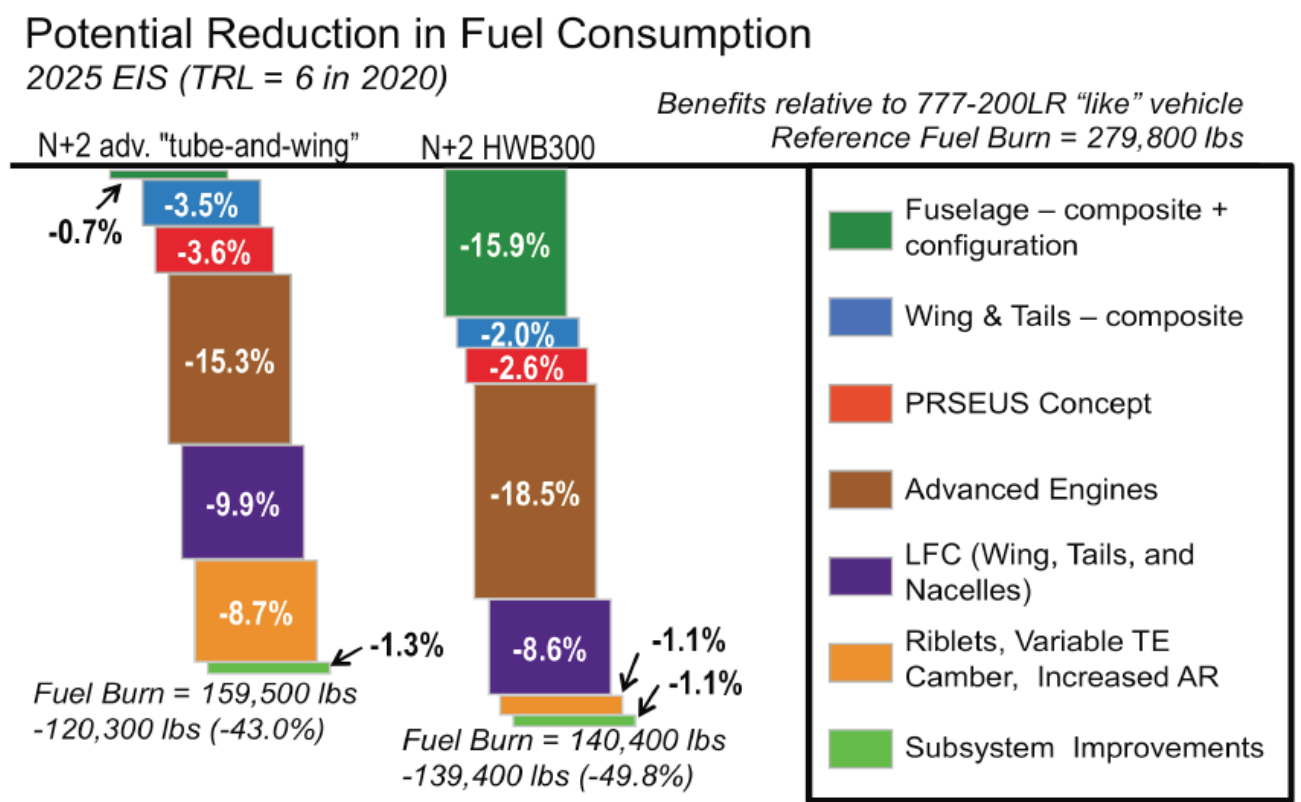

Figure 1: Airframe technologies to reduce fuel burn for both an advanced Tube and Wing and Hybrid Body Aircraft ${ }^{(5)}$

This shows that for a standard wing and tube aircraft, only a $0.7 \%$ decrease in fuel is expected. With a drastic vehicle reconfiguration, like a blended body aircraft, a potential $15.9 \%$ decrease in fuel usage is expected. As stated earlier, Goldschmied 
projected a 15 to $25 \%$ decrease in vehicle drag. This was due to the elimination of pressure drag. When looking at Figure 2 for a standard subsonic transport, the lowest base drag occurs at a fineness ratio of approximately $1 / 3$ where the coefficients of friction and pressure drag are about equal. If the pressure drag was eliminated, we would see roughly a $50 \%$ decrease in drag $^{(6)}$, similar to what Goldschmied claimed.

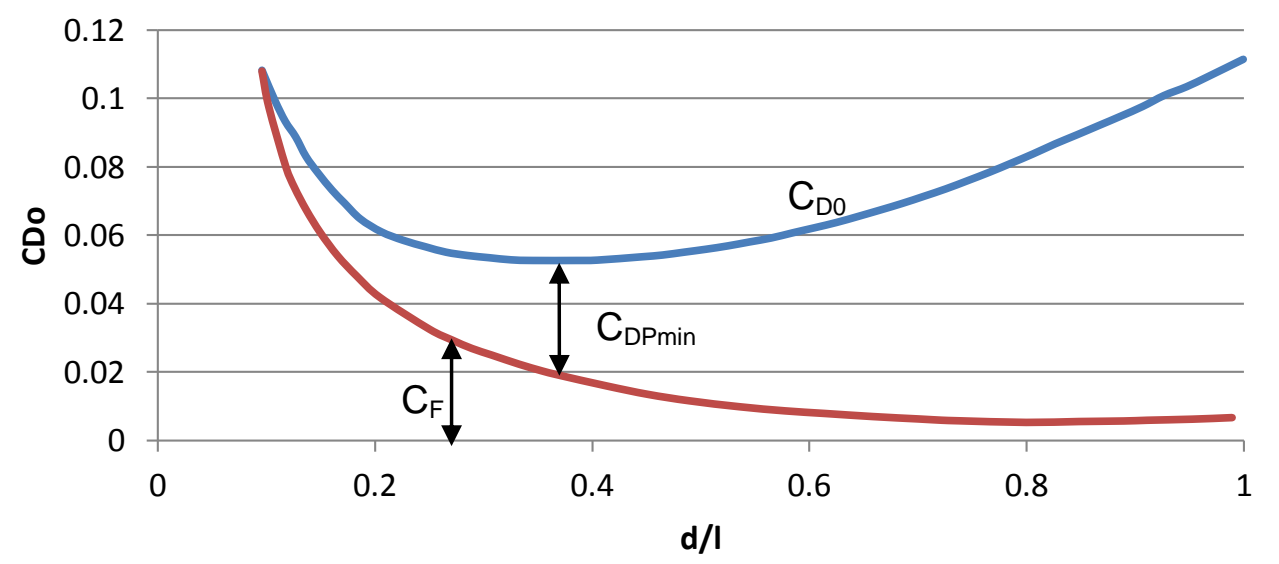

Figure 2: Subsonic fuselage drag from skin friction and pressure drag as a function of fineness ratio $^{(6)}$

If this drag reduction could be obtained, it will have a huge impact on the aviation industry. Two examples of this can be seen in Figure 3 and Figure 4 on the next page. 


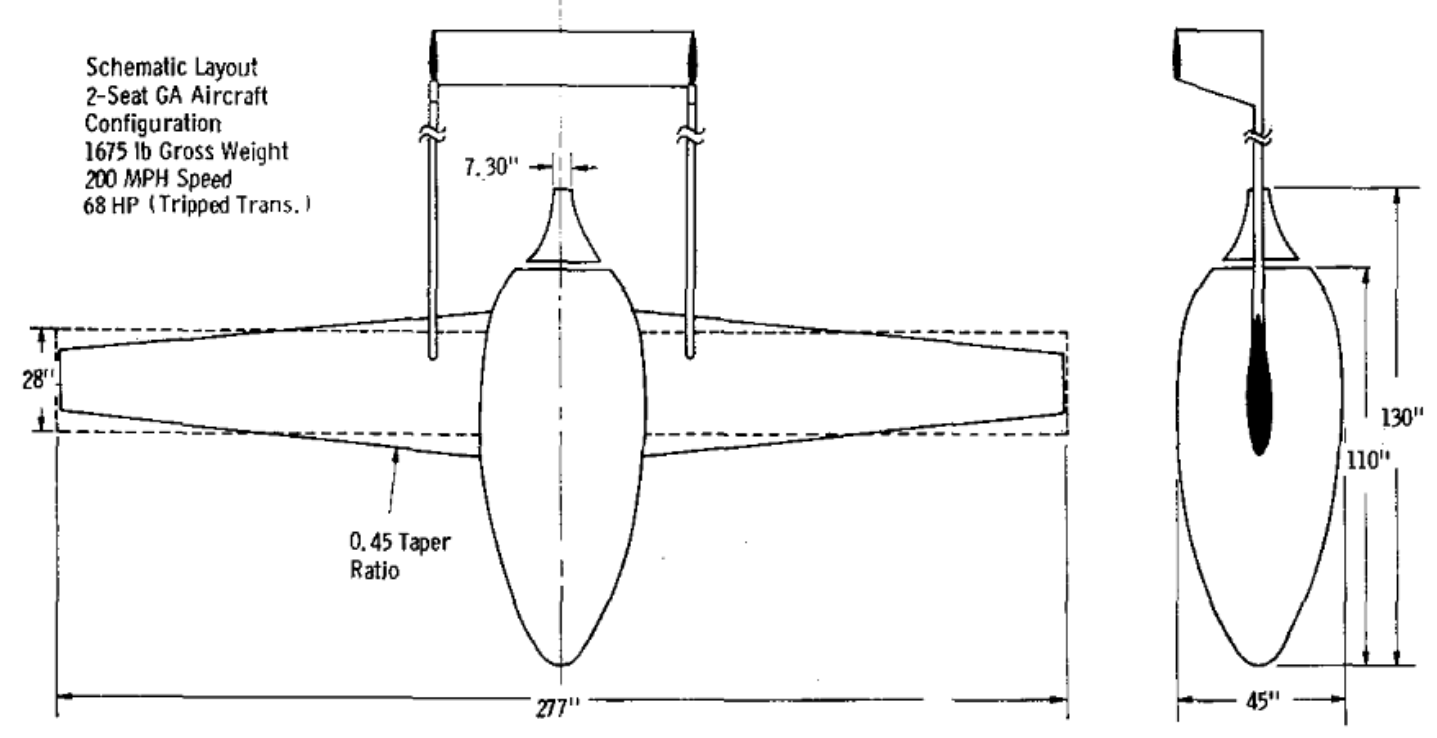

Figure 3: Schematic layout of a 2-seat GA aircraft with integrated Goldschmied Propulsor ${ }^{(2)}$

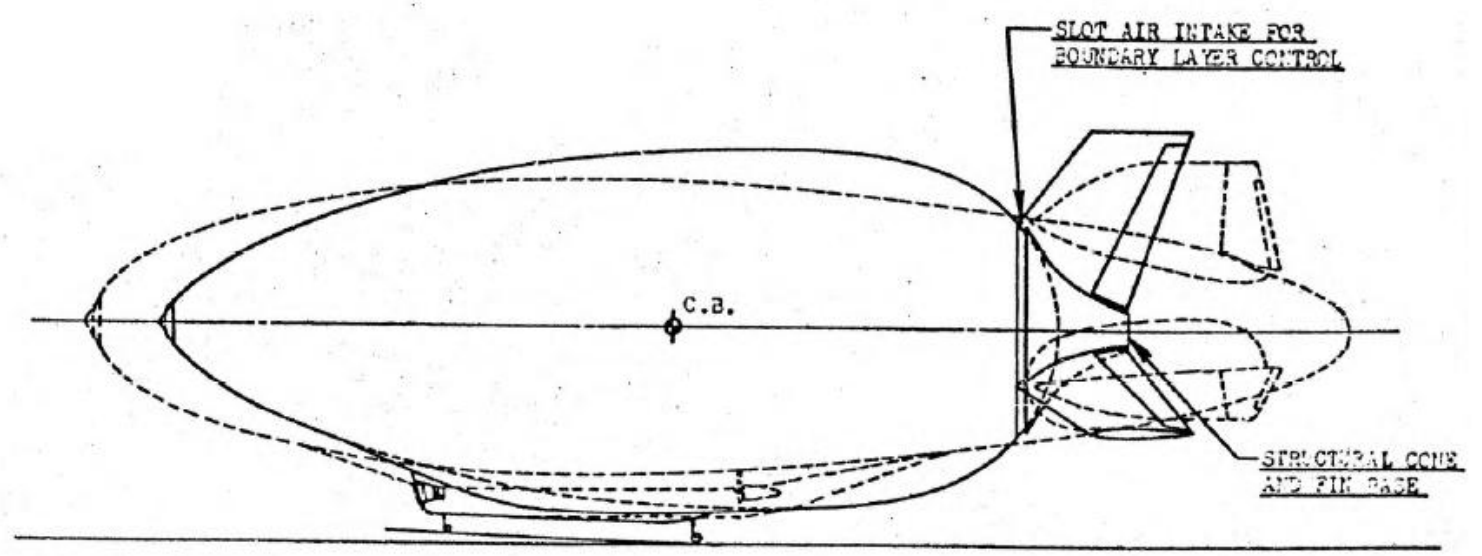

Figure 4: Comparision of a conventional airship and a Goldschmied airship ${ }^{(7)}$ 
This is very enticing, however, there is little information beside the handful of wind tunnel tests that Goldschmied and Cerreta have conducted to support this finding. ${ }^{(1)}$ (7)(8)(9)(10) A lot of their test information either left questions about the geometry, procedures, or was just not available. To evaluate Goldschmied's claims, two graduate students at California Polytechnic State University in San Luis Obispo have created a wind tunnel model and computational fluid dynamics model to try to replicate the earlier wind tunnel results. 


\section{Background}

The idea of boundary layer control, both with suction and blowing, is nothing new. The technology has been around since the 1920 's ${ }^{(11)}$ with some of the earliest wind tunnel testing being completed in the early 1940's. ${ }^{(12)}$ The research done has been promising but it has yet to 'buy' itself onto a commercial vehicle. Goldschmied aimed to fix this with multiple wind tunnel tests and wrote papers on the possible integration with general aviation aircraft. This idea has yet to catch on, due partially to the mechanisms involved, but also due to the lack of knowledge and data in the subject. The background presented here is to show what has been done and let us pick up where Goldschmied and others have left off, with the goal of getting a better understanding of the synergy of boundary layer suction and ingestion.

\subsection{Boundary Layer Suction and Boundary Layer Ingestion}

There are two main technologies that are used on the Goldschmied Propulsor: boundary layer suction and boundary layer ingestion. While both can offer improvements independently of each other the real advantage for the Goldschmied Propulsor is that it uses both technologies at once to obtain a synergistic result to give the greatest efficiencies.

Boundary layer suction (BLS) works by removing the slow moving boundary layer which allow for higher energy flow to come down to the surface. The BLS method results in a thinner boundary layer that can stay attached longer. This lack of separation due to BLS is advantageous in aircraft applications because it allows for higher lift airfoils, as the onset of stall is extended. It also can reduce pressure drag, as the newly attached flow can withstand a higher pressure recovery than the slower moving flow that was removed. 
Boundary later ingestion (BLI) works by ingesting the boundary layer for use in the propulsor unit. With a BLI system, the flow is already going relatively slow upon entering the propulsor and greater propulsive efficiency is possible because the system requires less energy to get the same increase in momentum from the low speed flow as it would for a higher speed flow. Ideally, if a system is able to ingest all the slow moving air around a vehicle it will just need to speed up the flow back to freestream velocity to counteract all drag.

The Goldschmied system uses both of these technologies to create a system that greatly reduces the pressure drag while increasing the propulsor efficiency in one single mechanism.

\subsection{Early Wind Tunnel Testing}

The earliest test of boundary layer ingestion for the reduction of pressure drag was seen in wind tunnel testing as early as 1944 in the National Physical Laboratory in London, England by E. Richards and W. Walker ${ }^{(12)}$. The test involved a $16 \%$ thick Griffin airfoil that spanned the width of the $9 \mathrm{ft}$ tunnel with a $4 \mathrm{ft}$ center section that was isolated using wing plates. The airfoil had a $6 \mathrm{ft}$ chord and was run at a Reynolds number of $25 \times 10^{6}$. The center section housed a suction slot, pressure ports, and all of the instrumentation. This was done to investigate the two dimensional flow and the behavior of the slot. Although they showed that they had a better pressure recovery on the aft section, there was not much change from the power off condition because the flow stayed attached at all settings, including fan off. This is largely a result of the relatively thin airfoil and the smooth long aft section that keeps the flow attached. The pressure recovery change due to the suction can be seen in Figure 5 below. 


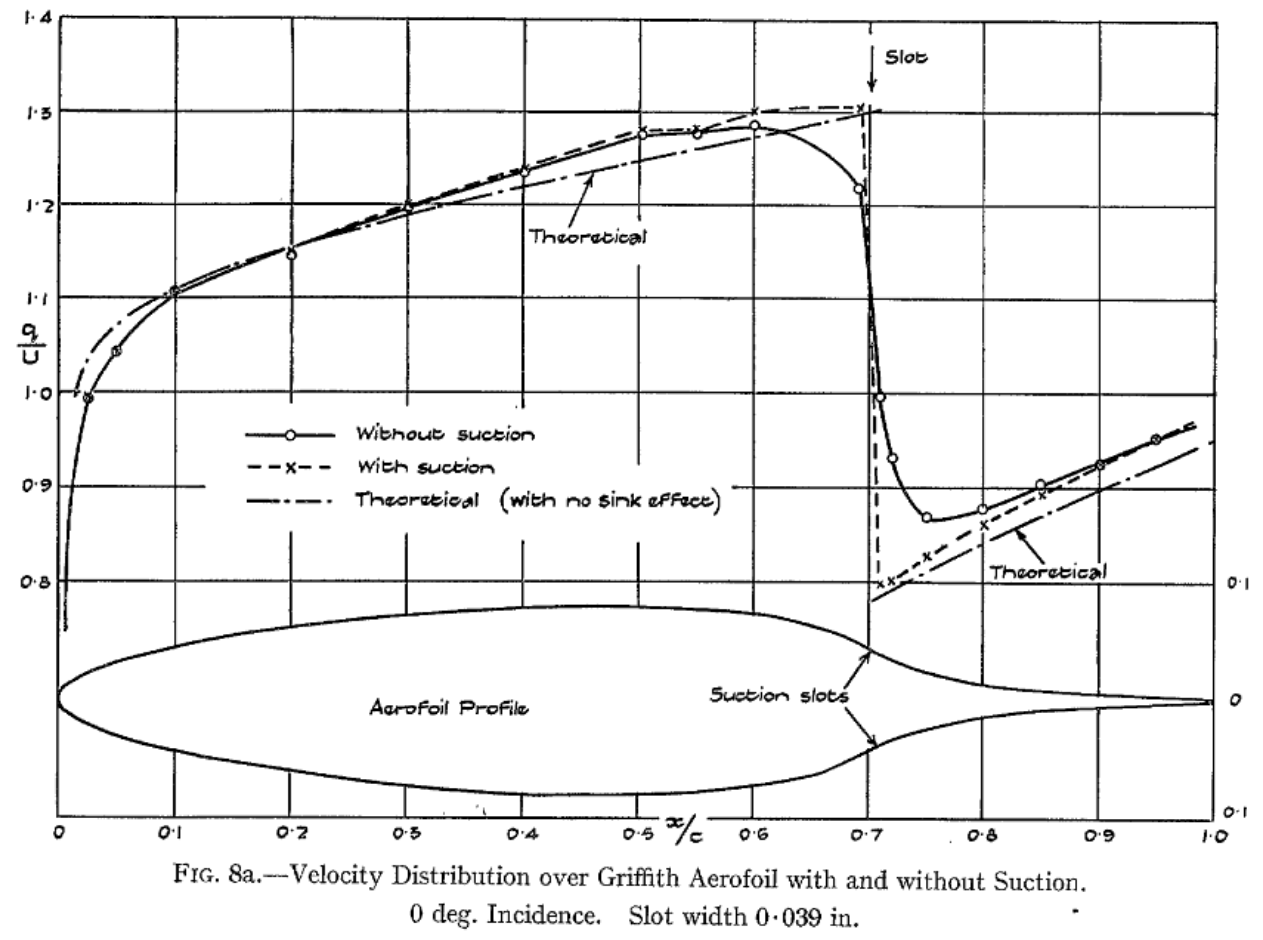

Figure 5: Griffith airfoil with and without suction ${ }^{(12)}$

Another item they were looking at was total drag reduction, which included looking into maintaining laminar flow for as long as possible. Initially they had many difficulties getting a smooth geometry. The initial body had a large amount of waviness that would trip the boundary layer and make it turbulent. They noticed that small changes could have a large effect on the overall behavior of the flow. Their testing also included trying to get the boundary layer to stay laminar across suction slots and continue on the aft section. They were able to do this for a small section but because of the concavity of the aft region along with the adverse pressure gradient they were ultimately unsuccessful. At the end of the paper they provided ten points that summarized what they found. ${ }^{(12)}$ The most helpful points can be seen below:

(1) Backward-facing slots seem to be slightly more efficient than forward-facing slots 
(2) Slot widths up to three time boundary layer thicknesses may be safely used, but the proportion of the boundary layer absorbed increases with increase of slot width

(3) A slot width at least equal to the laminar boundary layer thickness at the slot should be used to prevent high frictional losses at the duct entry.

(5) With transition at any point forward of the slot, between 0.05 and 0.10 of the turbulent boundary layer air at the slot must be absorbed to prevent separation (as indicated by silk threads)

(8) For slot widths greater than that of a single laminar boundary layer thickness, the suction head with minimum suction is less for forward transition than with laminar flow to the slot.

(9) No improvement in effective drag coefficient can be obtained by causing transition forward of the slot. On the other hand, the effect of forward movements of transition will be no greater than for a normal low-drag wing.

With forward transition, the extra suction needed to establish the nonseparated flow regime over that needed simply to maintain it, is no greater than with laminar flow to the slot.

\subsection{Goldschmied and Cerreta's BLS and BLI Tests}

As stated earlier, Fabio Goldschmied spent a lot of time and effort trying to prove the gains from the propulsor. He was the main proponent of this technology and spent over 30 years looking at the concept. Because of this we look to him as an expert in that subject area, hoping to glean from his previous testing to help us move forward with ours. 


\subsubsection{Test}

The initial testing of the Goldschmied body was run at the Aerodynamic Laboratory at the David Taylor Model Basin. There are two main papers that documented this test, one by Cerreta $^{(8)}$ that was a report to U.S. Navy and a second done for Goodyear Aircraft Company ${ }^{(7)}$. This test was initially planned to span three months but this was greatly reduced because of budget concerns. This changed the overall test approach from finding what combination of aft shape, slot gap, fan speeds, and additional geometry that provided an optimal configuration to a test approach that demonstrated what initial designs showed promise for further research.

\subsubsection{1956 Wind Tunnel Test models}

Two models were tested: the first being a XZS2G-1 Airship which was modeled at a $1 / 70^{\text {th }}$ scale at $58.8^{\prime \prime}$ and the second being the Goldschmied model that was made from a modified Griffith/Lighthill airfoil to be the same volume as the XZS2G1. The XZS2G-1 airship was one used as a model because it was one of the lowest drag airships of its day, and would make a good reference point for drag comparisons. The geometry can be seen below in Figure 6.

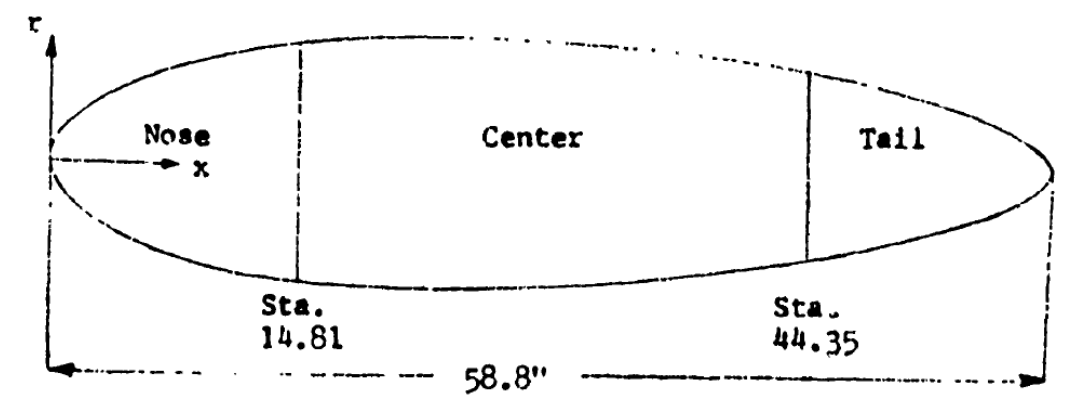

Figure 6: XZS2G-1 Airship used in 1956 testing $^{(8)}$

The Goldschmied body being tested was setup as a proof of concept model. The suction slot was fed by a pump that was located external to the wind tunnel. This allowed 
them to solely look at the BLI on the aft body and see how that compared to the airship model and not have to work on integrating a fan in the aft section. The geometry was constructed from a Lighthill/Griffin Airfoil. This geometry was chosen as the maximum thickness is farther back than most airfoils, which helps maintain laminar flow and small boundary layers. This model was not optimized but thought to be a good enough shape that would function as a technology demonstrator and if successful the body could later be optimized. The model geometry can be seen below in Figure 7. There were also three main aft configurations that were looked at. The first configuration was considered the 'standard' with a straight slot and a concave aft section. The second configuration change added a shroud around the aft section facing forward to help the flow enter the slot. The third configuration was an annular ring that was mounted above the aft body. The annular ring had the slot closed and was tested to see if it could replicate the effect of the slot. All three configurations can be seen in Figure 8.

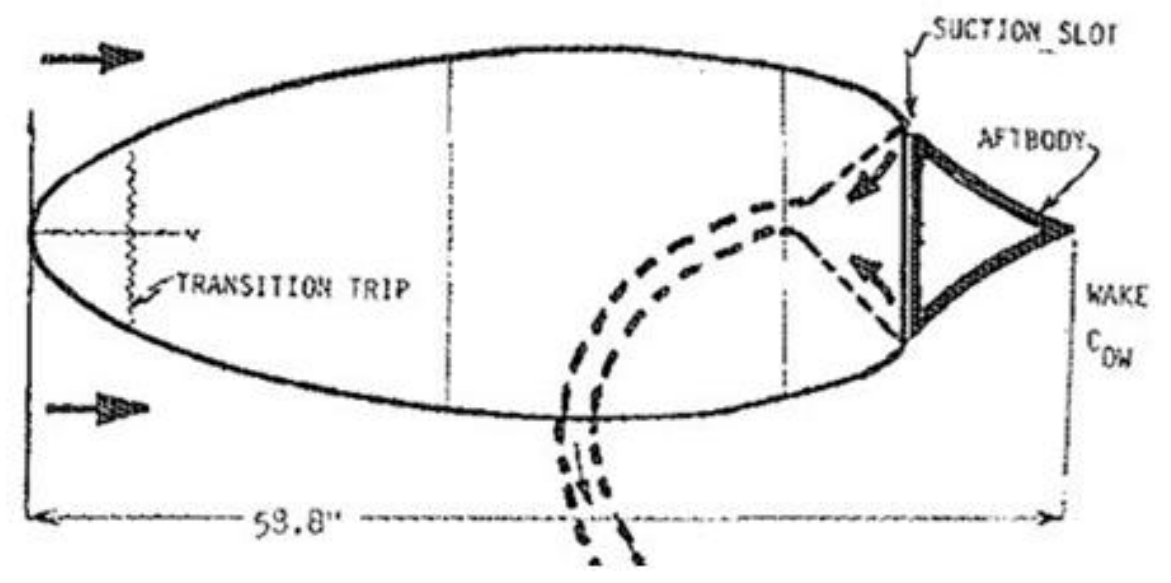

Figure 7: 1956 Original Goldschmied Test Model ${ }^{(10)}$ 


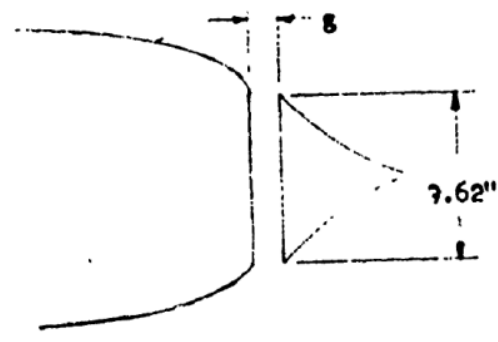

(a) Iatl cone configuration

$8 / 2=0.004,0.008$,

$0.012,0.016$

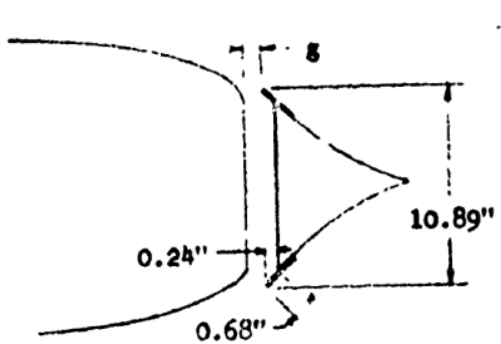

(b) Ta1l cone with Shroud

$g / L=0.004,0.008,0.012$

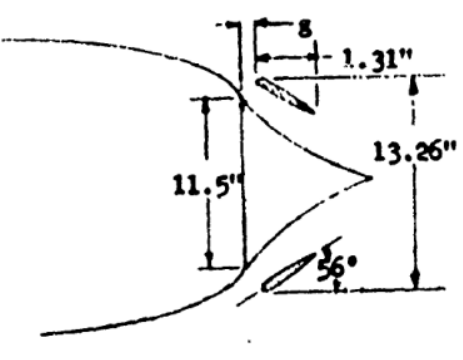

(c) Mnoular A1rfoll Configuration

$\varepsilon=0.007,0.013$,

Figure 8: The three test configurations for the Goldschmied body ${ }^{(8)}$

\subsubsection{1956 Test Results}

Since Goldschmied \& Cerreta had limited time in the wind tunnel, they only found general trends and results, mainly setting up a starting point for future tests. From the initial XZS2G-1 airship, Goldschmied \& Cerreta saw that the Goldschmied body with fan off had about $50 \%$ more drag. Then when the suction was turned on, the drag was greatly reduced, going to a fraction of the initial. This drag value was added to an estimated 'suction drag' that was computed to estimate the power needed by the suction, to get the total drag. This total drag was calculated $20-30 \%$ less drag than the XZS2G-1 airship. Another important factor discovered is that once the suction rate was increased enough to attach the flow on the aft section, any increase after that had little to no effect on the body's drag. Also, the tail cone with the shroud slightly decreased the power needed for flow attachment but no other effects after that. For the final configuration with the annular airfoil, they found that this did not decrease the drag at all but rather it increased it worse than the initial fan off condition without the annular airfoil. From this Goldschmied \& Cerreta concluded that the main conclusion was that the suction kept the flow attached and reduced drag by more than $20 \%$. 


\subsubsection{Test}

The second test of the Goldshmied body was done in 1969. During this test Goldschmied \& Cerreta resurrected the initial 1956 model and added more measurement instruments along with an added cusp at the slot entrance. The main paper for this test could not be located. The only information for this test was found in later papers that referenced this $2^{\text {nd }}$ test and reproduced only a small fraction of the original plots and numbers. For reference, the original test paper was cited in a later

report $^{(1)}$ as: "Aerodynamic analysis of the 1969 wind-tunnel test of the Goldschmied body Vol.I -Slot geometries and body distribution; Vol. II Boundary-layer suction, transition and wake drag," Westinghouse Electric Corp., R\&D Center, Research Report 77-1E9BLCON (March 1977). The most comprehensive information was reproduced in 1978 in Goldschmied's body optimization paper ${ }^{(13)}$, which is our main source of knowledge concerning the 1969 test.

\subsubsection{Modified Geometry}

One of the main improvements during this test was the addition of a cusp at the slot entrance. This cusp supposedly both decreased drag at the power off condition and increased suction stability while decreasing needed fan power to keep the flow attached. The best image of this cusp is seen in Figure 9. There are no coordinate points or information about the exact geometry. 


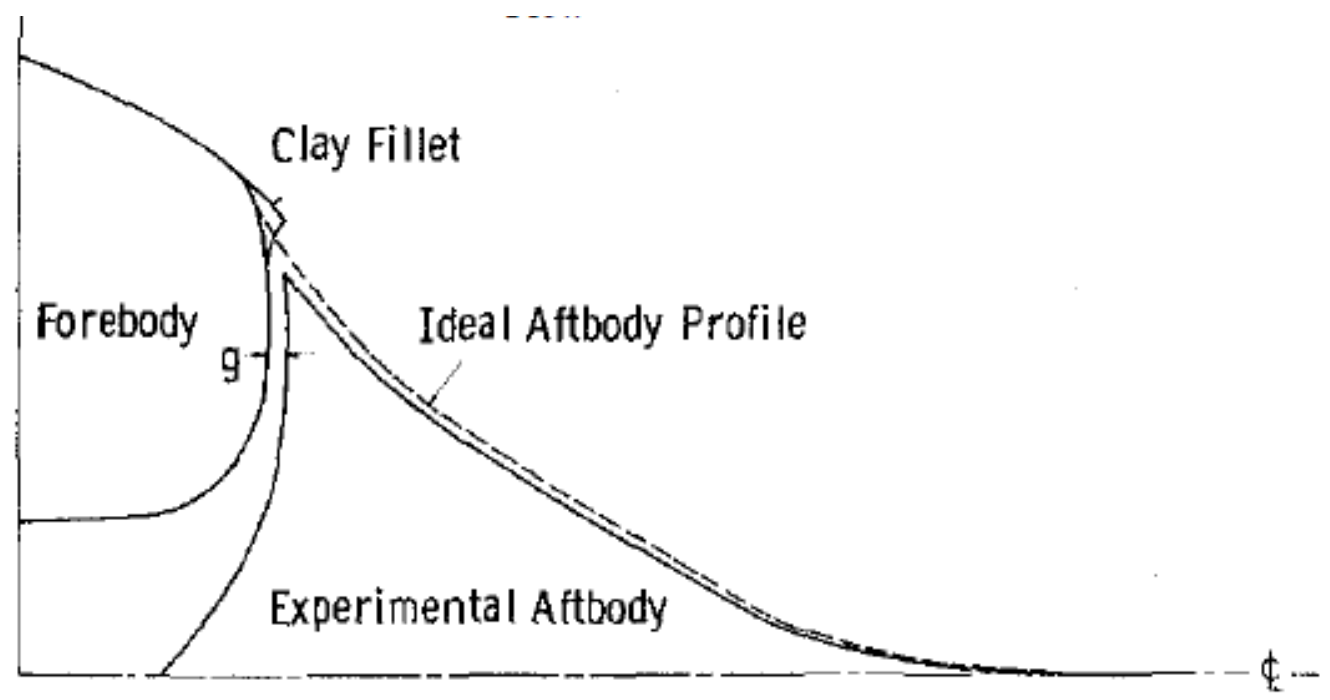

Figure 9: Clay filled cusp cross section added in the 1969 test $^{(13)}$

The paper does reference that the geometry was inspired by a paper by Friedrich Ringleb in 1961. ${ }^{(14)}$ Ringleb talks about the natural formation of ice cusps on ridges due to the air and snow circulating. As small quantities of ice get deposited, the cusp slowly forms into a shape that helps the flow circulate around the corner. This design is supposed to be better because, unlike a solid surface, the flow at the separating streamline is moving, which removes a lot of the shear stresses that take energy out of the flow. The inspiration for the cusps can be seen in Figure 10 below.

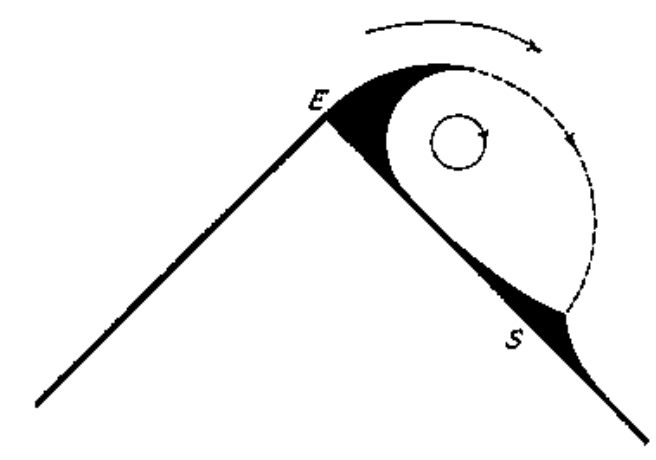

Figure 10: Ringleb scheme of a snow cornice used for cusp inspiration ${ }^{(14)}$

From the report it seems that this cusp greatly improved the performance, but there is very little information on the actual geometry. 
Finally the model was tested with $a \pm 6^{\circ}$ angle of attack. These tests were done to see how the Cp changed and if the aft section was adversely affected.

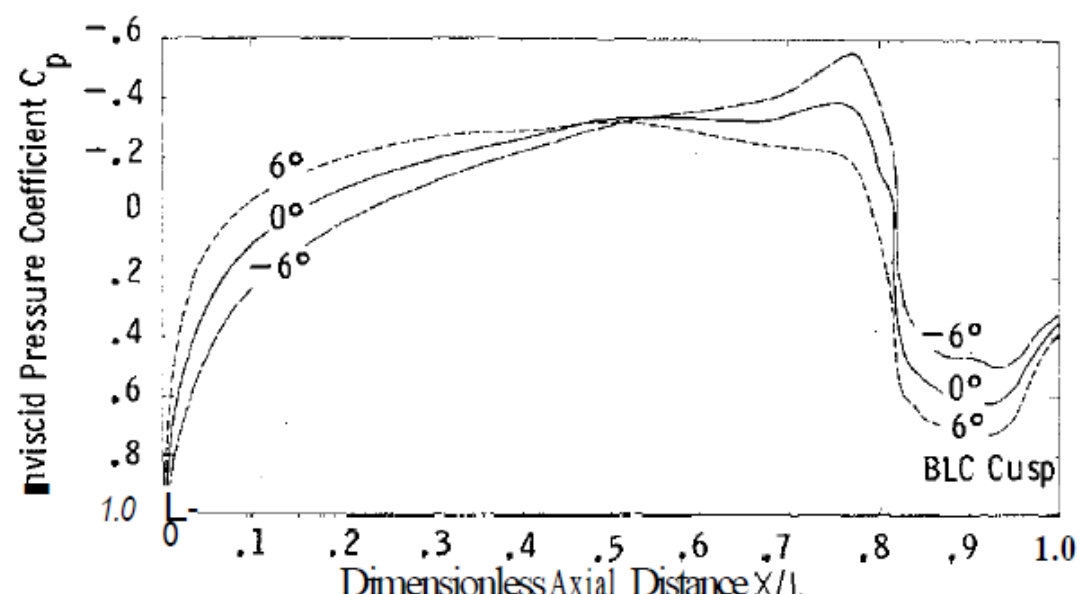

Figure 11: Pressure distributions for $+/-6$ degree angle of attack for test model ${ }^{(13)}$

From the graph in Figure 11 we can see that the six degree angle change did not result in drastic changes to the flow around the body. It behaved as expected, with the fore section increasing in pressure and the aft section decreasing in pressure at six degrees of inclination.

\subsubsection{Results of the 1969 Testing}

As stated earlier, the actual test document could not be found. Because of this we do not have Goldschmied's full data set or exact test conditions, rather we have a few graphs that were reproduced in later papers by Goldschmied, this can be seen in Figure 11 above and Figure 12 below. 


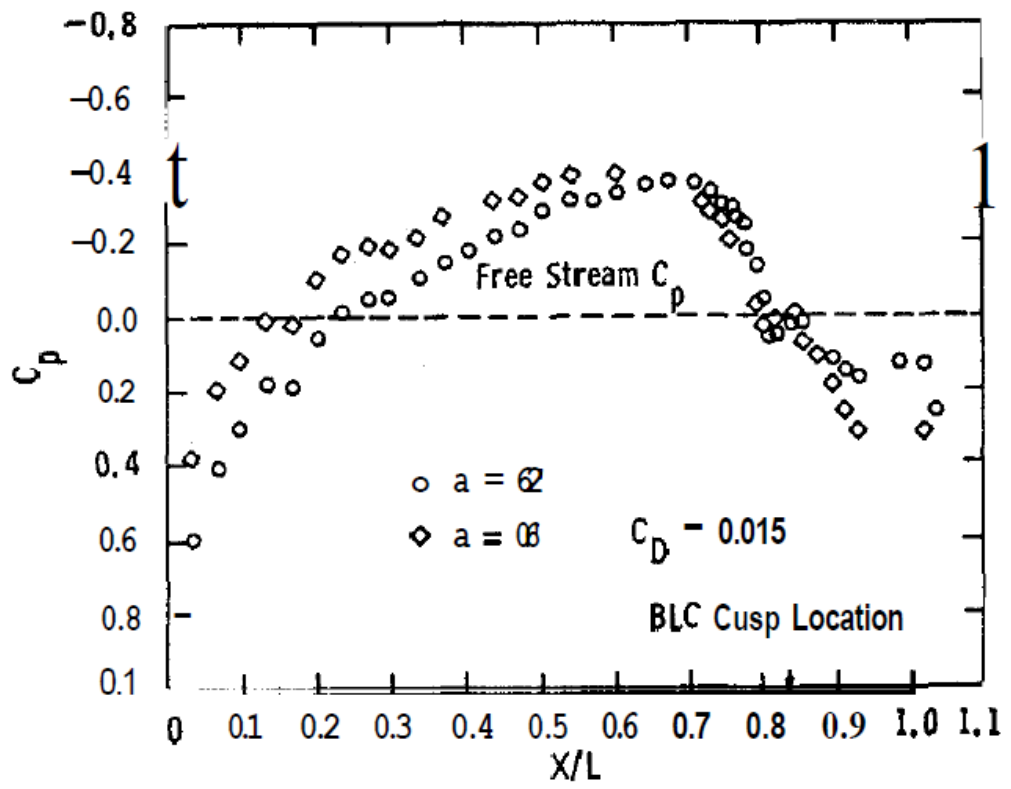

Figure 12: Fan off cusp vortex effect for 1969 test $^{(13)}$

From Figure 12 we see that they tested the fan off conditions and saw that the cusp provided passive suction that helped increase the pressure recovery on the aft section, increasing it to nearly a Cp of 0.35 . There isn't much more on this besides a sentence or two and leaves a lot to be desired by the reader. The statement with the most useful summary of the 1969 test was mentioned in Goldschmied's body optimization paper ${ }^{(13)}$. In this, he states: "The best configuration yielded a drag coefficient of $C_{D}=0.0144$ at the volume Reynolds number of $R=3.13 \times 10^{6}$; laminar boundarylayer flow was maintained up to $\sim 70 \%$ body length by actual wind-tunnel China Clay visualization."

\subsubsection{Test}

In 1982, Goldschmied completed his third and final test on the Goldschmied body. This test was aimed at integrating a propulsion unit into the aft section of the body. This had not been done previously, as an external pump had been used for the suction slot in the last two tests. The 1982 test used the same wind tunnel model as before but 
modified the aft section for the acceptance of a propulsion unit, referred to as a "Suction Aftbody Propulsor" or SAP.

\subsubsection{1982 Model Condition}

The Goldschmied model was not in that great of condition, as it was originally built in the mid 1950's and was not taken care of beyond a slight refurbishment in 1969 . The test condition of the model was described by Goldschmied:

"The aluminum forebody was rather eroded and corroded by its 25 years life and was far from the polished gleaming surface of "laminar" bodies; it was simply cleaned and tested without addition of transition trips (roughness strips or wires) for Confs. 00 and 01. (free transition cases)."(15)

There were also recollections that in addition to the erosion and corrosion, the model was "also dented"(1) from storage and previous handling.

\subsubsection{1982 Model Modifications}

The Goldschmied wind tunnel model was modified in the 1982 test to represent a statically stable and self-propelled body, something that was thought to be an example of fully integrated vehicle option. To do this, two main items had to be added to the model: a propulsor that needed to ingest the boundary layer and expel out the back while being $100 \%$ internal to the vehicle, and a rear empennage that would add stability.

The suction aftbody propulsor took up a large section of the internal volume, as seen in Figure 13 below. The SAP was a two stage fan that initially did not perform as needed. Flow straighteners were added to help with the performance. Even with this addition the peak efficiency was only $52 \%$, running at 11,700 RPM with 700 CFM flow and an exit gauge pressure of 9.5 inches of water. To get to the fan, the flow had to enter from the slot, change direction and head to the front of the model, where it turned 
around again to enter the fan inlet. This two $180^{\circ}$ vane-less corners leading up to the fan were noted to be inefficient and should be improved in future models.

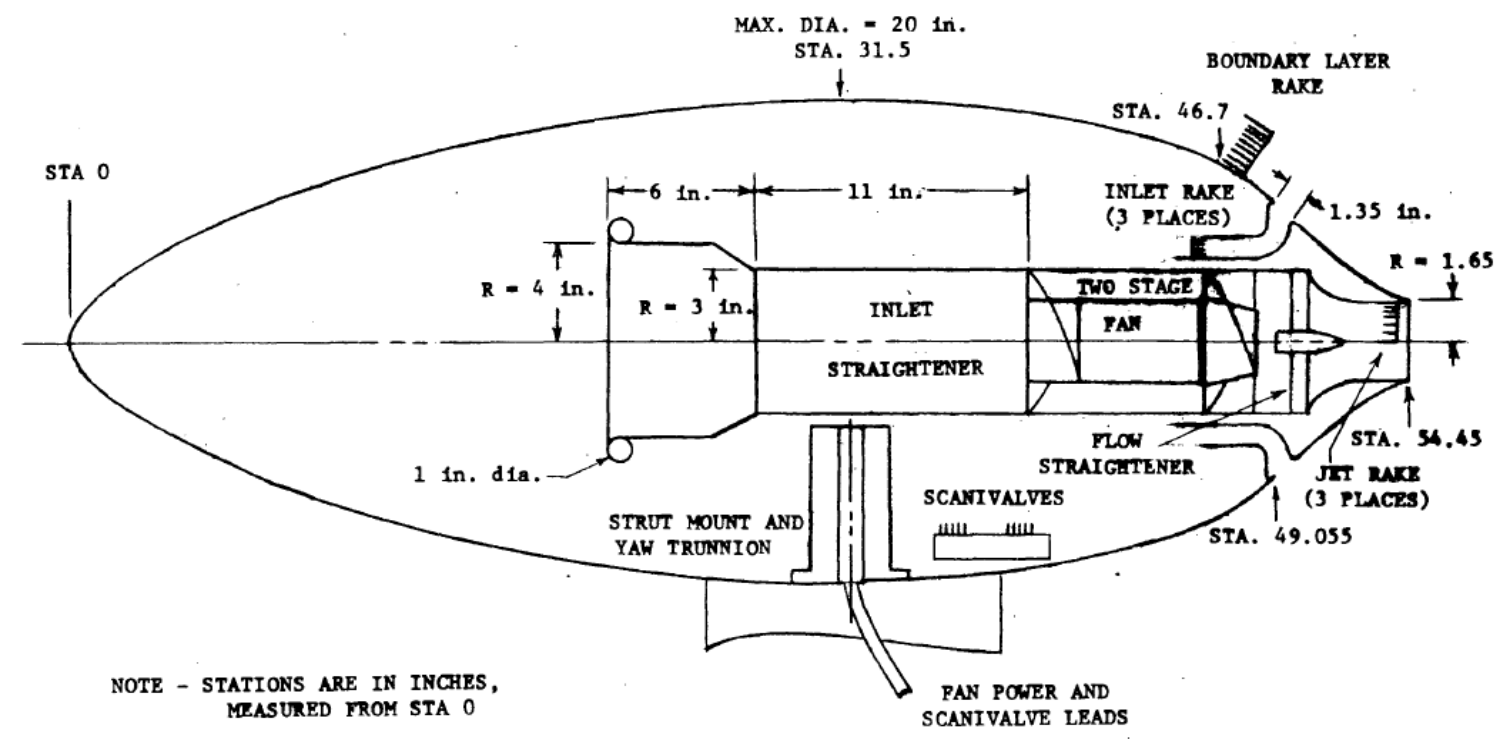

Figure 13: Internal schematic of the suction aftbody propulsor integration ${ }^{(9)}$

Finally an empennage was added that extended out of the center of the exit slot. The empennage extended out half the length of the original and was no larger than the maximum diameter of the forebody. The exact geometry details were catalogued in the 1982 test report by Howe and Neumann ${ }^{(9)}$. The final model with empennage can be seen in Figure 14. 


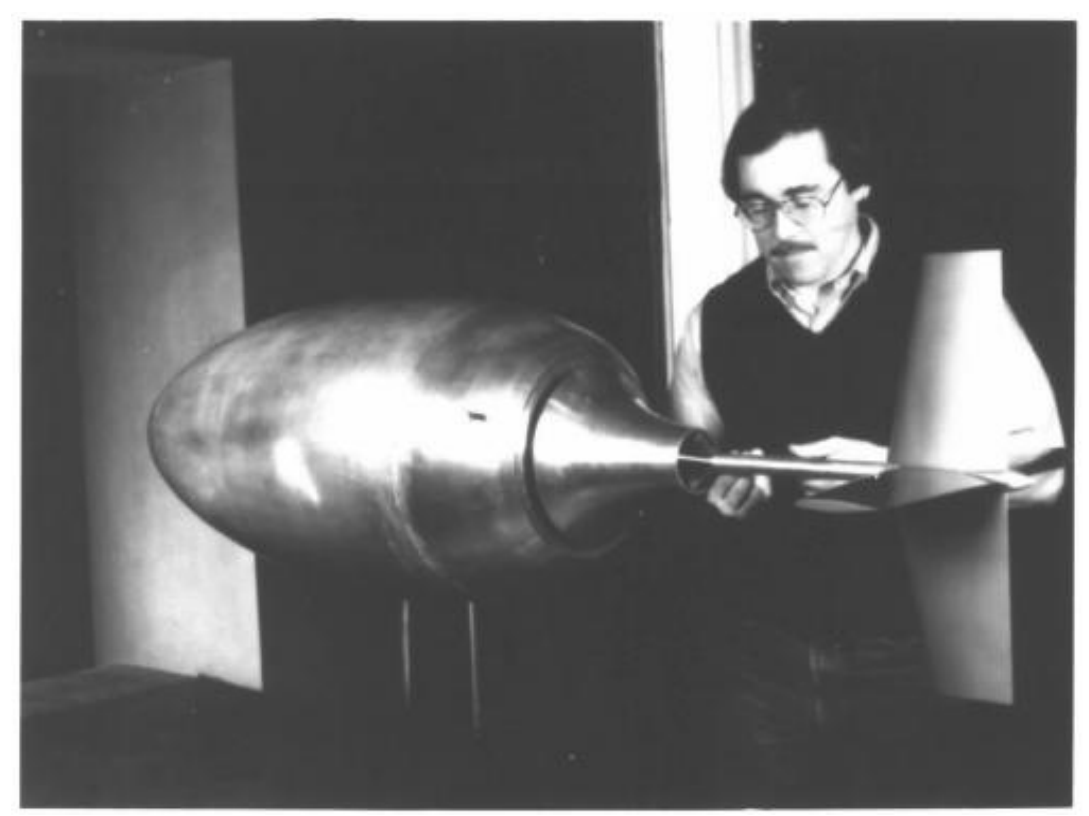

Figure 14: Fabio Goldschmied with the 1986 model and attached tail section ${ }^{(9)}$

\subsubsection{1982 Testing Results}

When collecting results, there were two methods used to calculate drag, one from the sting and the second from a wake survey. These results were far from ideal, as the sting was not able to accurately tare with the interference of the strut/model. The inability to accurately tare the values causes the validity of the sting forces to be questioned. For the wake survey, there were no azimuth measurements conducted so they were not totally sure the readings were in the center of the wake. Additionally there were large variations in the turbulent wake measurements, which bring up questions about the "steady state" values.

Even with these hardships Goldschmied claims that the tests saw $~ 50 \%$ drag reduction compared to that of a conventional streamlined body and that the added empennage added the stability needed for the model to be statically stable. These results were very similar to the results from the previous two wind tunnel tests. 
Goldschmied concluded from this testing that three elements must be incorporated in future designs to get the best efficiencies and boundary layer stabilities. The first being a suction slot, to ingest the boundary layer. The second element is a cusp on the inlet of the suction slot, as seen from the 1969 test. And finally a tail boom is needed to help stabilize the flow exiting the fan.

\subsection{Recent Computational Fluid Dynamic Studies}

Up until recently there has been no CFD replication or validation of the Goldschmied body. The paper "Computational Study of the Embedded Engine Static Pressure Thrust Propulsion System" by Peraudo et al. ${ }^{(16)}$ looked to provide the CFD basis for an embedded propulsion system. They did this by replicating Cerreta and Goldschmied's tests as described above. All CFD cases were run in FLUENT with structured grids of approximately one million cells. All geometries used the Grid Convergence Index $(\mathrm{GCl})$ to estimate uncertainties due to discretization error. Initial runs looked at different turbulence models, but the k- $\omega$ SST model gave the best results, so from here out we will be quoting those numbers.

\subsubsection{ZXS2G-1 Airship}

The first test case Peraudo et al. ran was of the ZXS2G-1 Airship test done by Cerreta in $1957^{(8)}$. The geometry with CFD results of velocity and streamlines can be seen in Figure 15. Overall the CFD for the airship matched well, especially with the experimental wake profile. The drag was slightly under predicted by about $15 \%$ for this case, with a $C_{D, W}=0.0242$ compared to the experimental of $C_{D, W}=0.0284$. The author stated that because of coarse geometry information, the missing experimental data, and approximations in the experimental data reduction used in the 1957 test, that $15 \%$ differences in the solution was still in agreement overall. 


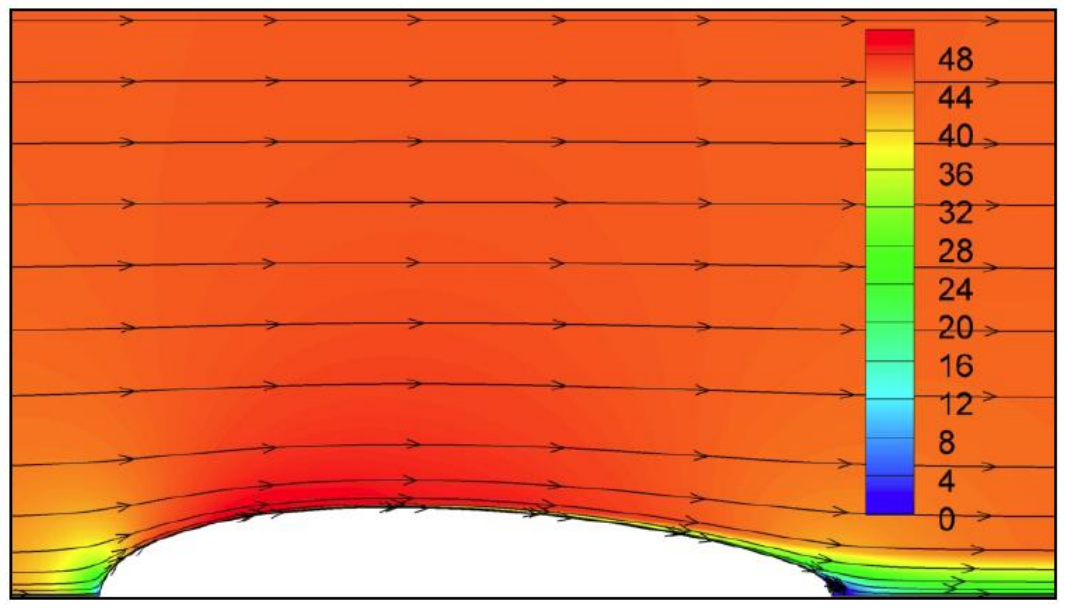

Figure 15: Peraudo CFD velocity contours with k-omega SST turbulence model for the XZS2G-1 Airshrship ${ }^{(16)}$

\subsubsection{1969 Test Model}

The next model that was tested was the initial Goldschmied body with boundary layer ingestion, as can be seen in Figure 16. Here two cases were looked at, one with suction off and one with suction on. The methods used were very similar to the first trial with the main difference being the difference between the CFD and experimental fan off drag values. The CFD gave a fan off drag value of $C_{D, w}=0.0307$, which was about a $20 \%$ increase from the ZXS2G-1 airship and the experimental value was given as $C_{D, W}=$ 0.0558. This seemed really high and was a lot different than the 1982 fan off test results of $C_{D, W}=0.0340$, so it was Peraudo's recommendation to disregard Goldschmied's high fan off drag values, as it seemed to be either a typo or anomaly. The fan on values are close to the experimental values, with CFD value of 0.0053 and the experimental value 0.0052 . 


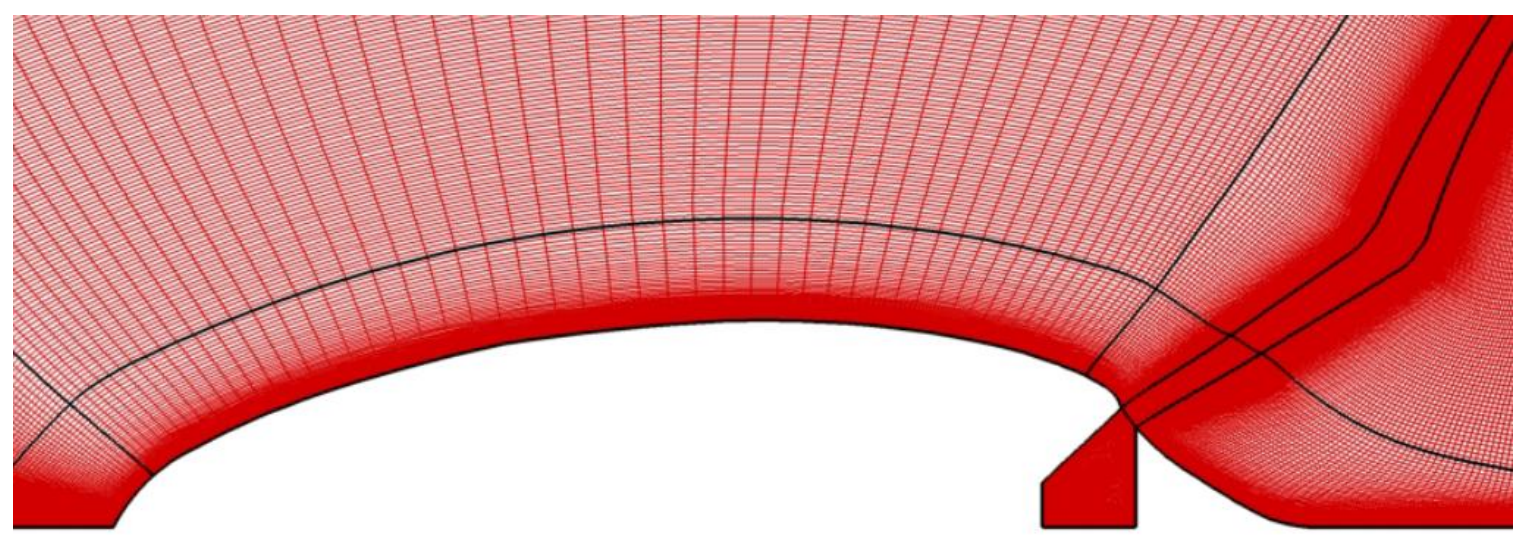

Figure 16: Medium mesh for the 1969 un-propelled Goldschmied model ${ }^{(16)}$

\subsubsection{1982 Test Model}

The 1982 model was of the most interest because it contained the complicated internal flow, along with the boundary layer suction, the exit jet, and the interactions between all of the components. The structured grid, along with the fan on streamlines, can be seen in Figure 17 and Figure 18 below. One interesting note is that the internal geometry was not taken from Howe's test procedure (as can be seen in Figure 13) but from a smaller image reproduced in a paper by Goldschmied ${ }^{(15)}$. This shows the difficulty associated with trying to reproduce Goldschmied's data and the lack of information that was supplied from his testing. The aft section and slot geometry also seemed to differ slightly between sources. 


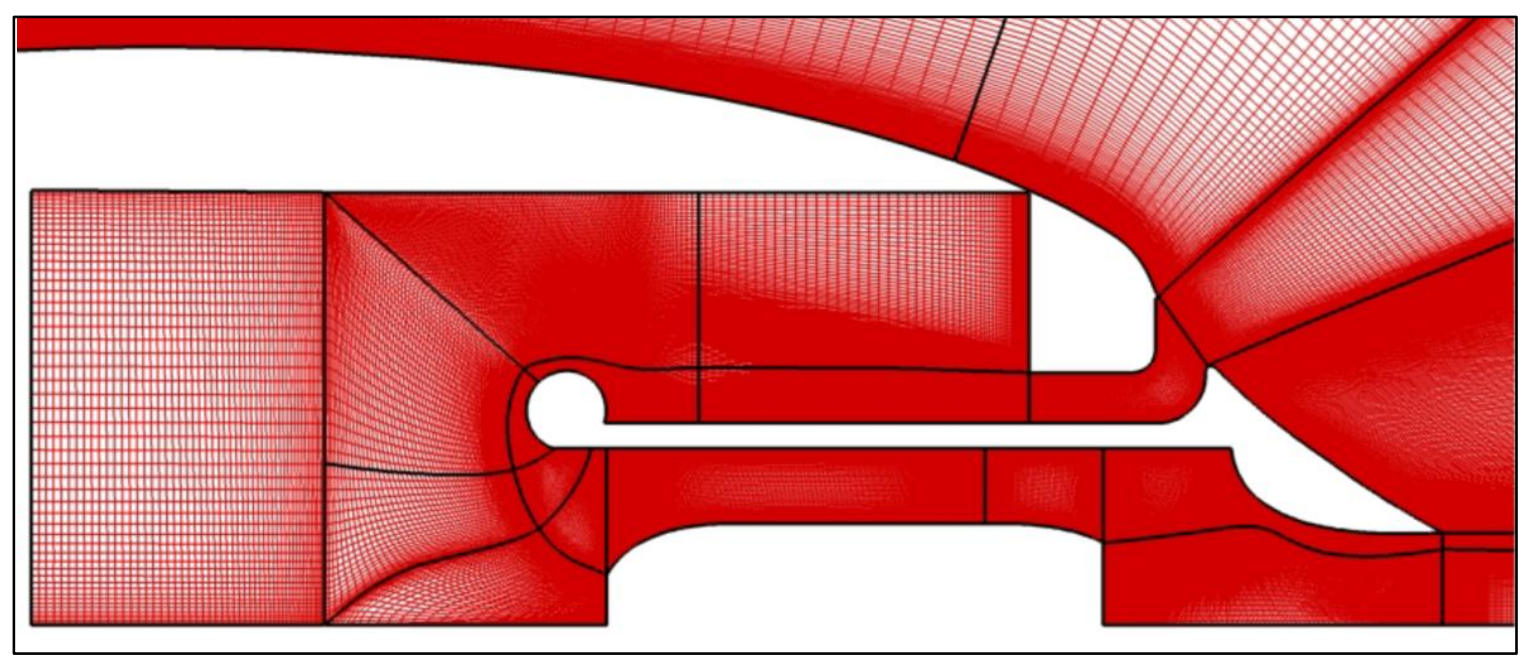

Figure 17: Medium structured mesh for the 1982 Goldschmied model $^{(16)}$

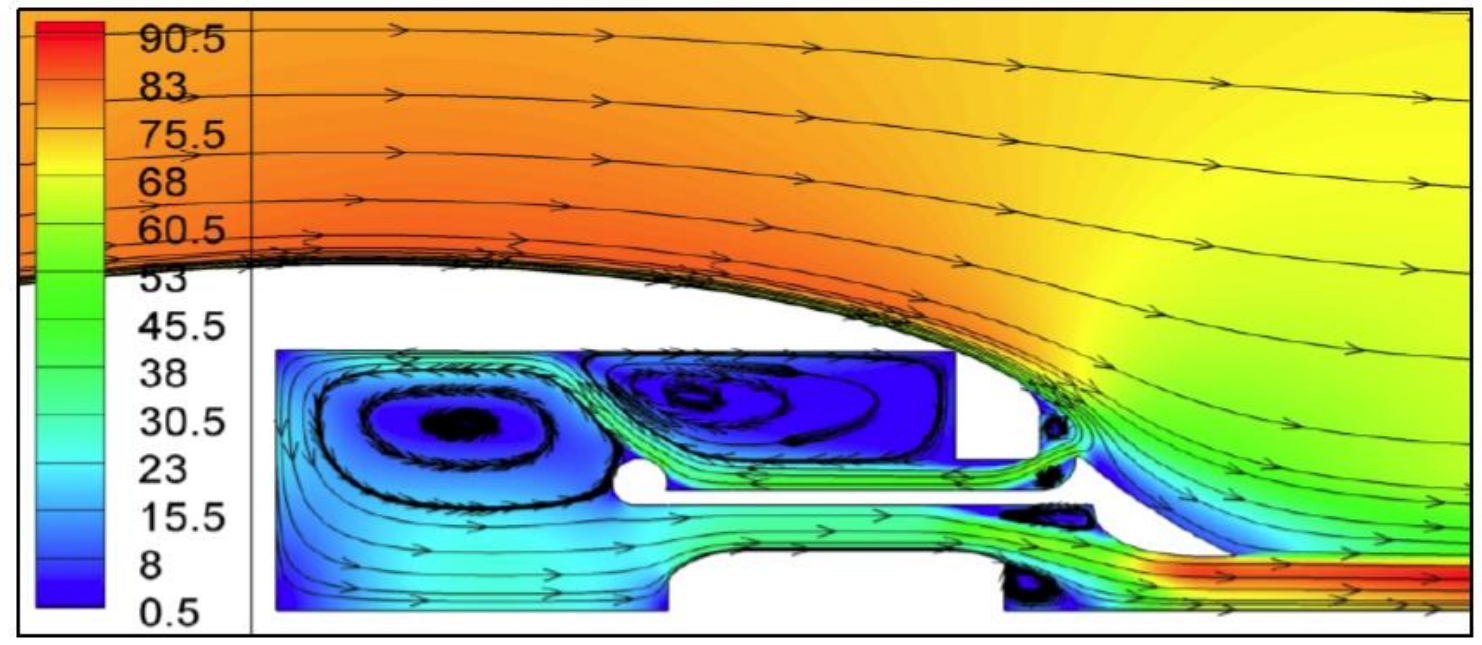

Figure 18: Velocity contour and streamlines for the propelled airship with attached flow ${ }^{(16)}$

For this model, three cases were run: a "fan off" and two fan on cases with varying suction rates. All three pressure distributions are shown in Figure 19 below and can be compared to Goldschmied's experimental results. 


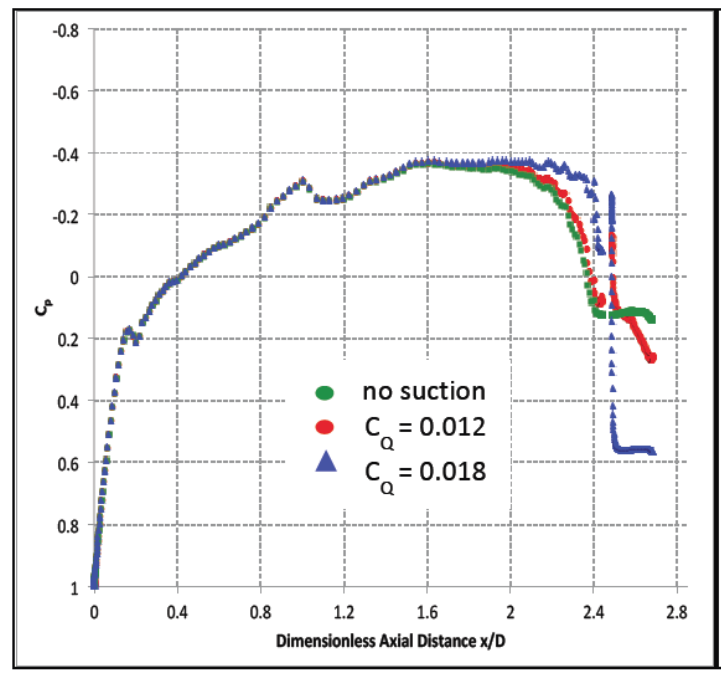

(a) $C F D$ results

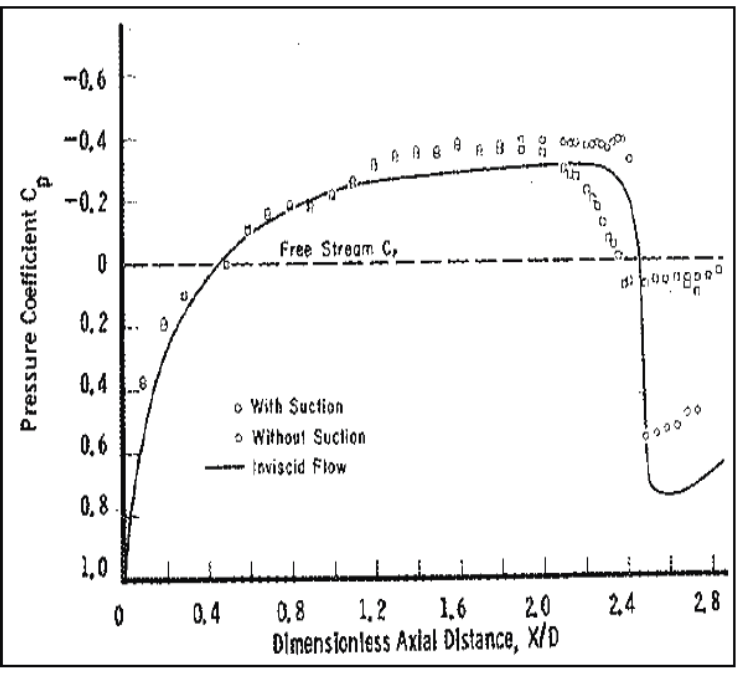

(b) Experimental results $\left(C_{Q}=0\right.$ and $\left.C_{Q}=0.012\right)$

Figure 19: Peraudo CFD (a) and experimental (b) Cp Distributions for the self-propelled BLC Airship with and without suction ${ }^{(16)}$

We can see that the $\mathrm{Cp}$ values for the fan off condition seemed to match up pretty well, decreasing to about $\mathrm{Cp}=-0.4$ at the maximum diameter and then increasing to about 0.1 for the separated region. The first fan on case with $C_{Q}$ of 0.012 was not enough to get the flow attached in the CFD like it showed in the experimental. The flow rate was increased until there was attachment on the aftbody. This wasn't reached until the flow rate was increased to $0.018,50 \%$ higher than the experimental value. This may have been due to the inaccuracies of modeling the slot and internal geometry or a poor flow rate measurement by Goldschmied. Regardless of the difference in flow rates, it seemed that in both the experimental and CFD cases, as the fan speed increased enough to attach the flow over the aft section, the overall drag decreased to zero. A summary of the results can be seen in Table 1. 
Table 1: Peraudo CFD results for the propelled BLC model

\begin{tabular}{|c|c|c|c|c|}
\hline & $\mathrm{C}_{\mathrm{Q} 5}$ & $\mathrm{U}_{5} / \mathrm{U}_{\infty}$ & $\mathrm{H}_{5} / \mathrm{H}_{\infty}$ & $\mathrm{C}_{D, \text { Wake }}$ \\
\hline Goldschmied & 0 & - & - & 0.034 \\
\cline { 2 - 5 } Experimental & 0.012 & 0.675 & 0.990 & 0.000 \\
\hline \multirow{2}{*}{ CFD } & 0 & - & - & 0.030 \\
\cline { 2 - 5 } Results & 0.012 & 0.712 & 0.993 & 0.023 \\
\cline { 2 - 5 } & 0.018 & 1.042 & 1.020 & 0.000 \\
\hline
\end{tabular}

Overall Peraudo et al. concluded that the embedded propulsor was not optimally designed and a good amount of improvements could be made by making that section of the model better. There were large uncertainties in the testing results that made it hard to compare to. Either better knowledge of the wind tunnel model and more accurate data collection was needed or an improved model needed for testing to get more accurate results. 


\section{Cal Poly's Goldschmied Model and Testing}

Over the past two years students Joshua Roepke and Nicole Thomason, from California Polytechnic State University, San Luis Obispo CA have constructed and tested a new Goldschmied propulsor in Cal Poly's 3x4ft low-speed indraft wind tunnel. The goal of the recent model and testing was to replicate Goldschmied's results and provide a transparent basis of knowledge for future study. The following sections summarize what Roepke and Thomason have done; for all details the reader is encouraged to read their theses, they can be found in references (17) and (18).

\subsection{Cal Poly's 3x4ft Wind Tunnel}

All testing for the New Goldschmied body was done in Cal Poly's 3x4ft indraft tunnel. The tunnel was constructed in 1974 and is mostly made from wood. It is powered by a $150 \mathrm{hp}, 440$ Volt three-phase motor that is connected to a nine-blade axial fan. A planform view of the tunnel can be seen in Figure 20 below.

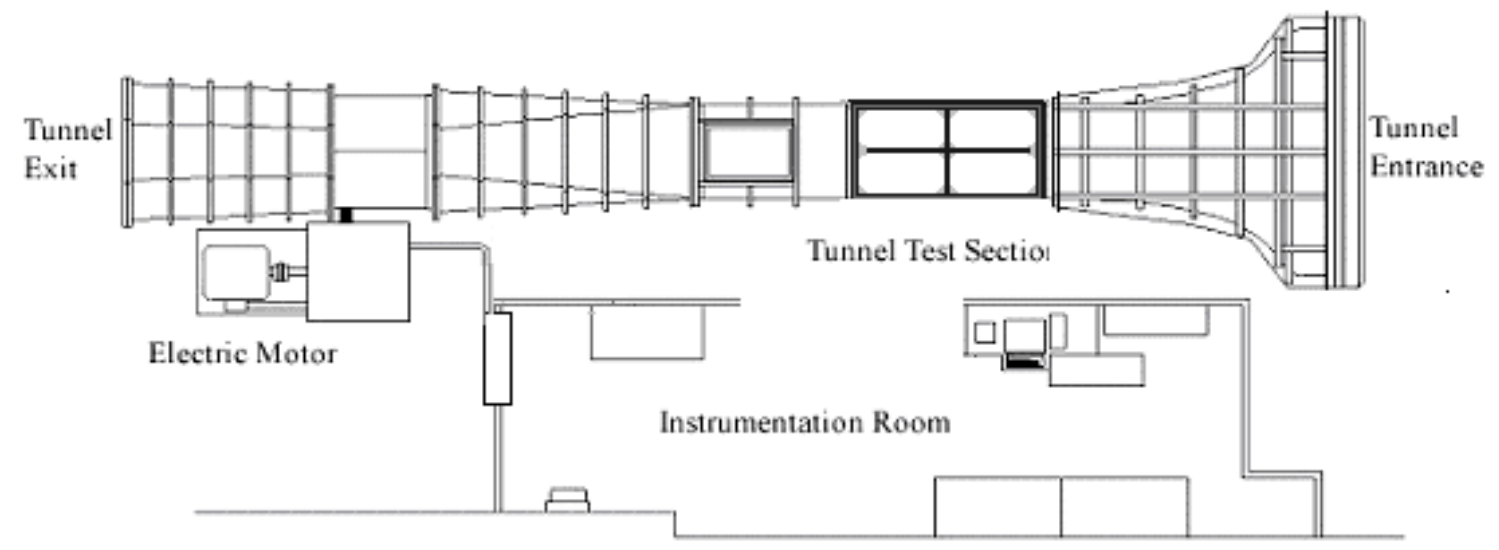

Figure 20: Top View of Cal Poly's 3x4ft Draw Down Wind Tunnel

An important note about the tunnel was that the center test sections were all of equal and constant area for the whole center section. There is no increase in area, as is sometimes done by reducing the chamfers in the corners. The constant area does not 
account for boundary layer growth that will effectively reduce the cross section of the tunnel. With this reduction the average velocity in the center of the tunnel will accelerate as it flows further downstream.

\subsubsection{Recent Tunnel Modifications}

Recently Cal Poly's wind tunnel went through an inlet screen replacement that greatly improved the flow in the test section. Greg Altmanns's wind tunnel test ${ }^{(19)}$, previous to any of the Goldschmied testing, found that they were getting results that were not what they expected. The problem was traced to a dirty and poorly sized inlet screen. This was easily seen when Altmann and Roepke conducted a flow visualization of the inlet. There were places where the inlet was nearly $100 \%$ blocked, leading to dramatic changes in flow direction, as seen in Figure 21.
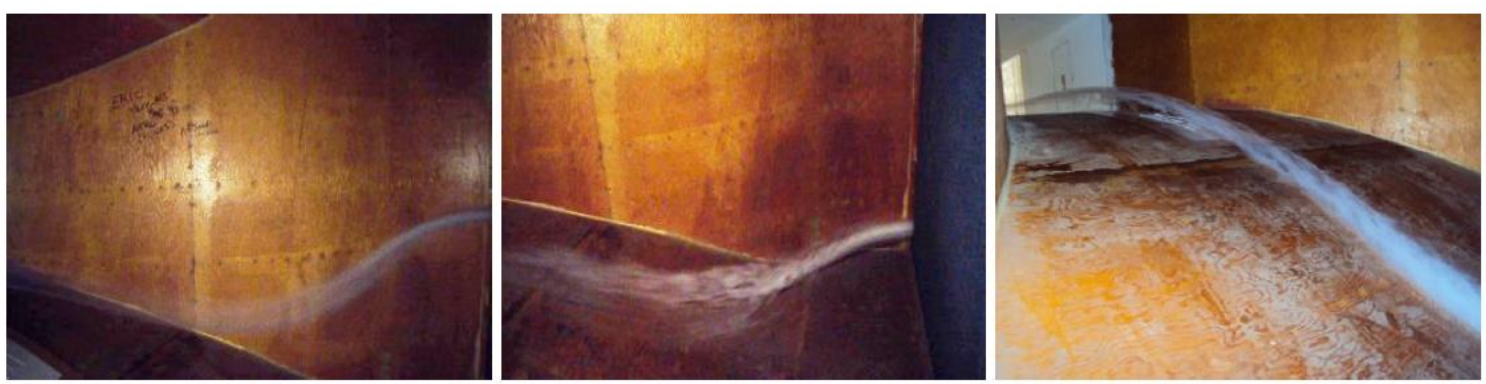

Figure 21: Wind tunnel inlet flow visualization before screen redesign ${ }^{(19)}$

This discovery led them to take apart the tunnel inlet, replace the poorly sized screens, cleaned the other screens and the rest of the inlet. The cleaning and redesign greatly improved the flow quality in of the inlet. The improved flow can be seen in Figure 22. 

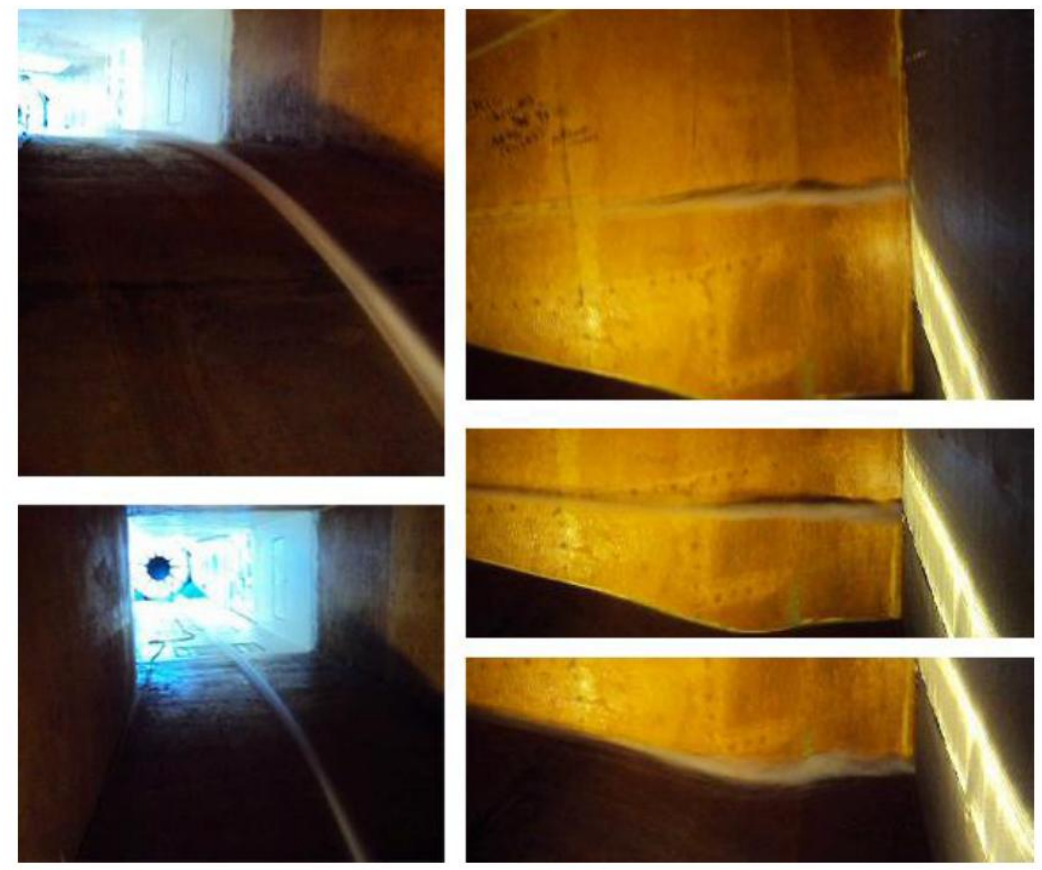

Figure 22: Wind tunnel inlet flow visualization after screen redesign ${ }^{(19)}$

After the new inlet was completed, a group of students completed a study of the flow quality ${ }^{(20)}$ at the center of the test section to find the velocity variation and turbulence intensity. They constructed a traverse that could travel in a vertical and horizontal direction to span the whole wind tunnel at a single station. From this they found that the total velocity variation was about $3 \%$ off the average velocity and a turbulence intensity of about $0.5 \%$ with a peak of about $2.7 \%$ at the top of the tunnel. The velocity and turbulence plots can be seen in Figure 23 and Figure 24. This discrepancy at the top seemed odd, but the data was reproduced weeks later when they re-evaluated the tunnel. The source of this anomaly is unknown, but since it is located near the top of the tunnel and not in the center of the test section we will assume that the overall average turbulence for our CFD calculations will be $0.5 \%$. 


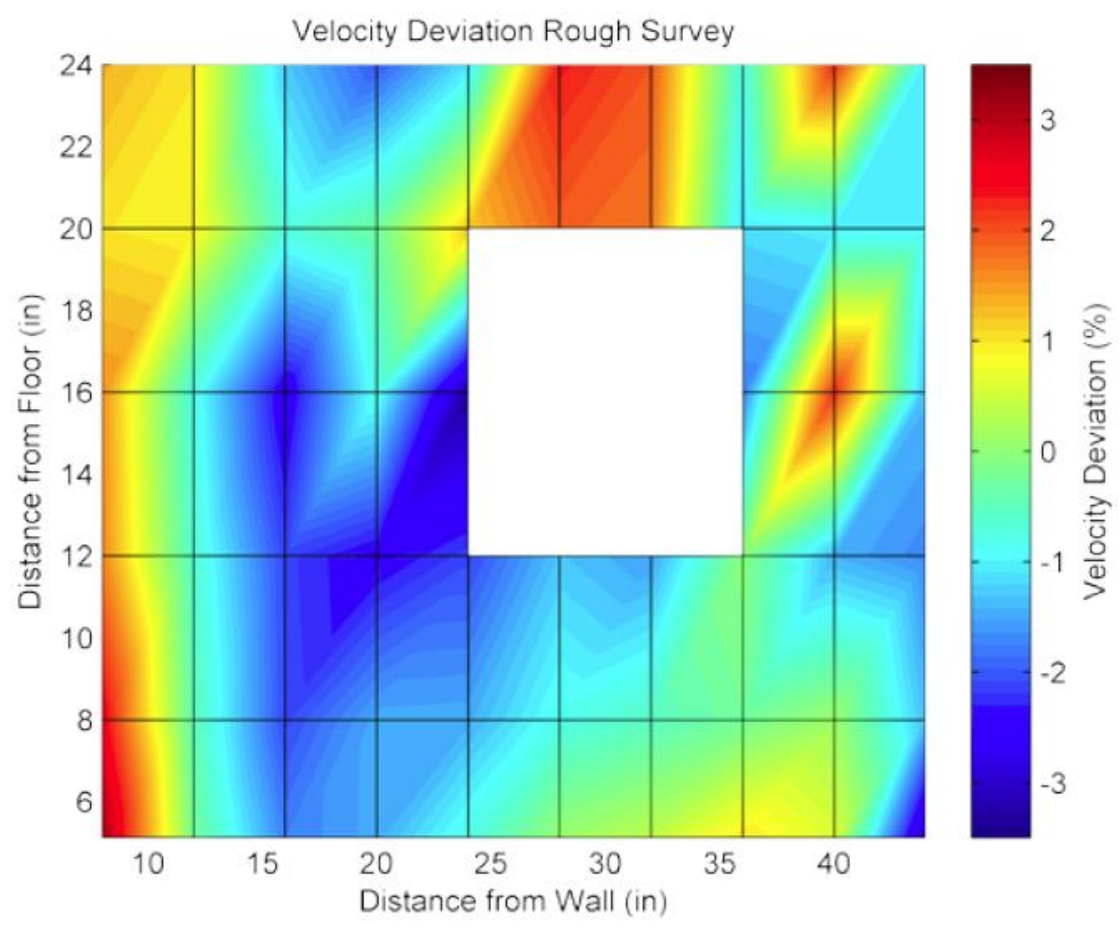

Figure 23: Velocity deviation in the improved Cal Poly wind tunnel course grid ${ }^{(20)}$

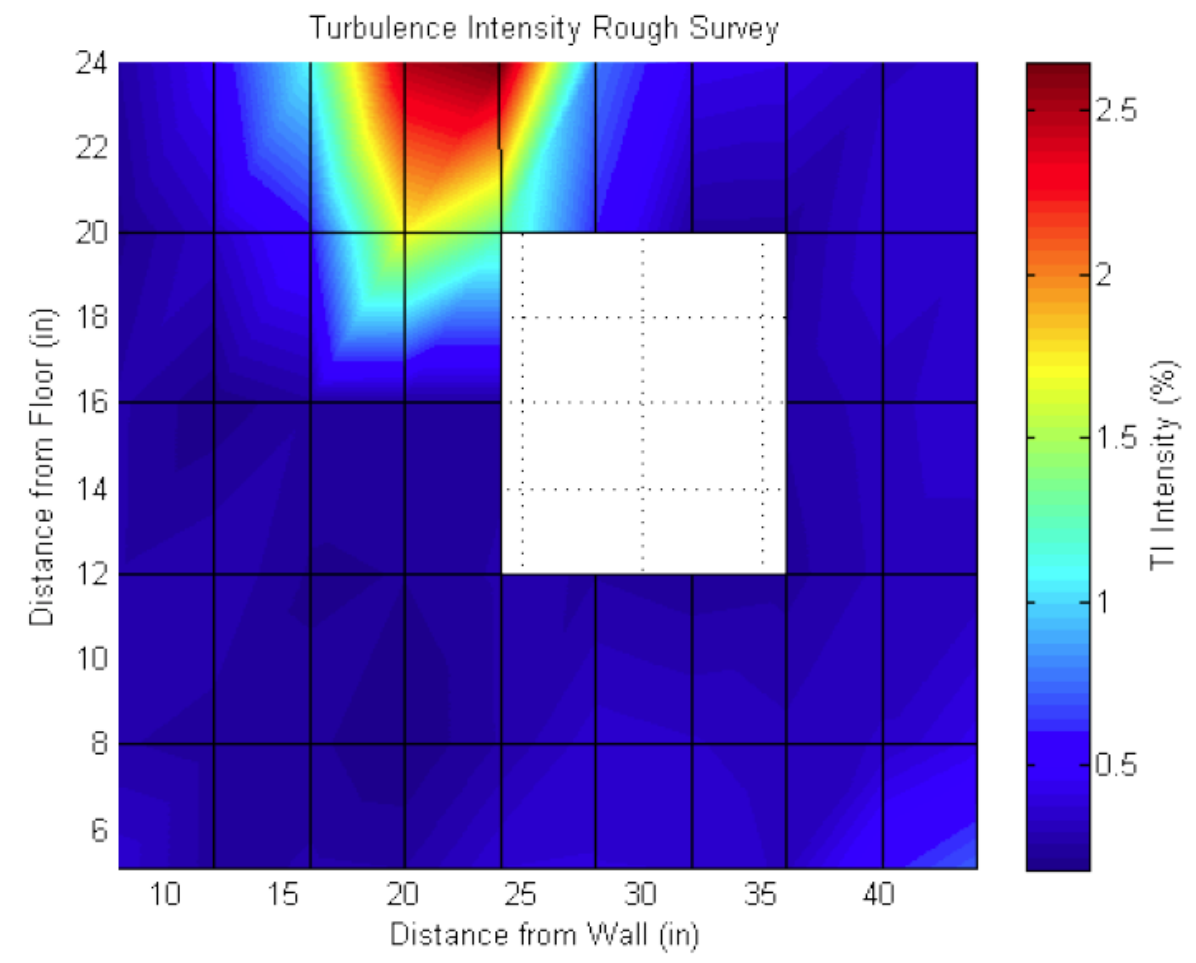

Figure 24: Cal Poly wind tunnel course grid turbulent intensity plot ${ }^{(20)}$ 
The blank spots in the figures above are sections that were not traversed in the course data collection stage; rather they were saved for the fine traverse. The fine traverses are not reproduced here, as there is no new information in them.

\subsection{Cal Poly Goldschmied Model}

Cal Poly's Goldschmied propulsor was designed to replicate Goldschmied's model with the least amount of changes to the outer body but to also improve the internal fan unit. The model had to be scaled down from the original 58"length and 20 " diameter to something smaller that would fit in Cal Poly's 3x4ft tunnel. Because of the smaller tunnel size the model was reduced so the maximum cross sectional area of the model was $9 \%$

of the tunnel cross sectional area. This was done to keep blockage effects down to a minimum, which was set at a maximum of a $5 \%$ increase in airspeed around the model. This sized the maximum diameter of the model to be 13.5 " and a length of 38.5 ". A comparison of this to previous models can be seen in Table 2 below. 
Table 2. Summary of the original Goldschmied models compared to Cal Poly's model ${ }^{(17)}$

\begin{tabular}{|c|c|c|c|}
\hline & 1956 Model & 1982 Model & Cal Poly's Model \\
\hline & & \begin{tabular}{l|l} 
short & Long
\end{tabular} & \\
\hline $\begin{array}{l}\text { Reynolds numbers (based on } \\
\text { length) }\end{array}$ & $4 \times 10^{6}-12 \times 10^{6}$ & $\begin{array}{c}3.2 \times 10^{6}-5.0 \\
\times 10^{6}\end{array}$ & $\begin{array}{l}1.34 \times 10^{6} \\
\text { (at } 20 \mathrm{~m} / \mathrm{s})\end{array}$ \\
\hline Tunnel Size & $7^{\prime} \times 10^{\prime}$ & $8^{\prime} \times 10^{\prime}$ & $3^{\prime} \times 4^{\prime}$ \\
\hline Length & $58.8^{\prime \prime}$ & $54.45 " / 57.17$ & $38.5 "$ \\
\hline Maximum Diameter & $20.0 "$ & $20.0 "$ & $13.5^{\prime \prime}$ \\
\hline $\begin{array}{l}\text { Location of Maximum } \\
\text { Diameter }\end{array}$ & $54.1 \%$ & $57.9 \% / 55.1 \%$ & $\begin{array}{c}55.1 \% \\
(21.23 " \text { from LE) }\end{array}$ \\
\hline Forbody Diameter at Slot & $11.5^{\prime \prime}$ & $11.5 "$ & 7.75 \\
\hline Aftbody Diameter at Slot & $9.62 "$ & $9.35 "$ & $7.00 "$ \\
\hline Exit Diameter & & $3.3^{\prime \prime}$ & $1.82 "$ \\
\hline Slot Location & $86 \%$ & $90 \% / 85.8 \%$ & $\begin{array}{c}86 \% \\
\text { (33.12" from LE) }\end{array}$ \\
\hline Body Volume & Not Available & $6.35 \mathrm{ft}^{3}$ & $1.89 \mathrm{ft}^{3}$ \\
\hline
\end{tabular}

The individual subsets of the model will be talked about in the following sections, but the final SolidWorks model can be seen in Figure 25. 


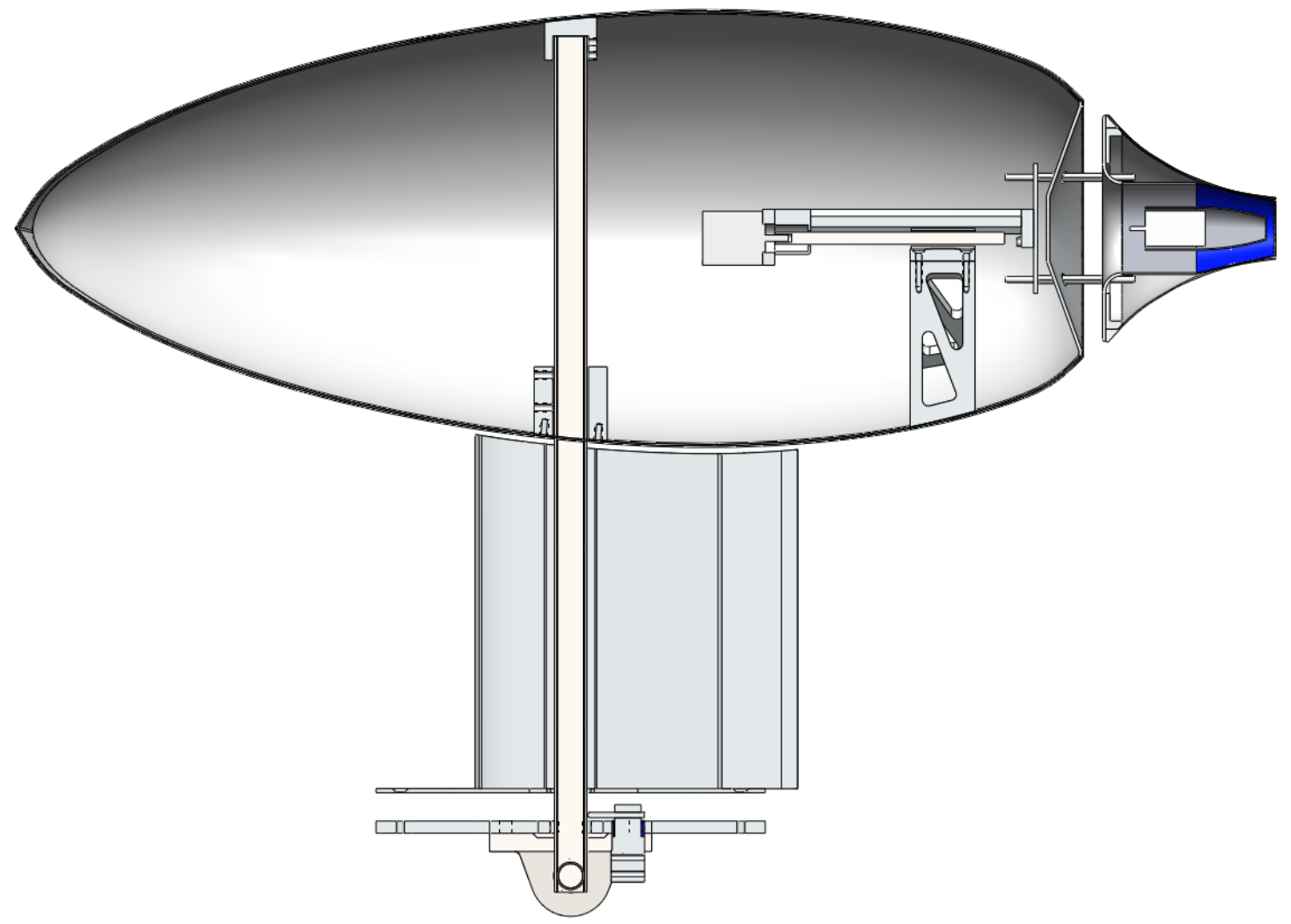

Figure 25: Final Goldschmied SolidWorks Model

\subsubsection{Outer Geometry}

Cal Poly's model was based on Goldschmied's data. The main problem with this approach was the lack of an exact set of coordinates. The geometry is based on the locations of the pressure ports listed in each of the test papers. These values were not exactly the same and the aft section varied between tests. The data points can be seen below in Figure 26.

For the forebody, it was observed that its geometry is reported as nearly the same for all tests, as the same physical model was used for all tests. These values were averaged to find the current fore body. For the aft body, the longer of the open aft bodies 
was chosen. This was chosen because it was both easier for Goldschmied to get aft body attachment and seemed easier to mount the ducted fan and motor internally.

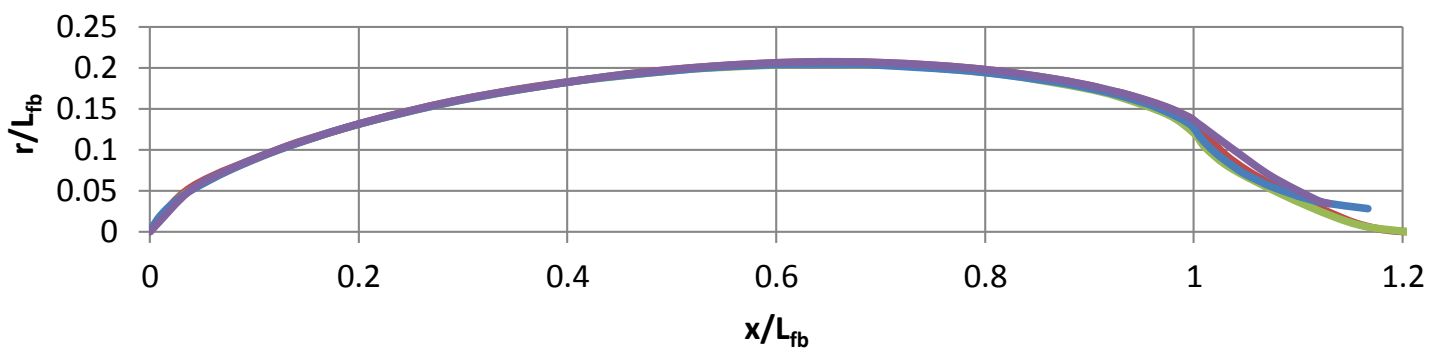

Figure 26: Comparison of body shapes used in Goldschmied's testing non-dimensionalized by forebody length ${ }^{(7)(9)(10)(21)}$

These points were then reduced and smoothed out to get a shape that was both representative of Goldschmied's tests and would eliminate any discontinuities or bumps. Roepke and Thomason's theses contain more detail on their geometry selection and refinement.

\subsubsection{Slot Design and Construction}

The slot is the most important feature, yet it seemed to be the least documented geometry in the whole model. There were no values for the slot besides the outer opening, and even that was based on simple measurements, mainly just the slot width and inlet diameter with nothing documented about the exact routing. This made reproducing the inlet and exit somewhat of an unknown, and most of the information was found in drawings from the original models, as can be seen in figures on pages 11,12 , 14,18 , and 19 of this report.

With this lack of knowledge and with suggestions that the original slot was less than ideal, the slot for the new model underwent a total redesign. The aft section was mounted on a horizontal traverse that allowed the slot gap to vary in width. There was a variable cusp design that allowed three different geometries to be tested. Inside the slot, 
the front face was concave in to allow a constant radial cross sectional area as the flow moved in. The motor was mounted internally to the aft section and the torque and force were measured through a set of sensors.

\subsubsection{Cusp Design}

At the end of both the 1969 and 1982 tests, Goldschmied claimed that an added 'Ringleb' cusp added a huge increase in performance and helped to stabilize the flow. Because the initial ring was formed by molding clay around the suction slot inlet by hand, the exact geometry is not known. Understanding this, the closest reproduction of a cusp drawing is seen in Figure 9 on page 14 above. From Thomason's thesis, we see that there were three main cusp geometries tested on the Cal Poly model. Cusp B, the center of in Figure 27 below, was taken directly from Goldschmied's original sketch.
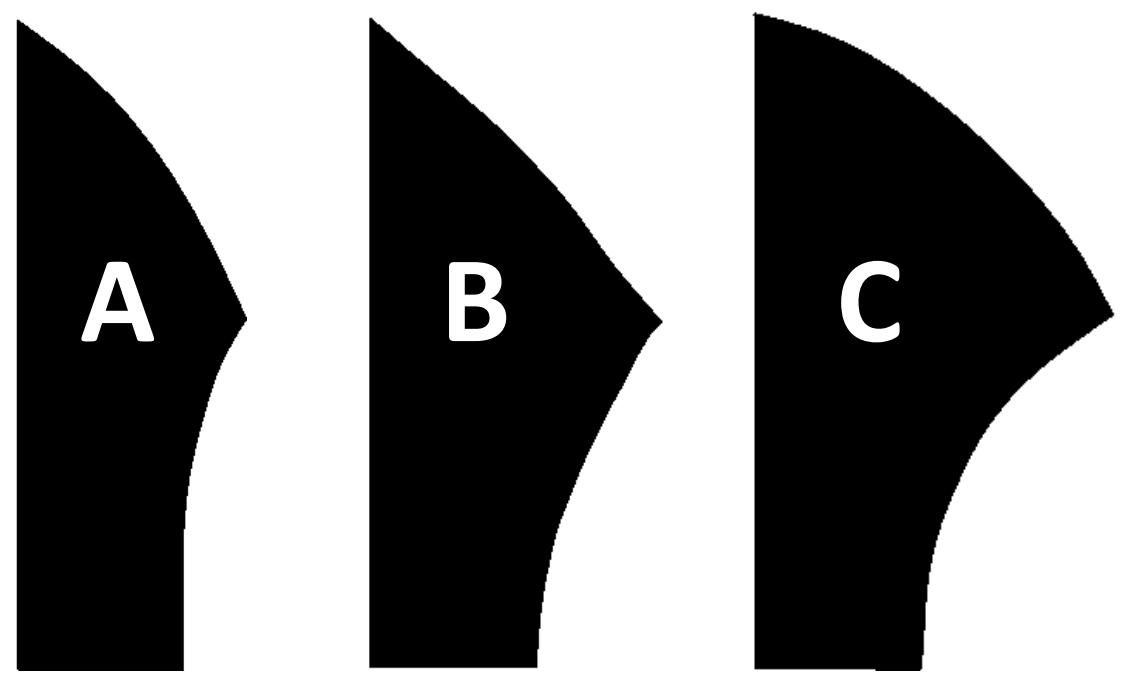

Figure 27: Three cusp geometries that were constructed for the Goldschmied propulsor ${ }^{(18)}$

The other two cusps were variations of the first one, with Cusp A protruding less into the flow and Cusp C protruding a lot further and turning a lot more. The goal with these cusps was to get a basis of what worked and what didn't and hopefully give an idea on how to move forward in the future. 


\subsubsection{Fan Unit}

The goal of the fan unit for the new model was to find something that was small enough to fit into the aft section of the model and still be able to deliver enough suction and flow rate to ingest enough of the boundary layer to re-attach the flow on the aft section of the body. Having the fan mounted in the aft section got around the big losses in Goldschmied's original testing with his $180^{\circ}$ turns. Also the motor was highly monitored, to determine how much power was really needed to attach the flow. The power supply was able to output the voltage and current that it was supplying. In addition, the model was fitted with load cells on the aft to measure force and torque. These measurements allowed one to measure the power delivered to the air, the power delivered to the motor and speed controller, and thus the efficiency of the whole system. The fan module can be seen below in Figure 28. The fan unit was capable of delivering around 500 watts at its maximum setting.

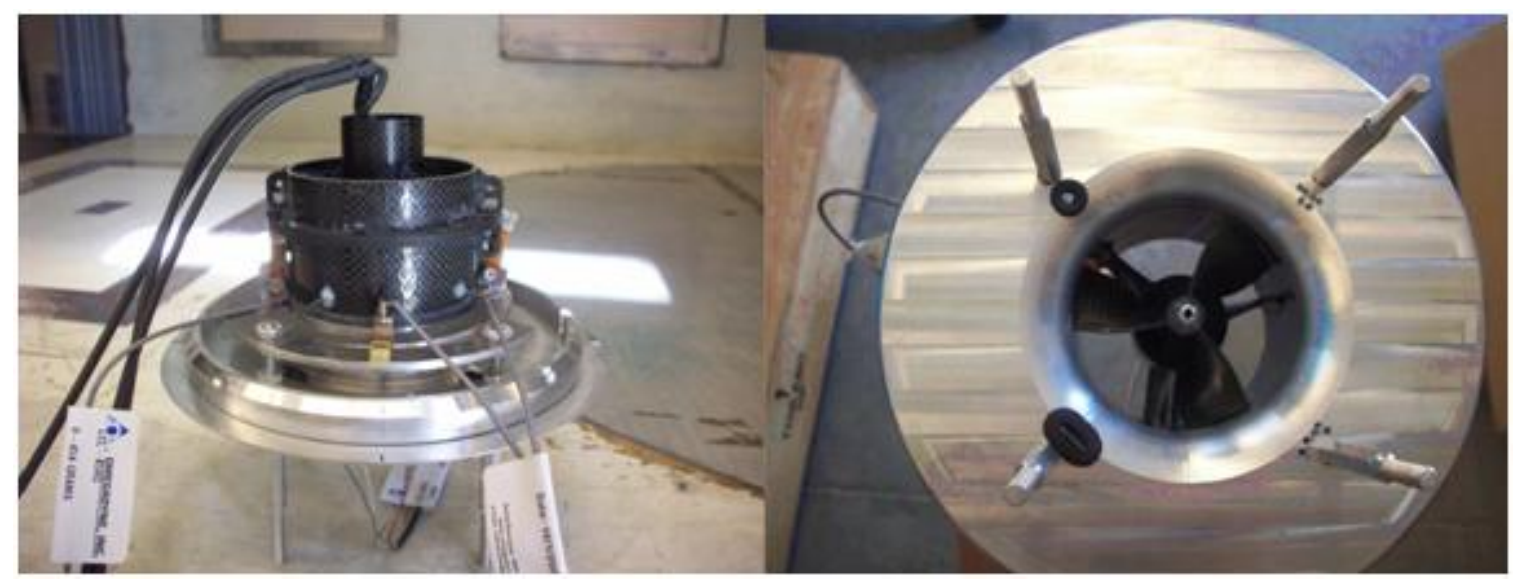

Figure 28: Ducted fan propulsion unit and inlet rear face mounting

\subsubsection{Tunnel Mounting Method}

The mounting for the Cal Poly Goldschmied body did not use the standard 3x4 $\mathrm{ft}$ tunnel sting, this was excluded because the sting was located at the rear of the tunnel and for testing Roepke and Thomason wanted the cleanest air possible, which was soon 
after the contraction. Also the sting was not strong enough to support the full weight of the 38 " model. With these considerations, it was decided to mount the model at the front of the tunnel on a vertical steel bar that was allowed to rotate at the base through the means of two flexure joints. The flexure joints allows for a friction free way to rotate, which, when coupled with a load cell at the base, allowed one to measure the axial force on the model.

\subsubsection{Propulsor Construction}

The main body of Cal Poly's Goldschmied propulsor was made out of carbon fiber, which was made from laying-up in a female mold. This process allowed a relatively complex shape that would be light, stiff, and had a lot of internal space to allow for mounting of components. A similar process was used to mold the aft body. A majority of the rest of the components were machined out of an aluminum billet. This was because it was light, easy to machine, and allowed for easy integration into the vehicle. An example of this was with the cusps: they were machined out of a single piece that allowed the cusps to be mounted to the back section of the fore body by means of a slot and tabs that can be inserted and then rotated to secure it.

\subsection{Testing Results}

The testing from Roepke and Thomason's research was run in multiple stints over the 2011-2012 school year. The model was brought in and out of the tunnel a few times so the wind tunnel could be used by classes during the year. The initial testing was used to set up and run the model. Throughout the testing there were many changes to improve the data quality and we are mainly concerned with the latest data set collected in March. This is the data that will be directly compared to the CFD calculations. 


\subsubsection{Operating Conditions}

Cal Poly is located in San Luis Obispo, CA, at an altitude of approximately $300 \mathrm{ft}$ MSL and about 12 miles from the coast. Because of this, the wind tunnel sees very temperate and consistent atmospheric values. The actual test values were very close to standard sea level (SSL) operating conditions so SSL conditions are assumed. The SSL values can be seen in Table 3. Actual test temperatures fluctuated between 56 to $61^{\circ} \mathrm{F}$, a density around 0.00235 slugs $/ \mathrm{ft}^{3}$, and a pressure around $14.72 \mathrm{psi}$.

Table 3: Standard Sea Level operating conditions ${ }^{(22)}$

\begin{tabular}{|l|l|l|}
\hline Pressure & $101.3 \mathrm{kPa}$ & $14.7 \mathrm{psi}$ \\
\hline Density & $1.22 \mathrm{~kg} / \mathrm{m}^{3}$ & $0.00237 \mathrm{slugs} / \mathrm{ft}^{3}$ \\
\hline Temperature & $15^{\circ} \mathrm{C}$ & $59^{\circ} \mathrm{F}$ \\
\hline Gas Constant & $287 \mathrm{~J} / \mathrm{kg}-\mathrm{K}$ & $1716 \mathrm{ft}-\mathrm{lb}_{\mathrm{f}} / \mathrm{slug}^{\circ}{ }^{\circ} \mathrm{R}$ \\
\hline Kinematic Viscosity & $1.46 \mathrm{e}-5 \mathrm{~m}^{2} / \mathrm{s}$ & $1.58 \mathrm{e}-4 \mathrm{ft}^{2} / \mathrm{s}$ \\
\hline
\end{tabular}

\subsubsection{Thomason's Results for $30 \mathrm{~m} / \mathrm{s}$ Testing}

The data presented in Thomason's thesis "Experimental Investigation of Suction Slot Geometry on a Goldschmied Propulsor" was all run at $30 \mathrm{~m} / \mathrm{s}$. It was found out later, and described in the next section, that at this tunnel speed the propulsion unit was not able to inhale enough of the boundary layer to fully reattach the aft section. This can be seen in Figure 29 where there is partial attachment, but the flow is still chaotic and there are gaps in the plow, indicating recirculation pockets. 


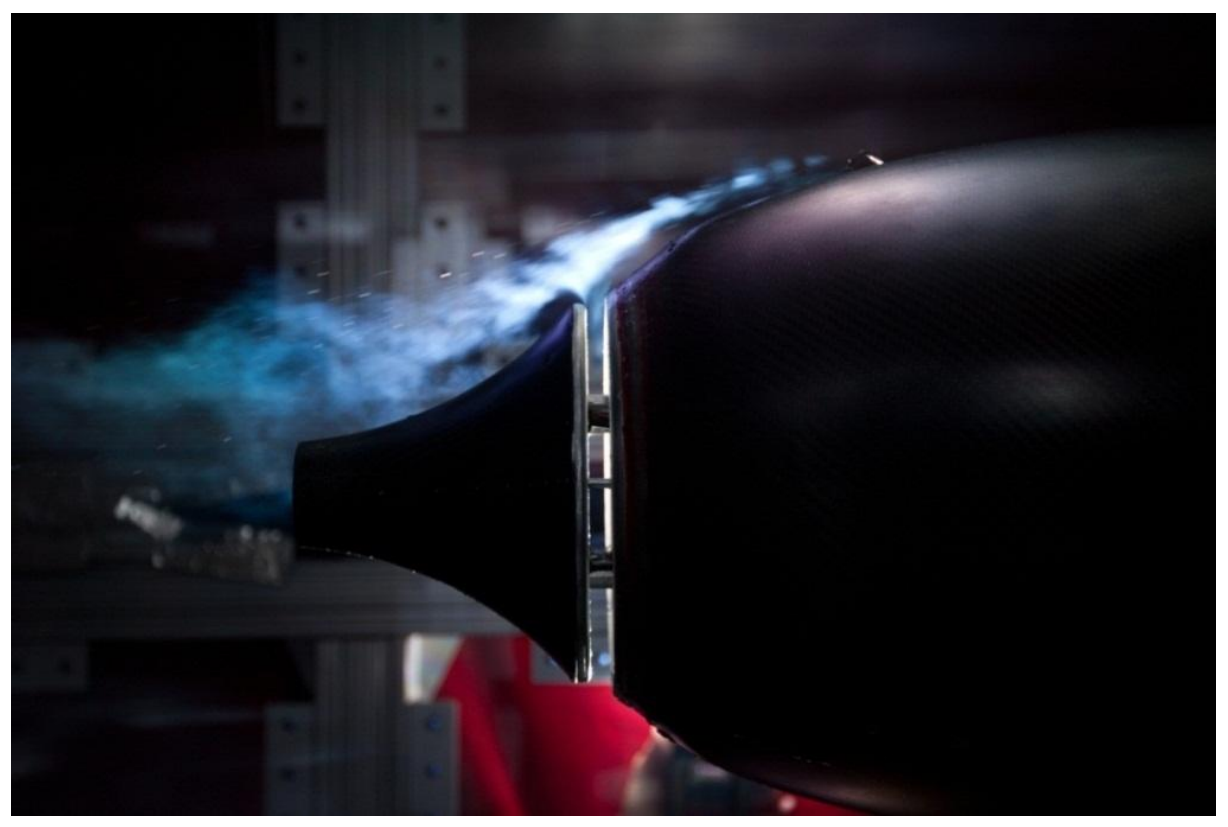

Figure 29: Flow visualization with Cusp $C$ and fan at full power showing separation

Thomason also concluded at the end of her thesis, "The errors [bars] make it clear that between the different cusps and different fan speeds, there is no meaningful difference in the pressure drag. Figure 68 [in her report] suggests that adding a cusp to the suction slot creates no meaningful reduction in drag." From these results, we are not going to be comparing CFD to the $30 \mathrm{~m} / \mathrm{s}$ results, as they all seemed to be separated and showing very little changes between settings.

\subsubsection{March Re-Testing at $20 \mathrm{~m} / \mathrm{s}$}

In March 2012, the new model was set up again, and this time tufts were added to the model. The tufts allowed us to see at what fan setting allowed for reattachment of the aft section. From this testing it was found that the tunnel speed had to be slowed to $20 \mathrm{~m} / \mathrm{s}$ to guarantee full attachment for the fastest fan speeds. This was reassuring knowing that the data being collected was capturing the re-attachment of the flow over the aft section, as intended. Because of this reattachment, the pressure recovery on the aft section had greatly improved, which can be seen in Figure 30 below. This data set 
captures the fan ranges that contain the flow characteristics that we are looking for. This will be the data with which the CFD modeling will be compared. It should also be noted that this paper is not going to reproduce all of Roepke's results, as those can be found in his final thesis.

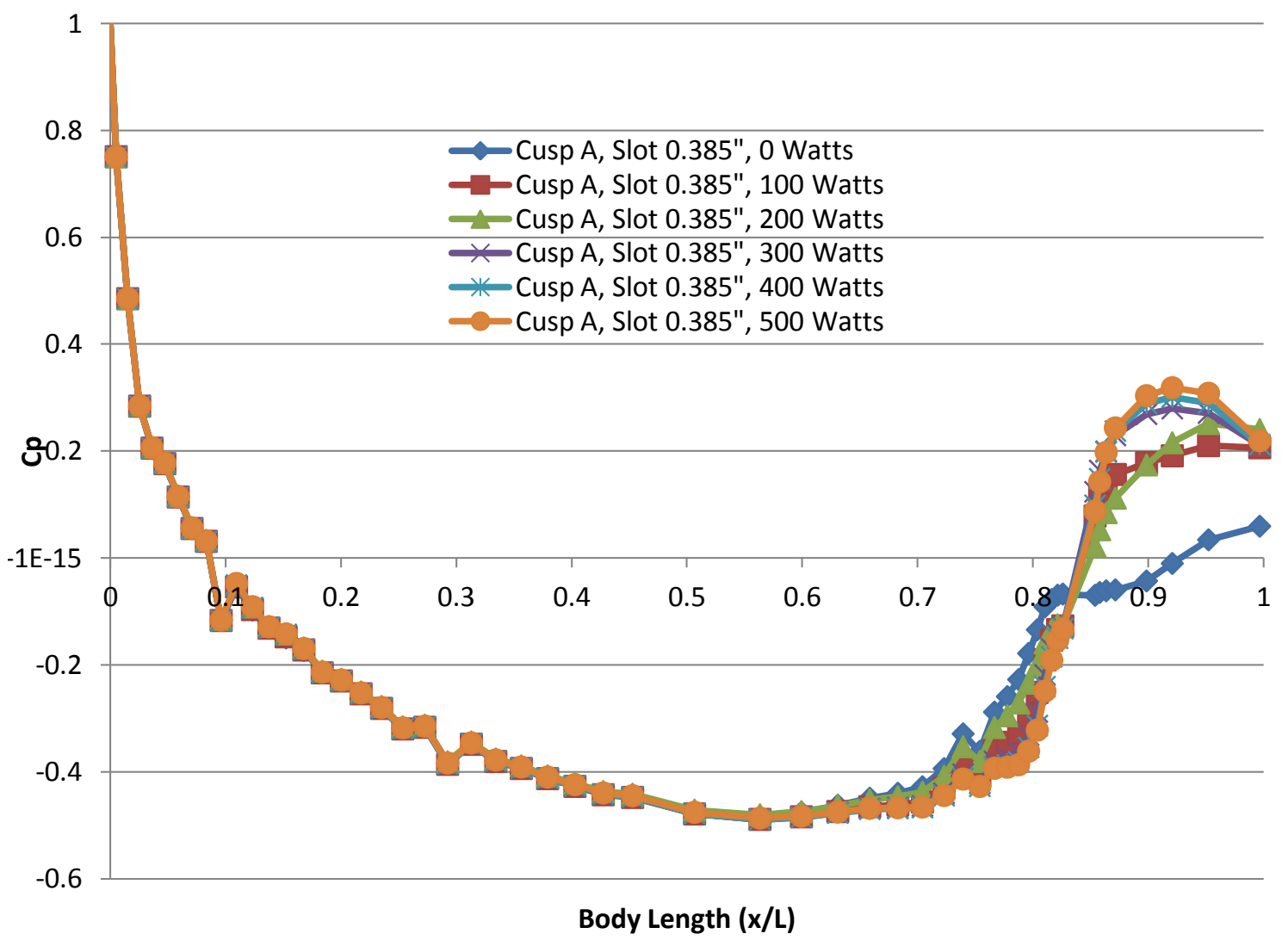

Figure 30: Experimental results from the March 20m/s re-testing 


\section{Computational Fluid Dynamics Approach}

The computational fluid dynamics goal was to see if/how well FLUENT, a commercial CFD software application, is able to capture the flow around a Goldschmied body. Because of this learning process, it took a lot of iterations to come to a finalized model. It entailed many loops of setting up the geometry, creating a mesh, and then solving to see what worked and what didn't. Because of this it was a highly iterative process, starting at a really simple model that used many default settings that eventually evolved into a more sophisticated model and with modified settings. All of this was done with the goal of trying to get the most accurate solution. The final CFD model was a simplified axisymmetric model that included important features from the real model while excluding smaller features.

\subsection{Model Component Simplifications}

Initial geometry models were very simple, starting with the outer mold line and a small inlet and outlet for the fan. The first models did not model the flow as it traversed the internal section. As the model iterated, components were added as their importance became apparent. At the end of the process, the axisymmetric model included the outer mold line, an accurate slot geometry including the intricate cusp shape and back wall, fan body, motor, and exit geometry. The main components that were not modeled were geometries that could not be modeled in a "2-D" axisymmetric case. These geometries are described in more detail in the following section.

\subsection{Axisymmetric Model}

A 3-D model was considered, as it would allow for greater detail that an axisymmetric model could not capture. These details include modeling strut interaction, aft body mounting bolts, fan mounts, rear pressure tubing, temperature gauges in the 
flow, wires, and other small items that were in the real model that could not be modeled in an axisymmetric case. These details seemed small, as they are mostly all internal, compared to the larger effects to be found and would not be worth the extra time need to grid and solve a fill 3-D solution. So an axisymmetric model was used. It was hoped that this would produce results comparable to the experiment and if it was found out after initial test cases that this could not adequately model the flow, then a 3-D model would be considered.

\subsubsection{Axisymmetric Tunnel Simulation}

Initial CFD runs showed that using an axisymmetric freestream model did not match up to experimental data as well as hoped. The first thought was that wind tunnel walls and set-up was the main reason for this difference. To understand the difference better, a simple axisymmetric wind tunnel was constructed around the CFD model. This was done because it was relatively simple to modify the grid and outer boundary conditions to simulate wind tunnel walls. The initial wind tunnel CFD model was modeled as a straight tunnel. The length of the section in front of the model was determined to have the same boundary layer thickness as to what is in the wind tunnel.

When looking at the mounting of the physical model in the $3 \times 4 \mathrm{ft}$ wind tunnel it was noted that the nose of the model was nearly at the exit of the contraction. Observing this, it was questioned what effect the model being that close to the contraction was. Because of this it was decided that a preliminary model of the wind tunnel would be constructed and ran. It was then compared to the straight tunnel to see how the contraction changed the flow. More details of this can be seen in section 8.3.2, Tunnel Effects. 


\section{Computational Model Geometry Generation}

The computational geometry used for solving needed to be as accurate as possible to the real model if it were to replicate the results. Significant effort was put forth to find the best and most accurate geometry. The following sections describe this effort.

\subsection{Geometry Sources}

The final geometry came from a few different sources: point files, SolidWorks solid model, and the actual model. The next sections categorize what components came from what source and how they were integrated together.

\subsubsection{Point Files}

The outer geometry was created from a combination of sources, as seen in section 3.2.1. The final smooth geometry came out to a set of about 30 data points, these data points were then used to create the solid model which led to the real model. Since there was no good way to measure the exact geometry of the real model, it was decided to use the set of 30 data points and create the CFD geometry around this.

\subsubsection{Solid Model}

The SolidWorks solid model was used for a majority of the internal slot geometry modeling. This was because it was easier than measuring the real model and since a majority of the components where machined from the solid model, the differences were expected to be small. Figure 31 shows the solid model that was used, the connection ring and cusp are hidden for simplicity. 


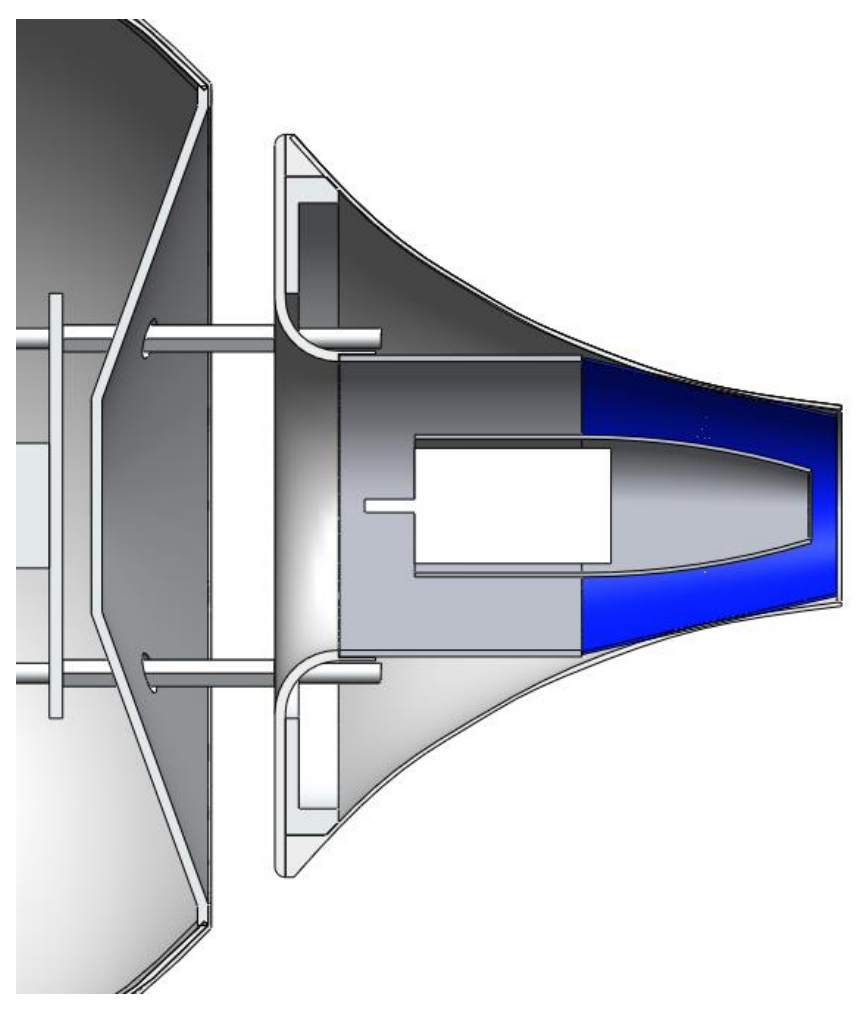

Figure 31: SolidWorks model used to model the internal slot geometry

Using the solid model did not come without issue, as the solid model was not exactly reproduced in the actual wind tunnel model. This can be seen in Figure 32, where the connection ring in the solid model is not precisely tangent to the fore body and is also not exactly aligned with the cusp. This was not the case in the real model, as the cusp and connection ring were closer to the same size and wax was used to fill in any non-tangent section. The solution to this was to not follow the exact SolidWorks geometry, but to create smoother geometry that more accurately represented the smoothed out section on the real model. 

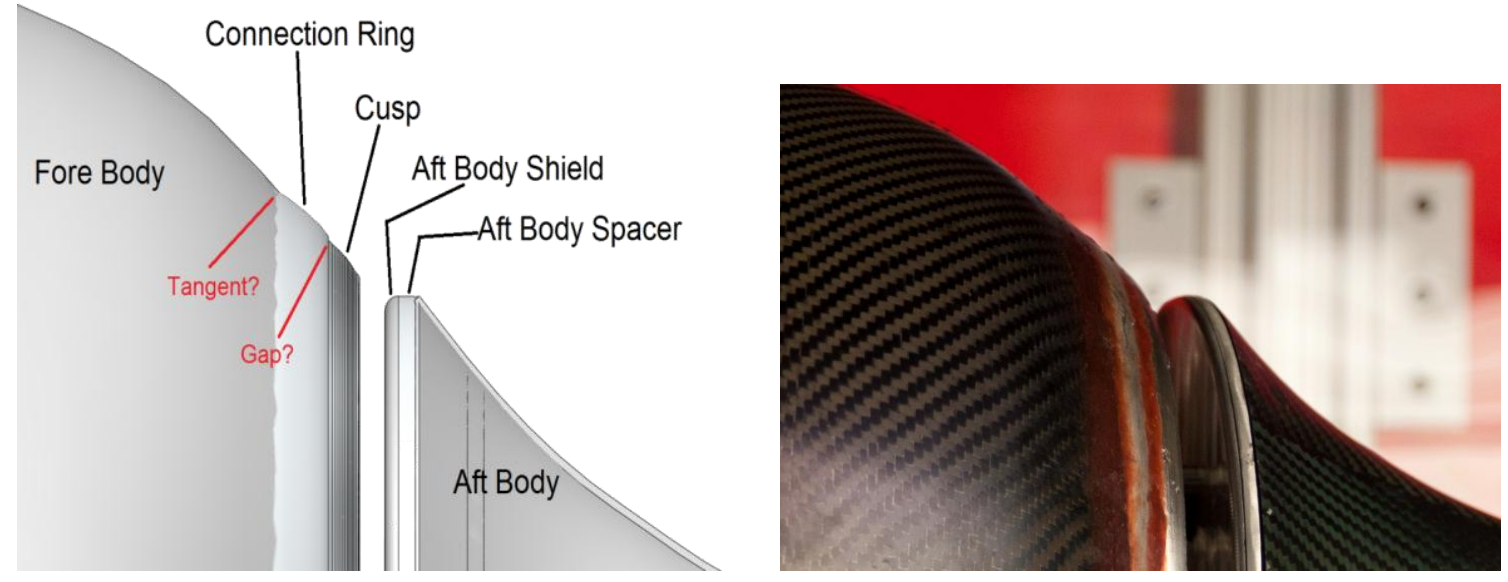

Figure 32: Discrepancies between the solid model connection ring and cusp and the final wind tunnel model

SolidWorks was also used to find the pressure port locations on the fore body. This was the most accurate method besides actually measuring them. Comparing the SolidWorks model to measurements of the actual model, the locations of the fore pressure ports match up well and are in agreement.

\subsubsection{Physical Cal Poly Model}

Finally the physical model was consulted to find any discrepancies between the previous methods described and the actual model that was being run in the tunnel.

A similar method in SolidWorks was used to find the fore body pressure ports, as described above, was used to locate the pressure ports on the aft body. This gave locations that did not seem correct. To fix this, a thin piece of foam was cut to fit the aft body geometry and then the pressure ports were located. The fitted piece can be seen in Figure 33 below. This was then brought onto the computer and a plot digitizer was used to find the exact location of each port. 


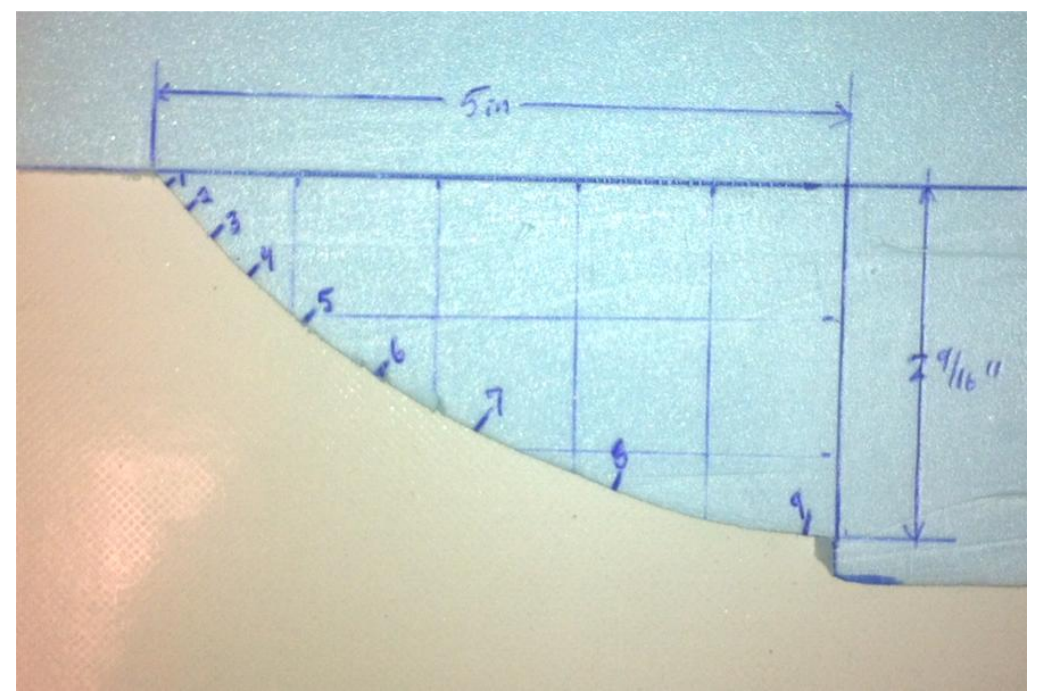

Figure 33: Pressure port locations on the aft body

After further inspection of the physical model it was found that there was a small 0.035 " lip at the start of the aft body immediately after the slot. This discovery can be seen below in Figure 34. This was a result of the carbon fiber aft body being slightly larger than the aluminum ring that it mounted to. The lip was not present the whole circumference, but it protruded for a large section that was centered around the pressure ports. This was a very important part of the model and any small change had a large effect on the flow on the aft body, so it was decided to add this small protrusion to the CFD model. 


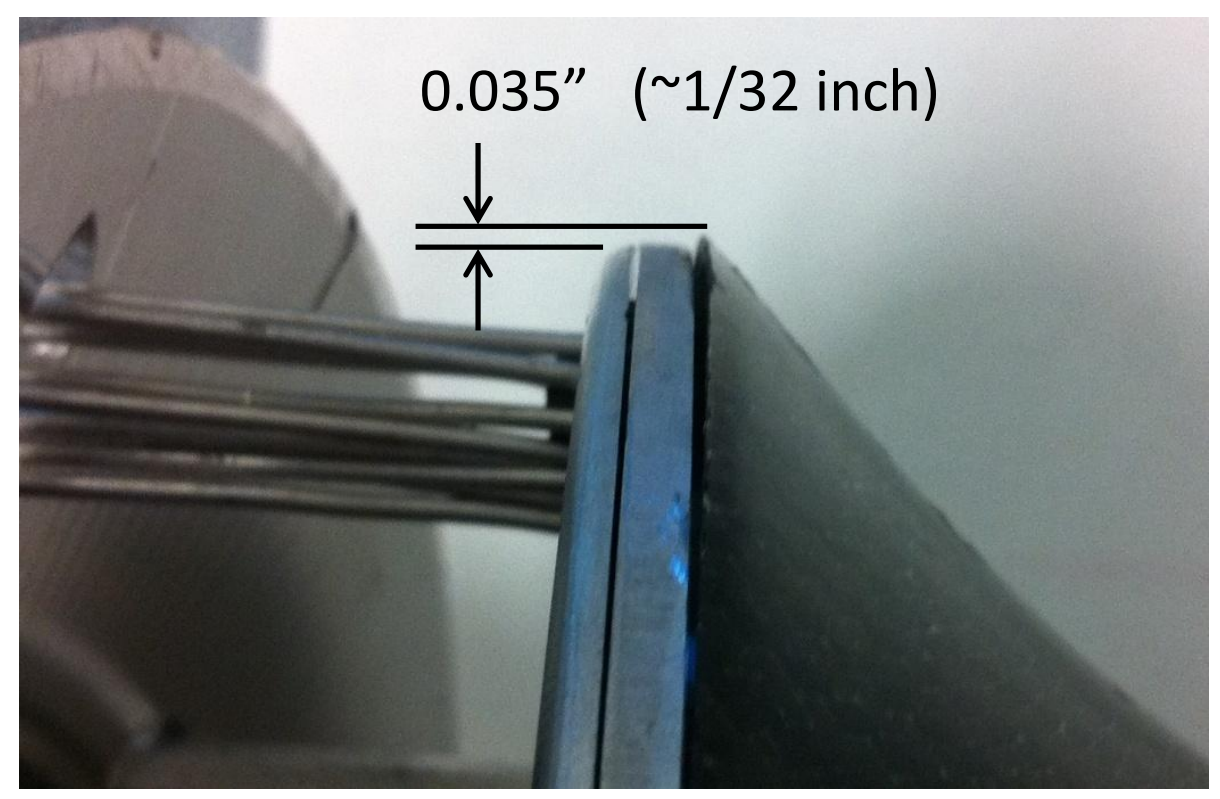

Figure 34: Lip on the aft section of the wind tunnel model

The final close-up of the CFD slot entrance, with cusp A and the lip modeled can be seen in Figure 35 below.

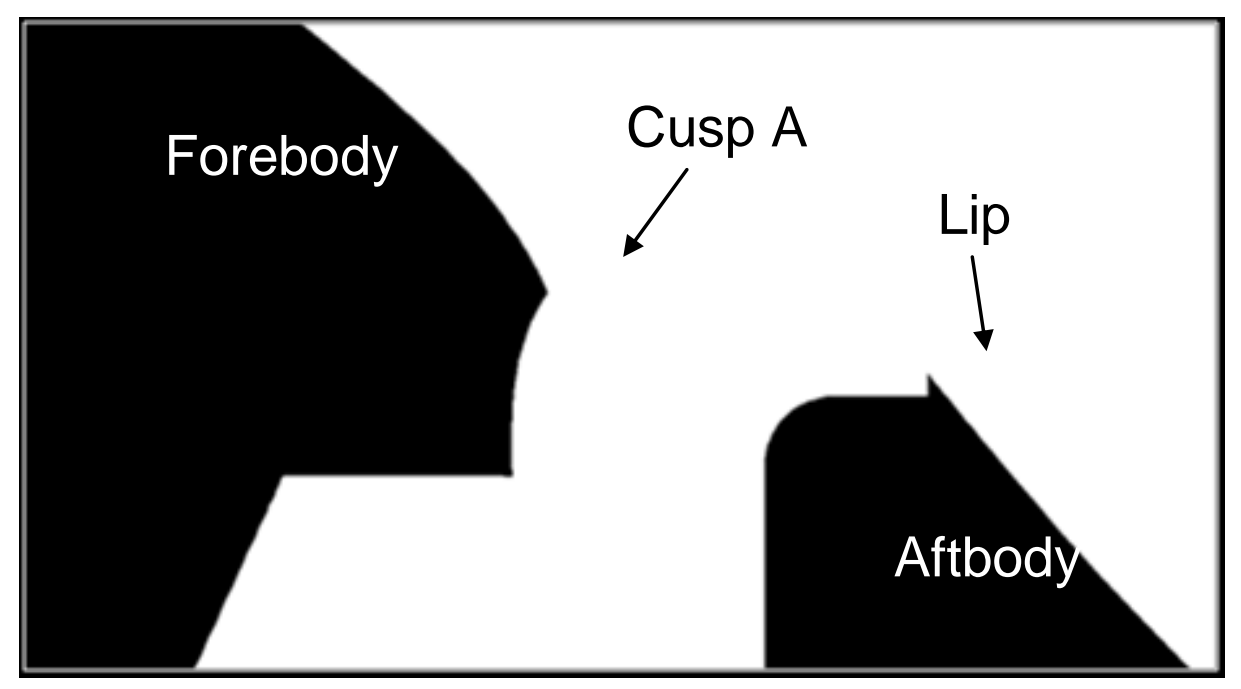

Figure 35: Final slot geometry with cusp A and aft lip modeled

\subsection{Final Geometry}

Putting all these three sources together, we get the final model geometry, as seen below in Figure 36. The final coordinate points can be found Appendix A 


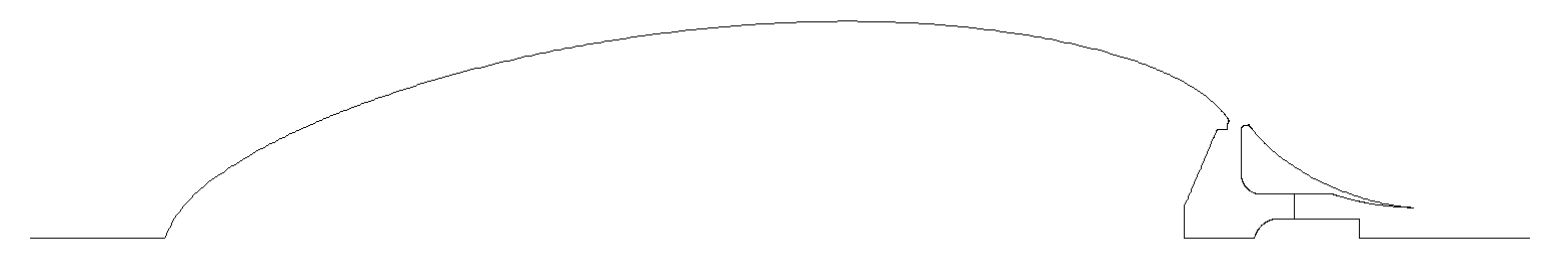

Figure 36: Final body geometry with Cusp A and 0.385 " slot gap

\subsection{Boundary Geometry}

There were three boundary geometries; a freestream "free-air" condition, a straight tunnel, and a tunnel with the contraction modeled. All three were created to test different questions about the body pressure distribution that came up in the initial CFD test runs.

\subsubsection{Freestream Geometry}

The freestream boundary was the first geometry created and it was made for a standard C-grid. It extended 5 chord lengths forward, 5 above, and 10 behind the vehicle. These values were chosen as they represent normal convention for a body that has a relatively small effect on the surrounding flow field. The outline of this can be seen below in Figure 37. 
Figure 37: Freestream geometry

\subsubsection{Straight Tunnel Geometry}

After the initial CFD test cases it was decided to look to see if there was much of a difference between the freestream conditions and tunnel geometry that the tunnel corrections were not capturing. This was determined after looking at the change in $\mathrm{Cp}$ along the body due to buoyancy and other effects. The tunnel was modeled as an axisymmetric tunnel with the same cross sectional area. This was done to maintain the simplicity of the axisymmetric case, but to allow for blockage, buoyancy, and other tunnel effects. The inlet length was sized to give the same boundary layer thickness at the test model as measured in previous tests (see section 3.1.1). This has been done in other CFD cases ${ }^{(16)}$, and is a common practice to give accurate results. The aft section was then carried 10 chord lengths downstream to match the freestream test case. The tunnel boundary can be seen in Figure 38 below.

Figure 38: Straight tunnel geometry 


\subsubsection{Tunnel with Contraction}

Finally after looking at the physical model in the wind tunnel, it was observed that the model was placed so that the nose was at the end of the contraction. It was thought of at one point that, even as the tunnel is straight after that point, the flow still might have some radial velocity that hasn't been fully dissipated. Because of this the tunnel contraction was then modeled. Like the straight tunnel, the contraction was modeled as an axisymmetric model with the same cross sectional area as the real tunnel and the Goldschmied body was placed in the same location as it was in the tests. This can be seen in Figure 39 below.

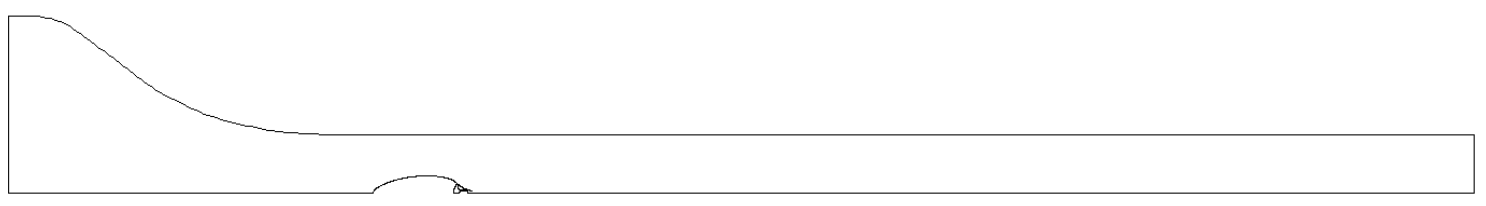

Figure 39: Tunnel with contraction geometry 


\section{Computational Mesh}

The next step in the process going from the geometry file to a solution was to create a computational grid. The governing equations are discretized on this grid, and that process will be discussed in more detail in section 7.1. The mesh is one of the most important parts of computational fluid dynamics and can have a huge effect on the solution if not done correctly. It must break the field up into small enough sections to accurately capture the flow field, but also not too much that it requires too much computational effort to solve.

\subsection{Goldschmied Mesh Generation}

The Goldschmied propulsor had 3 different geometries that were modeled and gridded. It was decided early on that a structured mesh would be used for all the geometries. This was done for a few reasons, one being experience of the author had with structured grid generation versus unstructured generation. It was determined that it was easier to spend a little more effort getting a good structured grid than it was to stumble through the processes of making an unstructured grid.

The structured grid allowed for tighter control of important sections in the model, like the boundary layer, the slot entrance and cusp, and finally the curved streamlines

around the aft section of the body. Also, the computational time needed to solve a structured grid is faster than an unstructured grid. This was not too much of a concern, as the mesh was relatively small and would not make too much difference.

\subsubsection{Body Grid}

The body grid was the first and most important part considered. The grid was held as constant as possible throughout the tunnel geometry changes. This was most important in the aft section where we were the most concerned with the flow and how 
the changes effect the flow there. The largest difference between the tests was that the free stream flow used a structured C-mesh that wrapped around the nose of the body, and the tunnel meshed used an $\mathrm{H}$-mesh to model the flow around the body. These methods were nearly identical to the flow around the body except for the small section in the front. The $\mathrm{C}$-mesh wrapped around the nose, whereas the $\mathrm{H}$-mesh continued down the length of the tunnel. These two meshes can be seen in Figure 40 and Figure 41.

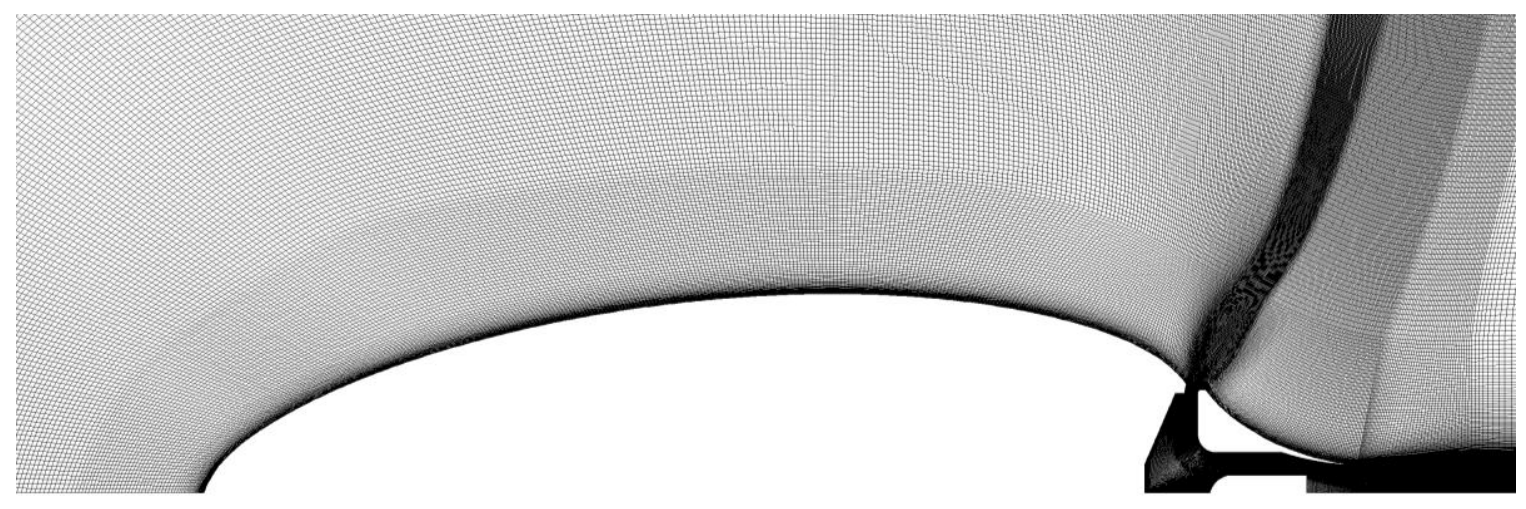

Figure 40: C-mesh around the freestream body geometry

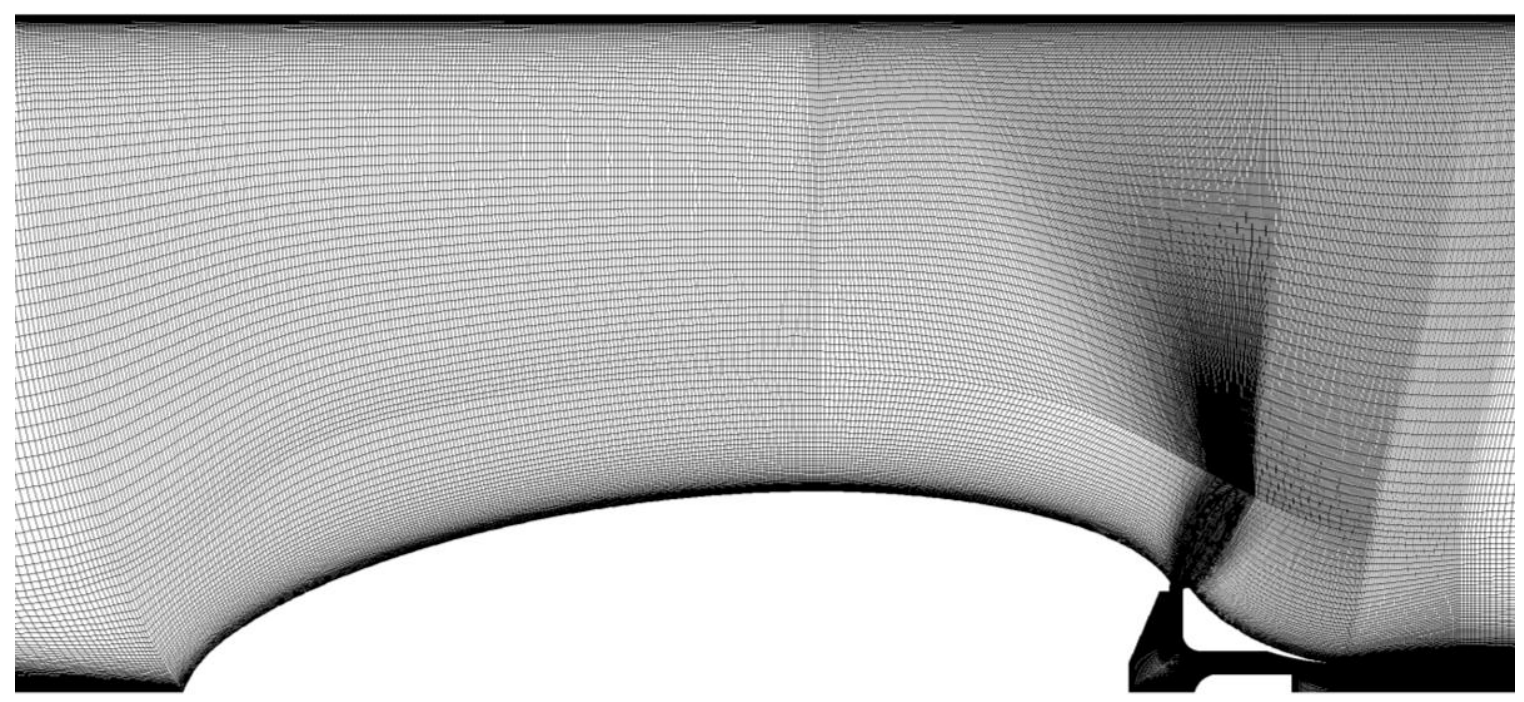

Figure 41: H-mesh around the tunnel body geometry 
When looking at the nose of the body, it was obserbed the C-grid simply wraps around the nose and there are no issues with cells being skewed or stretching the grid around a corner. This was not the case with the $\mathrm{H}$-grid, as it comes down to the nose, it had to manage roughly a $70^{\circ}$ corner to go from the body to horizontally down the tunnel. After an initial mesh, as can be seen in Figure 42, we see that this was easily handed and the cell skewness was not too extreme and was not of concern.

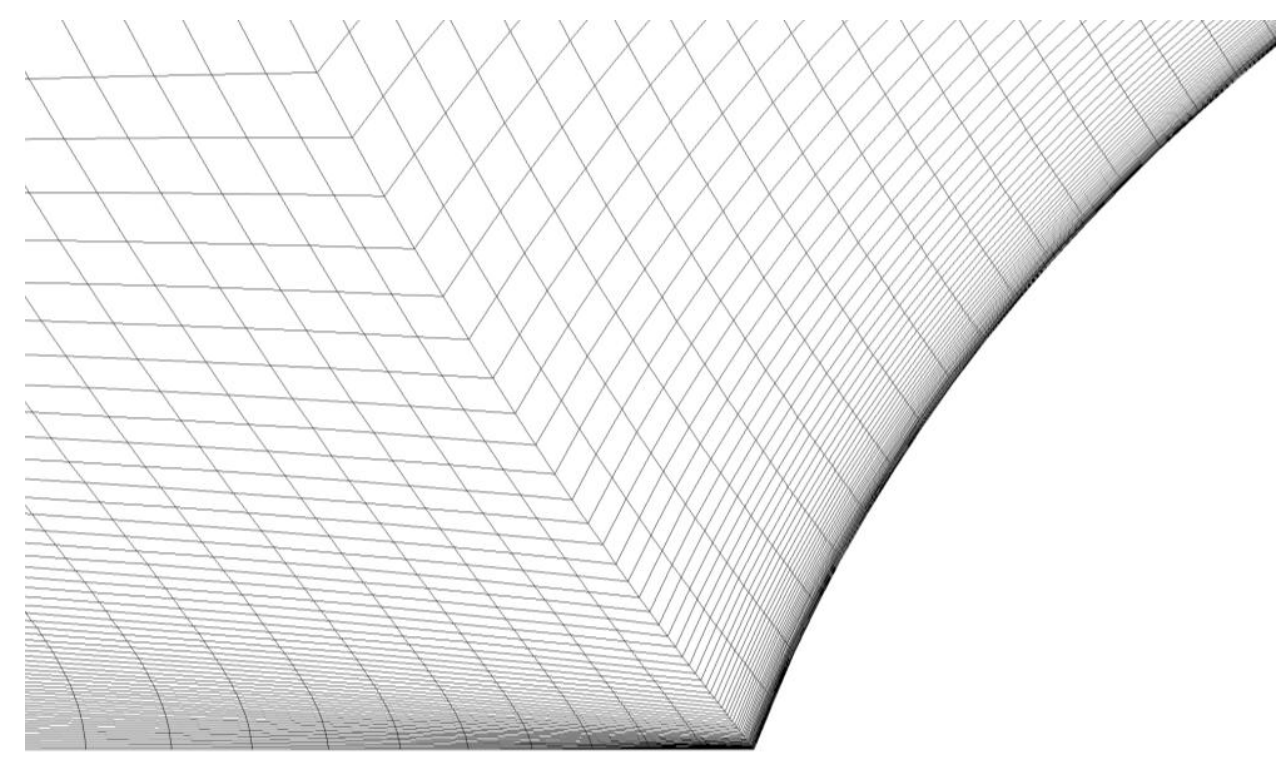

Figure 42: H-grid showing the nose corner meshing

\subsubsection{Aft Section and Internal Slot Meshing}

The next section of concern for modeling was the slot geometry and how that was to be completed. The main grid was run across the slot and then down the aft body where it then left nearly horizontal and continued out to the farfield exit. This approach allowed the grid to closely follow the streamlines when the flow was attached and give the best solutions. 
To model the slot, a section of the structured grid was added to the part that spanned the slot. This was then brought down through the slot, turned as the flow does into the fan, and then brought out the back of the body, parallel to the aft body and continued out horizontally to the farfield exit. This can be seen below in Figure 43.

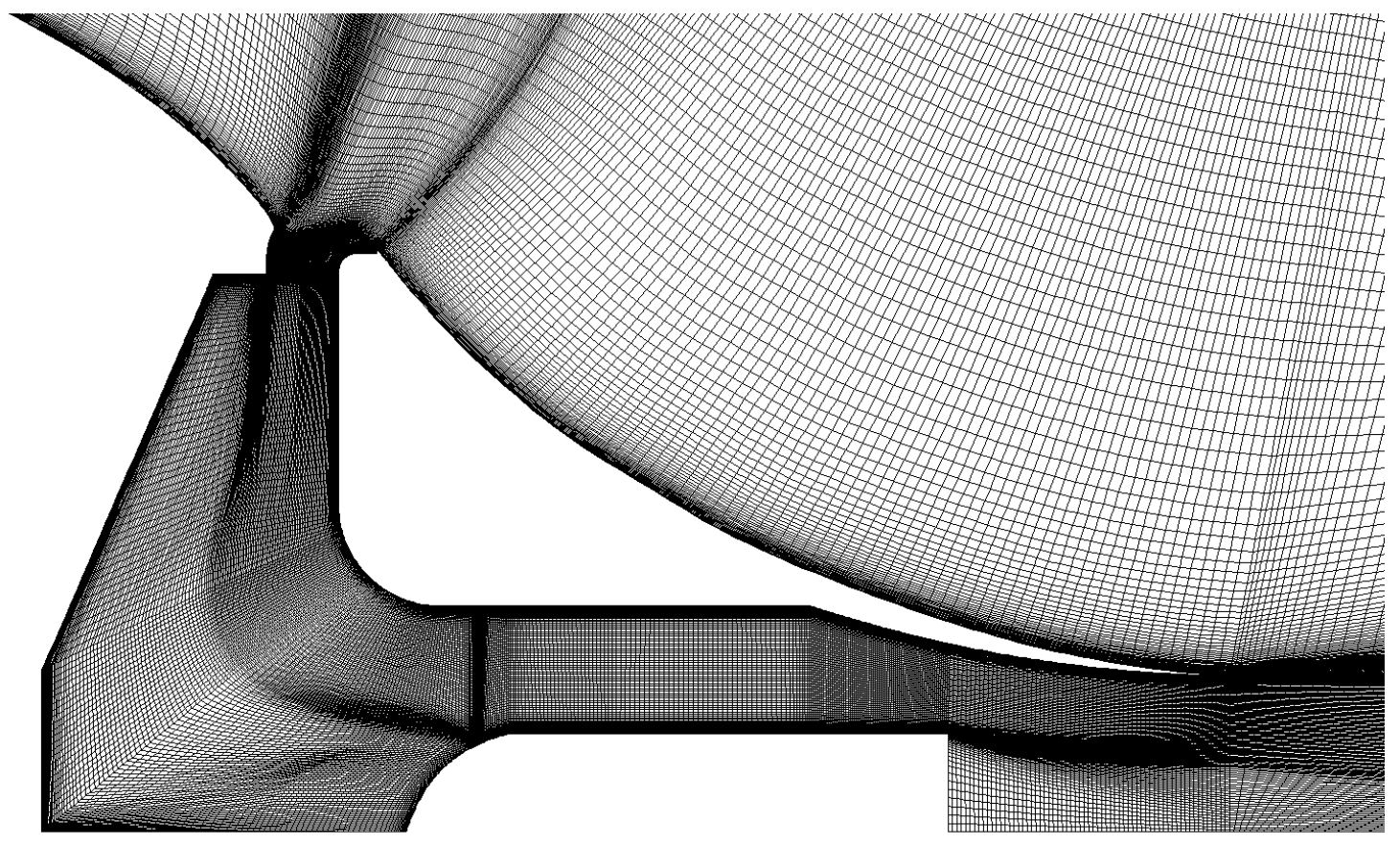

Figure 43: Aft section with slot grid meshing

This was not too difficult to implement and seemed to follow the flow through the slot really well. It also was flexible enough to capture the internal details, like the nose cone for the ducted fan, the step outlet, and most importantly the cusp and inlet geometry, as described in the next section.

\subsubsection{Cusp and Inlet Meshing}

The inlet was the most challenging part of the geometry to accurately capture. After a few trials, it was possible to mesh and make the geometry fit as needed. The geometry can be seen below in Figure 44. 


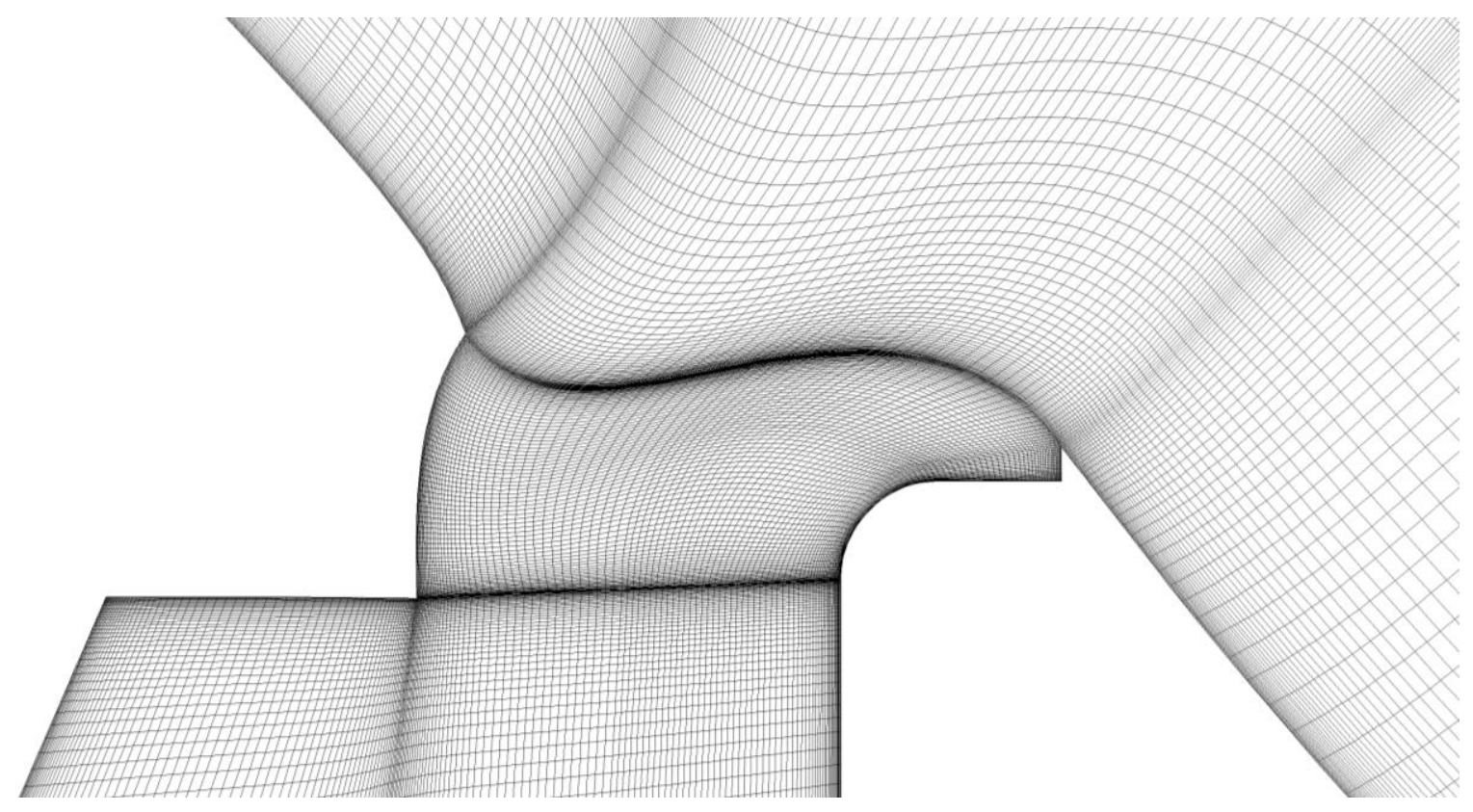

Figure 44: A close view of slot inlet meshing technique

Here the outer grid was snaked to span the gap and continue on the aft section of the model. From there the snaked section was extended further down to capture the lip at the aft section of the slot. This was then rotated and brought down to the slot entrance. This was feasible because the corners were rounded and allowed for the grid to rotate around the corner without any issues.

\subsubsection{Tunnel Modeling}

After the body was modeled, the grid was continued out until it reached the outer boundary walls. For the freestream conditions this was easily done and can be seen in Figure 45. It should be noted that although it looked like an extremely fast cell size change from the near body to the far body, this is not the case. All cell growth in the field was mostly 1.05 and never more than 1.1. The dark regions are because of the lack of resolution of the images. 


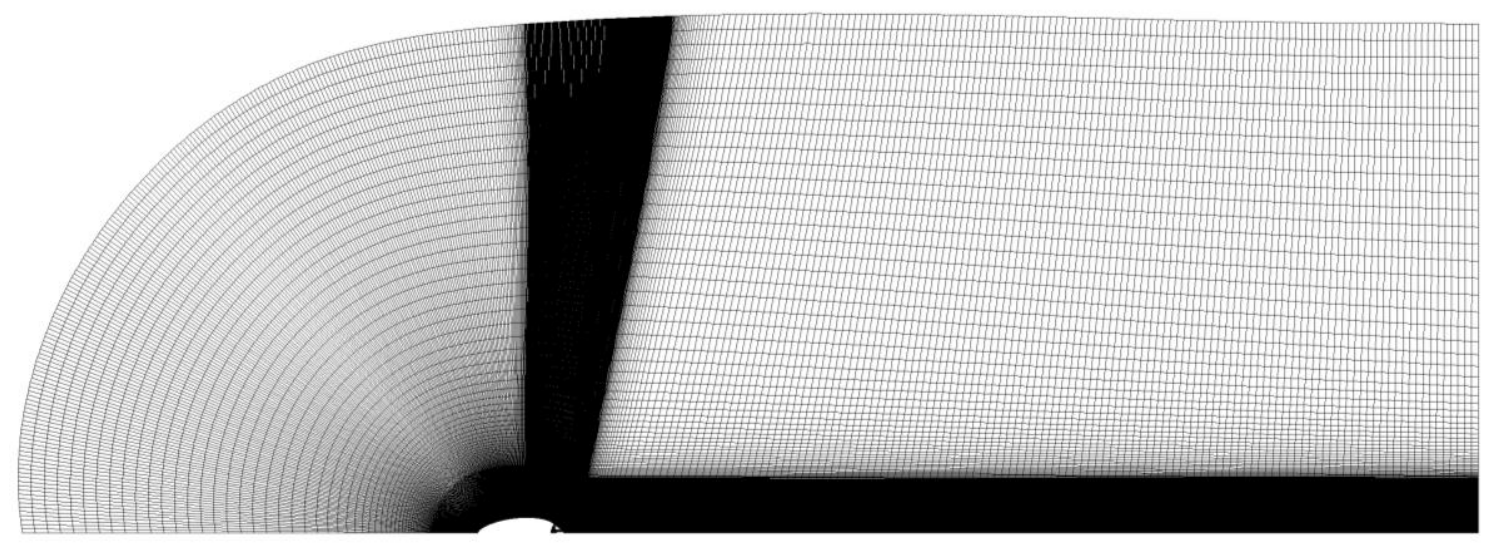

Figure 45: Freestream final mesh

The next piece to be modeled was the straight tunnel section. This was also relatively simple, as the mesh was brought up and then forward and back to the boundary conditions. The only difference here was the wall was modeled on the top section of the mesh. This modeling required the cells to be closely clustered at the wall to catch the viscous effects of the boundary layer. This can be seen in Figure 46.

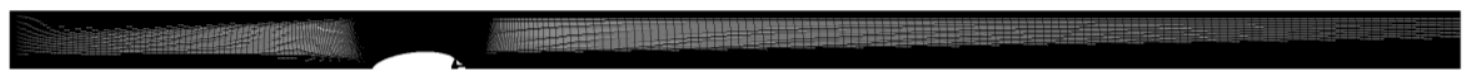

Figure 46: Straight tunnel final mesh

The last case to be modeled was the tunnel with the contraction. As described earlier, this was modeled using the same cross sectional area as the real tunnel with the body placed at the same location. This was similar to the straight tunnel except more consideration was put into the expansion of the grid as it left the nose of the model and continued out to the inlet of the tunnel. The final mesh on the tunnel contraction can be seen in Figure 47 below. The aft section of this mesh was exactly the same as the straight tunnel section. 


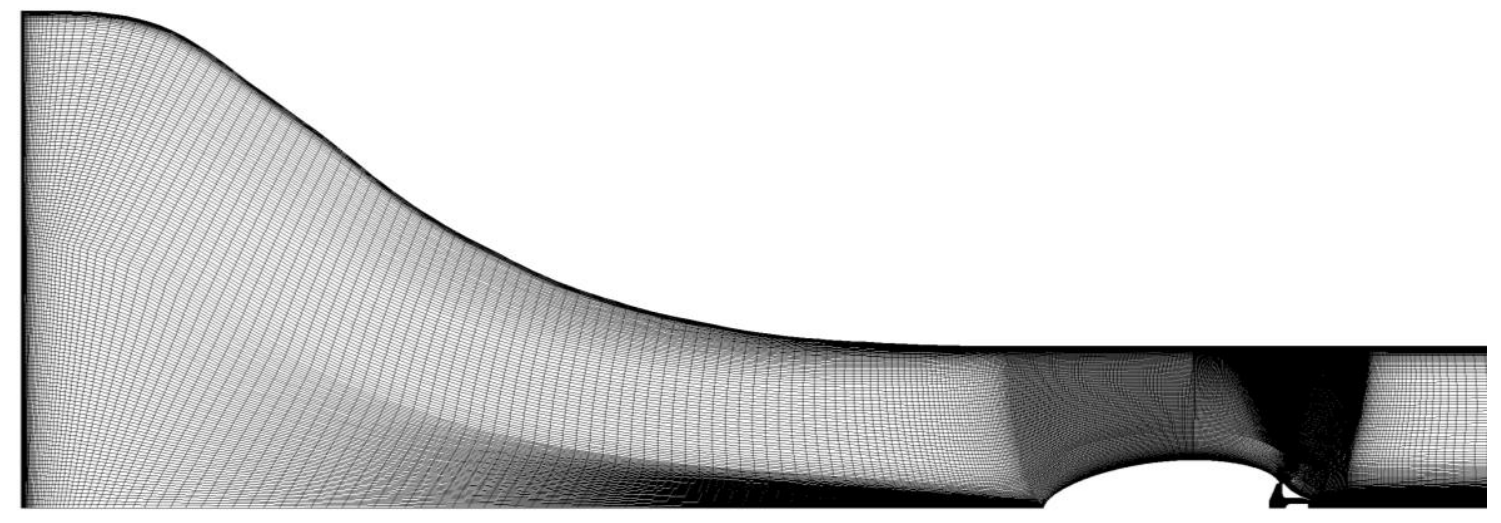

Figure 47: Contraction final mesh

\subsection{Surface Mesh and $Y+$ Calculation}

The surface mesh on the wall surfaces must be small enough to capture the viscous boundary layer effects. This is really important for the turbulence model accuracy and being able to accurately compare the boundary layer. More detail of the needs will be described in the turbulence modeling section, but the main point is that the flow near any solid wall should have a $y+$ value that corresponds to what is needed from the turbulence model. $Y_{+}$is the dimensionless wall distance that is defined as follows ${ }^{(23)}$ :

$$
\boldsymbol{y}^{+} \equiv \frac{u^{*} \boldsymbol{y}}{\boldsymbol{v}}
$$

The $y+$ (from our turbulence modeling) and the kinematic viscosity for the fluid are known. The only value needed is the friction velocity $\left(u^{*}\right)$ to find the needed wall spacing. The friction velocity is defined as:

$$
u^{*} \equiv \sqrt{\frac{\tau_{w}}{\rho}}
$$

And the wall shear stress is defined as:

$$
\tau_{w} \equiv C_{f} * 1 / 2 \rho U_{\infty}^{2}
$$


After determining all of these values, our only unknown was the skin friction value. This could not be computed exactly before knowing the final solution. Because of this the skin friction value was estimated using a skin friction correlation to give us the largest skin friction value that was likely to seen in the flow based on the body length, operating velocity, air density, and dynamic viscosity. There are many methods available to use, for example the $1 / 7^{\text {th }}$ power lay, Schlichting skin-friction formula, Prandtl's formula, and many more. The $y+$ calculator used in our calculations used the Schlichting skin-friction formula. Using the reference values, the wall distances of $1.84 \mathrm{e}-5 \mathrm{~m}$ and $3.07 \mathrm{e}-5 \mathrm{~m}$ would be for $35 \mathrm{~m} / \mathrm{s}$ and $20 \mathrm{~m} / \mathrm{s}$ freestream velocities, respectively. The wall distance required for $35 \mathrm{~m} / \mathrm{s}$ was used in case the $32 \mathrm{~m} / \mathrm{s}$ results were to be compared at a later date.

Results from FLUENT show that for the fan off case, at $20 \mathrm{~m} / \mathrm{s}$, the $y+$ values are always below 1 . For the higher fan speeds the exit velocity is faster than the freestream velocity and here is where we see the increase in $y+$. Although it does increase, the maximum $y+$ is still below a value of 2 , which is still fine, and far below a maximum $y+$ of 5. The plots for fan on and fan off can be seen Figure 48 and Figure 49 below.

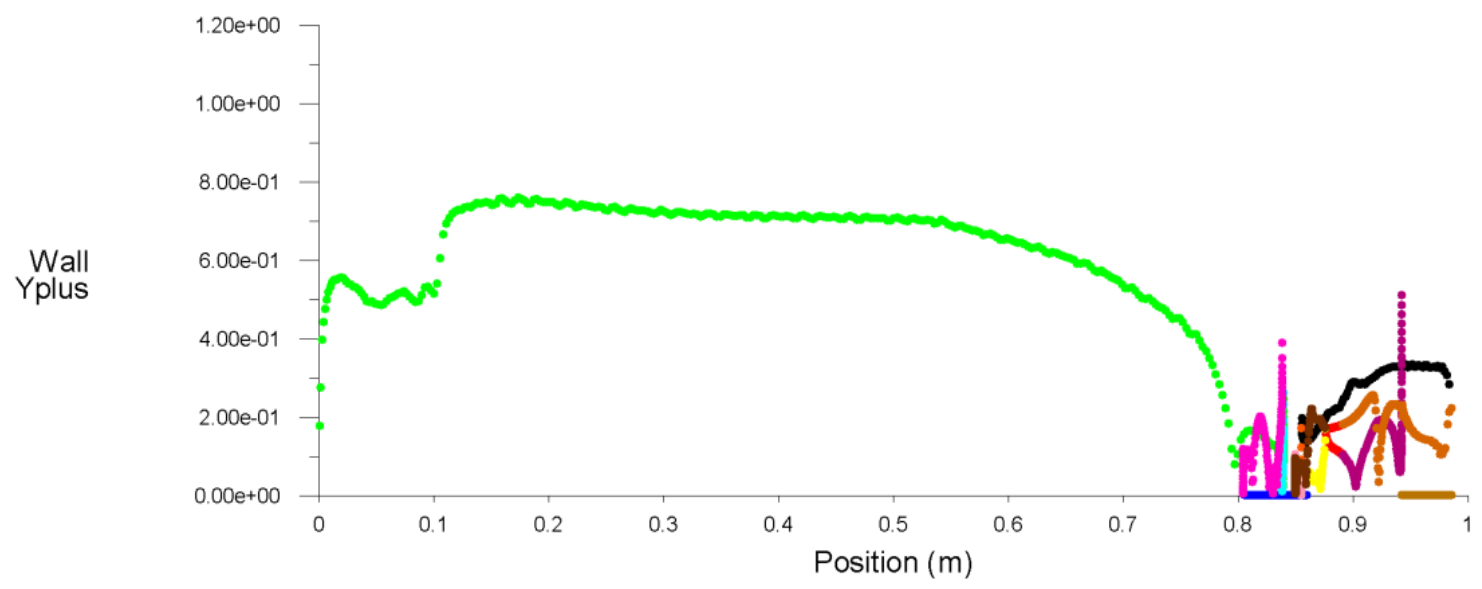

Figure 48: Body $Y+$ values for fan off case 


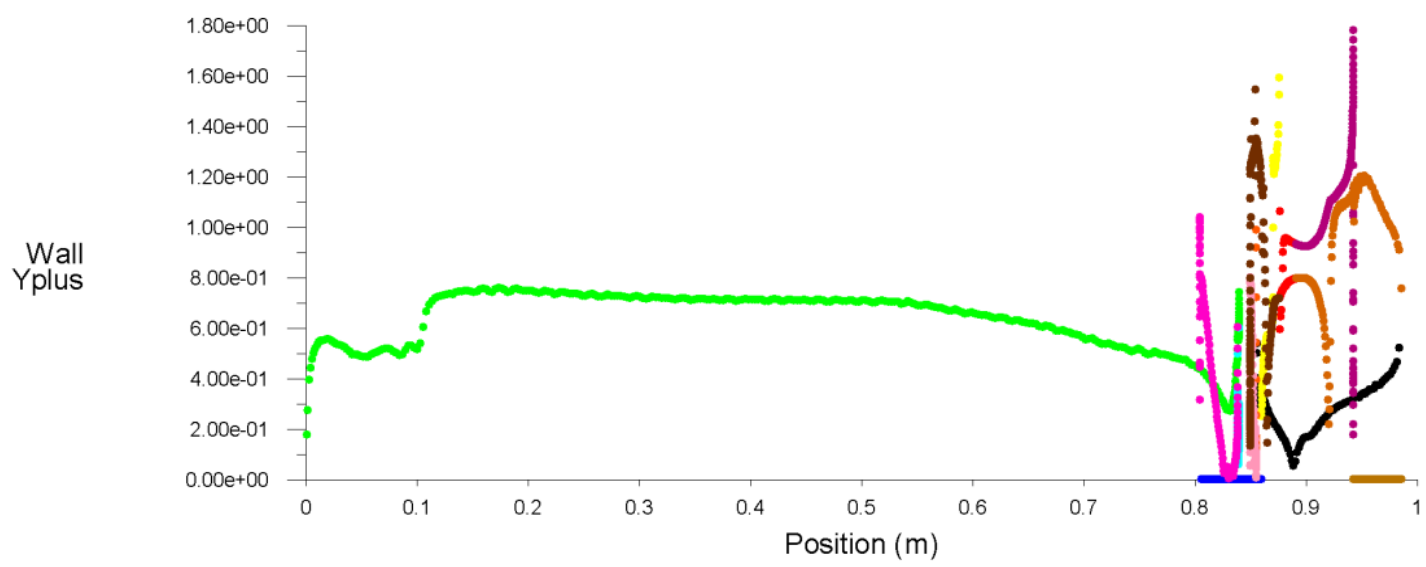

Figure 49: Body $Y+$ values for fan on with a $\triangle P$ of 1500 Pascals

\subsection{Grid Independence Study}

The CFD mesh is one of the single most important items in the whole solution process. Because of its importance, it was good to double check that the flow field has been accurately discretized and that the discretization was on a path to convergence in the asymptotic region. To determine this, it was recommended ${ }^{(24)}$ that CFD applications have a standardized method for finding the discretization error and convergence order. From the wide usage and well tested results, Roache's Grid Convergence Method is the industry standard and will be used in this paper.

\subsubsection{Grid Convergence Index (GCl) Method}

Using Roache's Grid Convergence Method the grid refinement factor ( $r$ ), apparent order $(p)$ are initially found then used to find an exact value along with the error from the finest grid value. The exact value is found by:

$$
\phi_{e x}=\frac{r^{p_{*} \phi_{1}-\phi_{2}}}{r^{p_{-1}}}
$$

And: 


$$
G C I_{\text {fine }}=\frac{F_{s} * e_{a}}{r^{p-1}}
$$

where $\phi_{1}$ and $\phi_{2}$ are the values of interest from the mid and fine grids, $e_{a}$ is the approximate relative error, and $F_{S}$ is the factor of safety.

Oberkampf and Roy ${ }^{(25)}$ suggested that if the apparent order is within $10 \%$ of the formal order then the factor of safety $\left(F_{s}\right)$ should be 1.25 with the apparent order used. If the apparent order is different than the formal order by more than $10 \%$ then a factor of safety $\left(\mathrm{F}_{\mathrm{s}}\right)$ of 3 should be used. Also the exact order should be used in the calculations if the apparent order is greater.

\subsubsection{GCl Results}

The original gird was used with two smaller grids. A small grid refinement factor (1.35) was chosen to keep the grid from getting too coarse. This was used from the recommendation from Celik et al. ${ }^{(24)}$ that from their experience a minimum grid refinement factor of 1.3 or greater be used. This gave grid sizes of 126,086 and 67,234 cells from the original 230,264 . The minimum size of 67,234 did not seem too small, as where in Peraudo's paper ${ }^{(16)}$, the coarsest grids used for the suction slot geometries were 44,032 and 83,140 cells.

Two cases were looked at for the grid independence study, one with the fan off and a second with the fan on. The total pressure drag from the body was used as the critical variable in the study. The three grids were run, for both cases, noting the pressure drag. The fan off case gave an oscillatory convergence, with the middle grid having the smallest drag value of the three grids. Because of this the apparent order was significantly off and the error bars, as seen in the red dashed lines in Figure 50, were relatively large. The $\mathrm{GCl}$ for the fan off grid was approximately $11.0 \%$ for the fine grid value. 


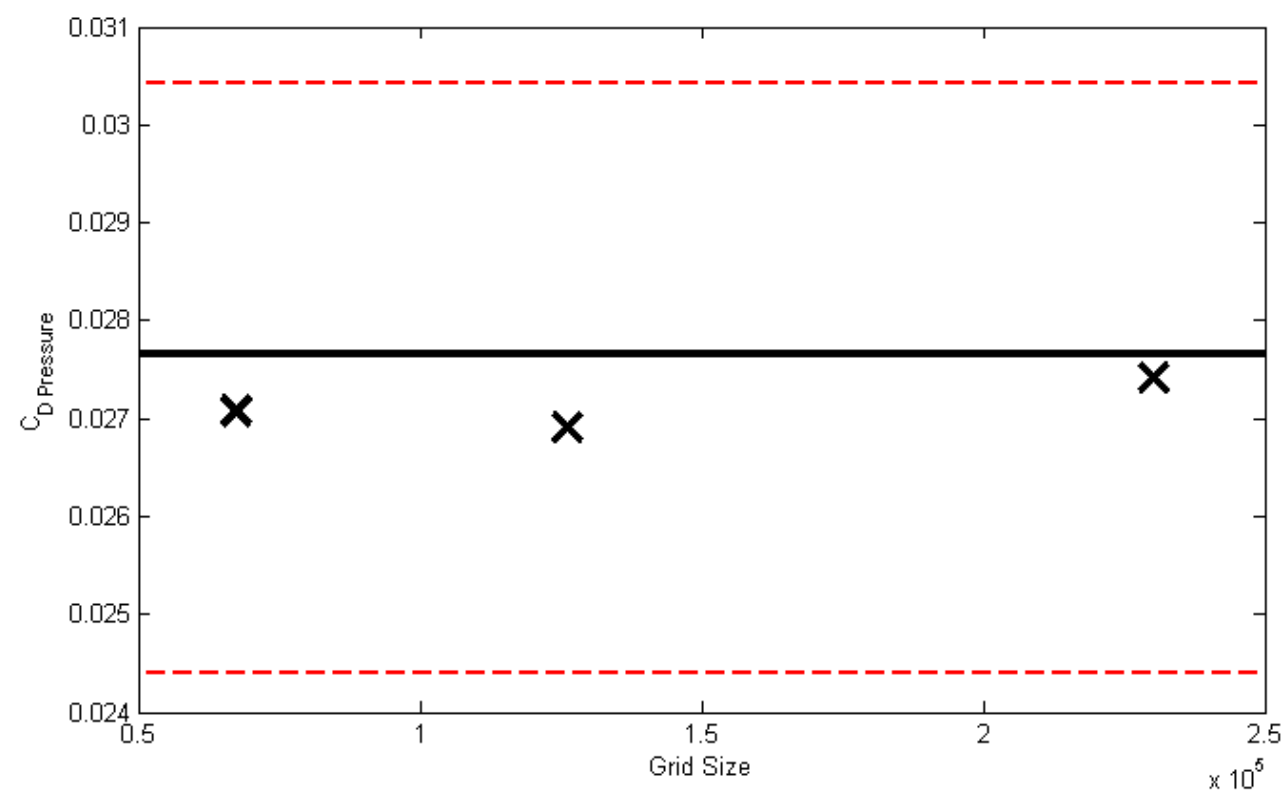

Figure 50: Grid indepence for fan off conditions using the $\mathrm{GCl}$ method

The fan on cases had better performance, with an apparent order of 1.244 compared to the formal order of 1.351 , a difference of $8 \%$. This gave a closer exact value of $C_{D}=0.0272$ and tighter error bars, as the $\mathrm{GCl}$ value was $1.63 \%$ error. The can be seen in Figure 51 below.

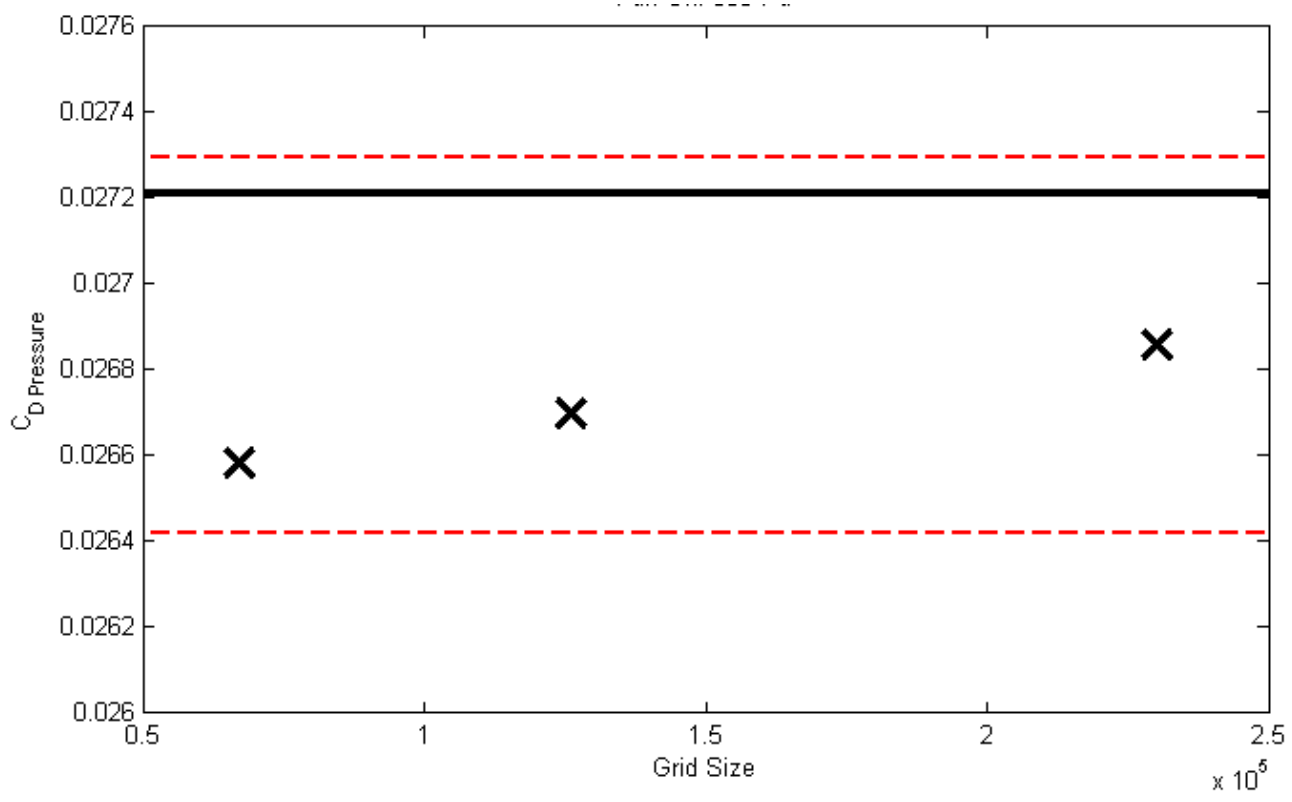

Figure 51: Grid indepence check for fan on (500 pa) conditions using the $\mathrm{GCl}$ method 
The majority of cases ran were with fan on settings, so the $1.63 \%$ error will be used in comparing the different fan on settings. When looking at comparing the fan off settings, the $11.0 \%$ error will be used. 


\section{Computational Method}

ANSYS'S CFD solver, FLUENT, was used for the solver and post processing for all the simulations presented in this paper. The solver used in FLUENT uses the ReynoldsAveraged Navier Stokes equations (RANS) to get a time averaged solution of the flow field.

When solving, the pressure based solver in FLUENT was used. This was chosen because the flow was moving relatively slow (mach $<0.06)$ and this it can be assumed that the flow is incompressible. It makes solving the problem easier, as density does not need to be solved for and that we do not need to solve the energy equation. If it was in the compressible regime, a density based solver would be needed, along with solving the energy equation.

\subsection{Governing Equations}

The governing equations for fluid flows are based on the principles of conservation of mass, momentum, and energy. These equations are made up of coupled, non-linear partial differential equations that have no general exact solutions; rather they have to be solved numerically through a highly iterative process. The derivations of these equations

are beyond the scope of this paper and are in most modern aerodynamic textbooks ${ }^{(23)(26)}$ and classes. What is presented here is based on the description that FLUENT presents in its theory guide. ${ }^{(27)}$

\subsubsection{Continuity Equation}

FLUENT has the conservation of mass written as:

$$
\frac{\partial \rho}{\partial t}+\nabla \cdot(\rho \vec{v})=S_{m}
$$


where the additional $S_{m}$ term account for phase change, like in evaporation, or for any user-defined sources. For most cases this is set to zero and we then get the standard continuity equation. Because our cases are 2D axisymmetric, we would like to note that the continuity equation is changed into polar coordinates; $x$ and $r$

$$
\frac{\partial \rho}{\partial t}+\frac{\partial}{\partial x}\left(\rho v_{x}\right)+\frac{\partial}{\partial r}\left(\rho v_{r}\right)+\frac{\rho v_{r}}{r}=S_{m}
$$

\subsubsection{Momentum Equation}

Conservation of momentum in a non-accelerating inertial reference frame can be described as

$$
\frac{\partial}{\partial t}(\rho \vec{v})+\nabla \cdot(\rho \vec{v} \vec{v})=-\nabla \mathbf{p}+\nabla \cdot(\overline{\vec{\tau}})+\rho \vec{g}+\vec{F}
$$

where $\mathrm{p}$ is the static pressure, $\rho \vec{g}$ and $\vec{F}$ are the gravitational and external forces, and finally $\overline{\bar{\tau}}$ is the stress tensor. Which can be given by

$$
\overline{\vec{\tau}}=\mu\left[\left(\nabla \vec{v}+\nabla \vec{v}^{T}\right)-\frac{2}{3} \nabla \cdot \vec{v} I\right]
$$

where $\mu$ is the molecular viscosity, $I$ is the unit tensor.

The momentum equation is modified slightly for $2 \mathrm{D}$ axisymmetric cases, the axial and radial equations are given below.

$$
\begin{aligned}
& \frac{\partial}{\partial t}\left(\rho v_{x}\right)+\frac{1}{r} \frac{\partial}{\partial x}\left(r \rho v_{x} v_{x}\right)+\frac{1}{r} \frac{\partial}{\partial r}\left(r \rho v_{r} v_{x}\right)=-\frac{\partial p}{\partial x}+\frac{1}{r} \frac{\partial}{\partial x}\left[r \mu\left(2 \frac{\partial v_{x}}{\partial x}-\frac{2}{3}(\nabla \cdot \vec{v})\right]\right. \\
& +\frac{1}{r} \frac{\partial}{\partial x}\left[\boldsymbol{r} \mu\left(2 \frac{\partial v_{x}}{\partial x}+\frac{\partial v_{r}}{\partial x}\right)\right]+F_{x}
\end{aligned}
$$

and

$\frac{\partial}{\partial t}\left(\rho v_{r}\right)+\frac{1}{r} \frac{\partial}{\partial x}\left(r \rho v_{x} v_{r}\right)+\frac{1}{r} \frac{\partial}{\partial r}\left(r \rho v_{r} v_{r}\right)=-\frac{\partial p}{\partial x}+\frac{1}{r} \frac{\partial}{\partial x}\left[r \mu\left(\frac{\partial v_{r}}{\partial x}-\frac{\partial v_{x}}{\partial r}\right)\right]$ 


$$
+\frac{1}{r} \frac{\partial}{\partial r}\left[r \mu\left(2 \frac{\partial v_{r}}{\partial x}-\frac{2}{3}(\nabla \cdot \vec{v})\right)\right]-2 \mu \frac{v_{r}}{r^{2}}+\frac{2}{3} \frac{\mu}{r}(\nabla \cdot \vec{v})+\rho \frac{v_{z}^{2}}{r}+F_{x}
$$

where

$$
\nabla \cdot \vec{v}=\frac{\partial v_{x}}{\partial x}+\frac{\partial v_{r}}{\partial r}+\frac{v_{r}}{r}
$$

\subsubsection{Energy Equation}

The energy equation can be written as

$$
\frac{\partial}{\partial t}(\rho E)+\nabla \cdot[\vec{v}(\rho E+p)]=\nabla \cdot\left[k_{e f f} \nabla T-\sum h_{j} \overrightarrow{J_{j}}+\left(\overline{\overline{\tau_{e f f}}} \cdot \vec{v}\right)\right]+S_{h}
$$

the three bracketed terms on the right hand side of the equation are the energy transfer due to conduction, species diffusion, and viscous dissipation.

We have included the energy for good measure but it was not used. The energy equation did not need to be solved as we used a pressure based solver, no heat transfer was assumed, and that heat generation due to viscous effects was negligible. Also our flow was quite slow ( mach 0.06$)$ so no compressibility effects are seen and the density can be assumed to be constant.

\subsection{Turbulence Modeling}

When using the Reynolds-Averaged Navier Stokes equations, there is one term in the momentum equation, the Turbulent-Shear also known as the Reynolds-Stress Term: $\left(-\rho \overline{u^{\prime} v^{\prime}}\right)$, that cannot be calculated directly from known values. Because we are not able to calculate the turbulent term directly, turbulence models are used to approximate these

values. The following sections show the process of modeling this turbulence term, highlighting the turbulence models that FLUENT uses. 
It should be noted that direct numerical simulation (DNS) can calculate these values, but it is highly computationally expensive. Because of this DNS is only used on geometric simple problems and low Reynolds number flows.

\subsubsection{Boussinesq Hypothesis}

The Boussinesq hypothesis is used in turbulence models like the SparlartAllmaras, $k-\varepsilon$, and $k-\omega$ models and is how these models relate the Reynolds stresses to the mean velocity gradients. Here it assumes that the turbulent viscosity is an isotropic scalar quantity, which isn't exactly true but is a good approximation for most flows. The form of the Boussinesq approximation that FLUENT uses is shown below:

$$
\left(-\rho \overline{u_{\imath}{ }^{\prime} v_{J}^{\prime}}\right)=\mu_{t}\left(\frac{\partial \overline{u_{l}}}{\partial x_{j}}+\frac{\partial \overline{u_{J}}}{\partial x_{i}}\right)-\frac{2}{3}\left(\rho k-\mu_{t} \frac{\partial \overline{u_{k}}}{\partial x_{k}}\right) \delta_{i j}
$$

All of these terms are known except the turbulent viscosity, $\mu_{t}$, this is the term to be computed by the first three turbulence models described below. Once the turbulent viscosity is found, it is plugged into the Boussineq approximation and the Reynolds stresses are found.

Higher order solutions, like the transition and Reynolds stress models, do not use

the Boussinesq approximation. They do not assume that the turbulent viscosity is isotropic, which plays a role in highly swirling flows and stress-driven secondary flows. With this detail additional equations are needed to compute the flow, five for $2 \mathrm{D}$ and seven more for 3D flows.

\subsubsection{Sparlart - Allmaras}

The Sparlart-Allmaras is the simplest model offered by FLUENT that takes into account turbulent flows (as opposed to inviscid and laminar models). The SparlartAllmaras model is a one equation model, which means it used one more partial 
differential equation to solve for the Reynolds stress term. It is a turbulence model that was developed for the aerospace industry and has shown good results for boundary layers subjected to adverse pressure gradients. While good at boundary layer flows, the Sparlart-Allmaras model has shortcomings with general industrial flows, like free shear flows including plane and round jets. With these cases it produces relatively large error.

The Sparlart-Allmaras model uses the Boussinesq approximation and in doing so, it relates the transport variable $\widetilde{v}$ to the turbulent viscosity by the following equation

$$
\mu_{t}=\rho \tilde{v} f_{v 1}
$$

where $f_{v 1}$ is the viscous damping function. The transport variable is defined by the differential transport equation:

$$
\frac{\partial}{\partial t}(\rho \widetilde{\mathcal{v}})+\frac{\partial}{\partial x_{i}}\left(\rho \widetilde{\mathcal{v}} u_{i}\right)=G_{v}+\frac{1}{\sigma_{\widetilde{v}}}\left[\frac{\partial}{\partial x_{j}}\left\{(\mu+\rho \widetilde{\mathcal{v}}) \frac{\partial \widetilde{v}}{\partial x_{j}}\right\}+C_{b 2} \rho\left(\frac{\partial \widetilde{v}}{\partial x_{j}}\right)^{2}\right]-Y_{v}+S_{\widetilde{v}}(16)
$$

where $G_{v}$ represents the production of turbulence viscosity, $Y_{v}$ is the destruction and $S_{\widetilde{v}}$ is the user-defined source term. More details about the intricacies and implementation can be found in the FLUENT theory guide ${ }^{(27)}$, FLUENT user's guide ${ }^{(28)}$, and CFD textbooks ${ }^{(23)(26)(29)}$.

\subsection{3 $\mathrm{k}-\varepsilon$ Model}

The standard $\mathrm{k}-\varepsilon$ model is a model based on model transport equations for the turbulence kinetic energy $(k)$ and its dissipation rate $(\varepsilon)$. Like the Sparlart-Allmaras model, these two variables are used to compute the turbulent viscosity from the Boussinesq approximation.

$$
\mu_{t}=\rho C_{\mu} \frac{k^{2}}{\varepsilon}
$$

where $C_{\mu}$ is a constant. 
The turbulent kinetic energy resembles equations derived from the exact transport equation, whereas the dissipation equation was made from physical reasoning and is not linked to its mathematical counterpart. The standard $k-\varepsilon$ model can be seen below:

$$
\frac{\partial}{\partial t}(\rho k)+\frac{\partial}{\partial x_{i}}\left(\rho k u_{i}\right)=\frac{\partial}{\partial x_{j}}\left[\left(\mu+\frac{\mu_{t}}{\sigma_{k}}\right) \frac{\partial k}{\partial x_{j}}\right]+G_{k}+G_{b}-\rho \varepsilon-Y_{M}+S_{k}
$$

and

$$
\begin{aligned}
\frac{\partial}{\partial t}(\rho \varepsilon)+\frac{\partial}{\partial x_{i}}\left(\rho \varepsilon u_{i}\right) & =\frac{\partial}{\partial x_{j}}\left[\left(\mu+\frac{\mu_{t}}{\sigma_{\varepsilon}}\right) \frac{\partial \varepsilon}{\partial x_{j}}\right] \\
& +C_{1 \varepsilon} * \frac{\varepsilon}{k}\left(G_{k}+C_{3 \varepsilon} G_{b}\right)-C_{2 \varepsilon} \rho \frac{\varepsilon^{2}}{k}+S_{k}
\end{aligned}
$$

where $G_{k}$ is the generation of turbulent kinetic energy due to velocity gradients, $G_{b}$ is generation due to buoyancy kinetic energy, and $Y_{M}$ is the contribution of fluctuating dilatation in compressible turbulence. The standard $k-\varepsilon$ model has weakness that has led to modifications to improve the model. These modifications improve the model in most every aspect, and because of this the standard version is rarely used. Rather FLUENT has both a RNG $\mathrm{k}-\varepsilon$ and Realizable $\mathrm{k}-\varepsilon$ model.

The "renormalization group" RNG $k-\varepsilon$ has additional terms to improve the accuracy for rapidly strained flows, to include the effects of swirl, self-calculated Prandtl numbers, and low Reynolds number. From these improvements the RNG $\mathrm{k}-\varepsilon$ model outperforms the standard model and is applicable for a wider class of flows.

Similarly the realizable $\mathrm{k}-\varepsilon$ improves on the standard $\mathrm{k}-\varepsilon$ model by containing an alternative formulation for the turbulent viscosity and a modified transport equation for the dissipation rate. The modified transport equation was derived from an exact equation 
for the vorticity fluctuation. Both the RNG $k-\varepsilon$ and Realizable $k-\varepsilon$ have considerable improvements over the standard $k-\varepsilon$ model. It is not clear which of the two are better, but some initial studies have shown that the Realizable $k-\varepsilon$ performs better for separated and complex flows.

\subsection{4 k - $\omega$ Model}

The standard $k-\omega$ model is an empirical model based on model transport equations for the turbulence kinetic energy $(k)$ and the specific dissipation rate $(\omega)$. Similarly to the $k-\varepsilon$ model the two variables are used to compute the turbulent viscosity from the Boussinesq approximation.

$$
\mu_{t}=\alpha^{*} \frac{\rho k}{\omega}
$$

The turbulent kinetic energy and specific dissipation rate are found using the following transport equations:

$$
\frac{\partial}{\partial t}(\rho k)+\frac{\partial}{\partial x_{i}}\left(\rho k u_{i}\right)=\frac{\partial}{\partial x_{j}}\left[\Gamma_{k} \frac{\partial k}{\partial x_{j}}\right]+G_{k}-Y_{k}+S_{k}
$$

and

$$
\frac{\partial}{\partial t}(\rho \omega)+\frac{\partial}{\partial x_{i}}\left(\rho \omega u_{i}\right)=\frac{\partial}{\partial x_{j}}\left[\Gamma_{\omega} \frac{\partial \omega}{\partial x_{j}}\right]+G_{\omega}-Y_{\omega}+S_{\omega}
$$

where $G_{k}$ represents the generation of turbulent kinetic energy due to velocity gradients and $G_{\omega}$ represents the generation of the specific dissipation rate; $Y_{k}$ and $Y_{\omega}$ are the dissipation of these values. $\Gamma_{k}$ and $\Gamma_{\omega}$ are the effective diffusivity for $k$ and $\omega$, respectively. The standard $k-\omega$ model also has weaknesses that have led to its modification. FLUENT uses the Shear Stress Transport (SST) $k-\omega$ model as the modified $\mathrm{k}-\omega$ model. 
The SST $k-\omega$ model improves on the standard $k-\omega$ model by creating a gradual change from the standard version in the inner region of the boundary layer to a high Reynolds number version of the $\mathrm{k}-\varepsilon$ model in the outer region of the boundary layer. It also includes a modified turbulent viscosity formulation to account for shear stress transport effects. These modifications make the SST $k-\omega$ model more accurate and reliable for more flows, including adverse pressure gradients, airfoils, transonic shock waves and more. Because of its ability to predict separation and adverse pressure gradients, the SST $k-\omega$ model was chosen as the initial model and the other turbulence models tested will be compared to it.

\subsubsection{Transition $\mathbf{k}-\mathbf{k l}-\boldsymbol{\omega}$}

From FLUENT's theory guide ${ }^{(27)}$ : “The $\mathrm{k}-\mathrm{kl}-\omega$ transition model is used to predict boundary layer development and calculate transition onset. This model can be used to effectively address the transition of the boundary layer from a laminar to turbulent regime." For all of Roepke and Thomason's testing the model had transition strips located at $10 \%$ model length. After the flow was tripped, it remained turbulent the rest of the body length, as verified by boundary layer stethoscope listening. Because of this, for all CFD runs the flow was taken as turbulent over the whole body, as the first 4 inches of laminar flow was not seen as important as focusing on the separation on the aft section. This led to the transition model not being need.

\subsubsection{Transition Shear Stress Transport}

The transition SST model is based on the SST $k-\omega$ model with the addition of two more partial differential equations to estimate the location of transition between laminar and turbulent flow, to make it a 4-equation model. Similarly to the $k-k l-\omega$ transition model, the transition SST model was deemed un-necessary. 


\subsubsection{Reynolds Stress}

The Reynolds stress model (RSM) is the most complicated model FLUENT offers, as it is a 5-equation model. It no longer uses the Boussinesq approximation; it assumes an anisotropic eddy-viscosity. The RSM better accounts for complex flows like high streamline curvature, swirl, rotation, and rapid changes in strain rate than the zero, one, and two equation models.

In the RSM the Reynolds stress is calculated through an expanded form that relates the local time derivative and convection of the Reynolds stress to the turbulent diffusion, molecular diffusion, stress production, buoyancy production, pressure strain, dissipation, production by system rotation, and a user-defined source term. For more details and implementation, the reader is directed to the FLUENT theory guide ${ }^{(27)}$.

\subsubsection{Wall Treatment}

As said before the grid was created to give a $y+$ of less than 1 most places and less than 2 everywhere. This allowed us to calculate the turbulence through the boundary layer and we did not have to use any wall functions, the turbulence models are valid through the near all region. If a $y+$ of less than 5 was not implemented then a $y+$ between 30 and 150 could be used in combination with wall functions. FLUENT's theory guide shows the difference between the two methods, as seen in Figure 52. 


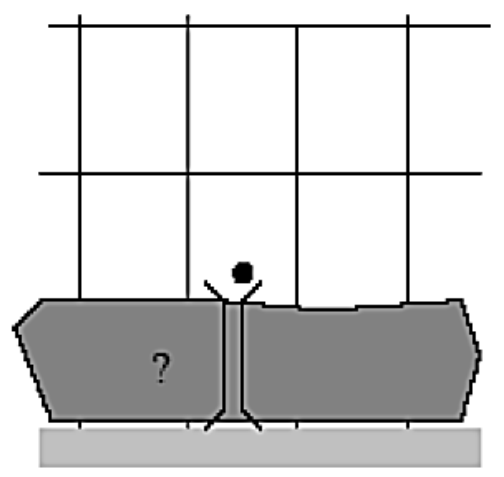

Wall Function Approach

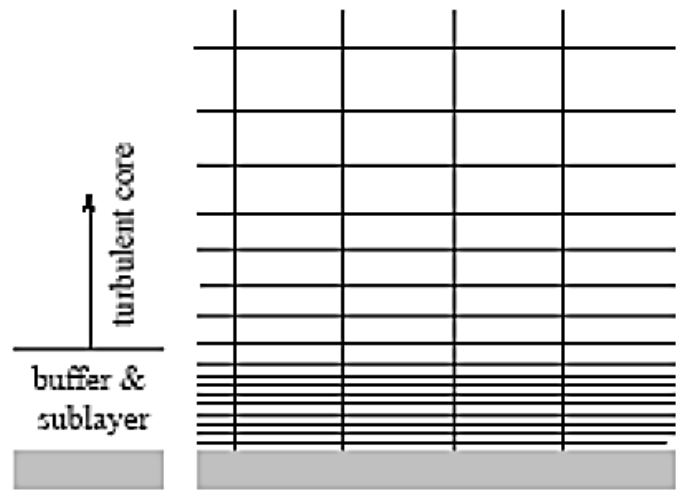

Near-Wall Model Approach

Figure 52: Visual comparison of near wall treatment methods ${ }^{(27)}$

These sizes are based on trying to get the cell nearest to the wall to fall in either the viscous sublayer region, for the near-wall approach, or the fully turbulent log-law region, for the wall functions. The different near-wall regions can be seen in Figure 53 below.

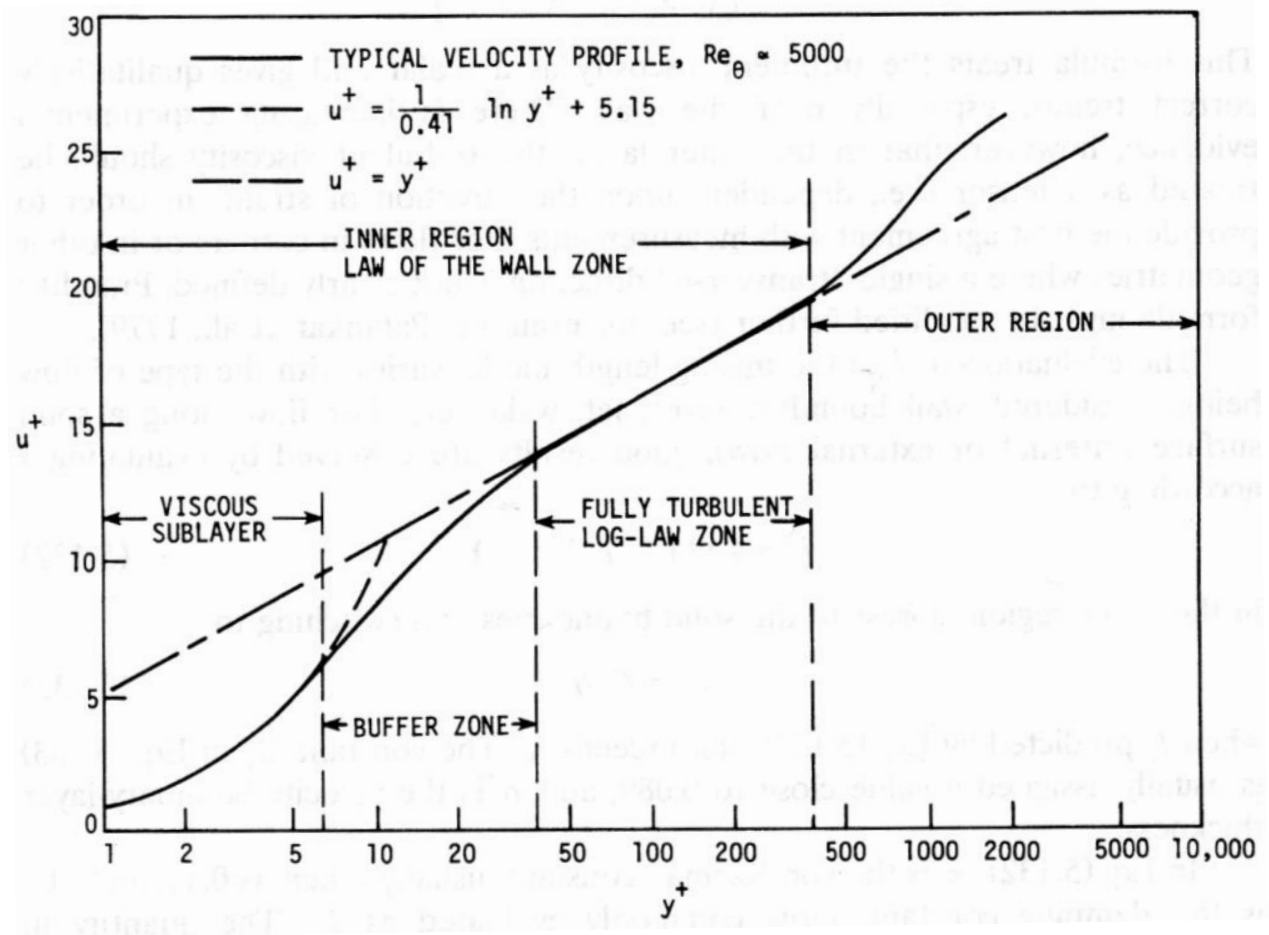

Figure 53: Zones in a typical incompressible turbulent boundary layer ${ }^{(26)}$ 


\subsection{Operating Conditions}

All meshing, computing, and post processing was done with ANSYS's 12.1 software suite. The Mesh was generated in ICEM and then brought over to FLUENT. Once in FLUENT the boundary conditions were set up, the flow as then initialized and solve, and finally the post processing was also done in FLUENT.

\subsubsection{Boundary Conditions}

The boundary conditions were set up for the pressure based solver. The inlet, the front face for the tunnel grids and the front/top side of the C-grids, were specified as velocity inlets. The speed was matched to the wind tunnel data, with the inlet at $21.4 \mathrm{~m} / \mathrm{s}$ parallel to the axis and a speed of $2.25 \mathrm{~m} / \mathrm{s}$ was used in the tunnel contraction geometry to get the $21.4 \mathrm{~m} / \mathrm{s}$ after the contraction. This was based on the $9.42: 1$ tunnel contraction. The turbulence was also specified at the inlet, from the Flow Characteristics in the Renovated 3x4ft Wind Tunnel paper ${ }^{(20)}$, we used the turbulence intensity of $0.5 \%$ and a mixing length of $0.08 \mathrm{~m}$. The mixing length was determined from mixing length $=$ $0.07^{*}$ hydraulic diameter, which is our tunnel diameter, giving us the value of $0.08 \mathrm{~m}$. The outlet was modeled as an outflow condition, which in FLUENT, meant zero diffusion flux for all flow variables and an overall mass balance correction. The fluxes were zero in the plane normal to the exit boundary and they simulated a fully developed flow that had no influence in the upstream direction. The bottom sections of the $2 \mathrm{D}$ geometry were defined as the axis boundary. Here the rotation was assumed around the x-axis with the origin at $(0,0)$.

The body used no-slip wall conditions with the default values for wall roughness height and other constants. There was no thermal, radiation, slip or any other 
specifications enabled. The final boundary condition was for the fan in the aft section of the body. In Cal Poly's wind tunnel model there were pressure ports fore and aft of the fan blades, which measured pressure rise across the fan disk. This was modeled as a single plane in FLUENT and the pressure rise was modeled as a pressure jump across that boundary. The pressure jump across the fan can be seen in Figure 54 below.

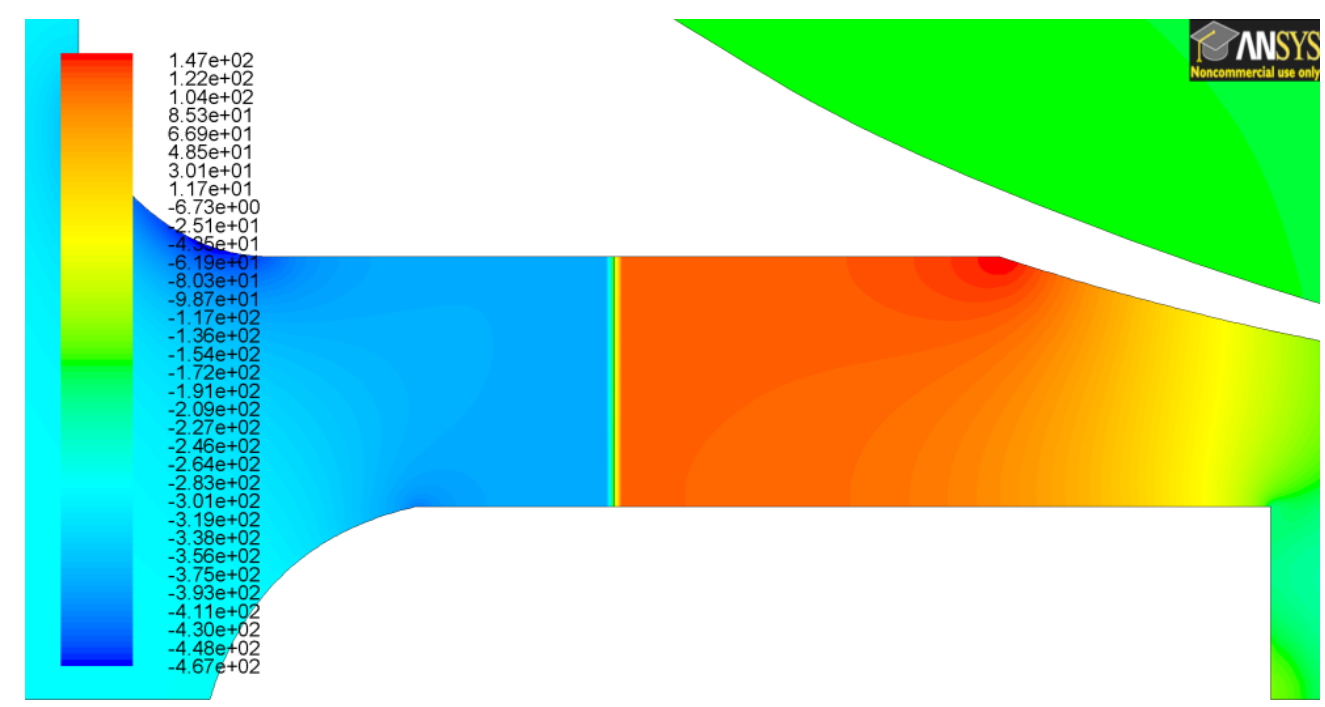

Figure 54: Contours of static pressure across the fan with a pressure increase of 500 pascals

\subsubsection{Solver Conditions}

FLUENT was run using a steady axisymmetric pressure-based solver with absolute velocity formulation. The working fluid was FLUENT's 'air' set as a constant density of $1.225 \mathrm{~kg} / \mathrm{m}^{3}$ and constant viscosity of $1.7894 \mathrm{e}-5 \mathrm{~kg} / \mathrm{m}-\mathrm{s}$. The SIMPLE scheme was used for pressure-velocity coupling. The gradient used the least squares cell based, the pressure used second order, and the momentum, turbulent kinetic energy and specific dissipation rate all used third order MUSCL schemes. To start off the under- 
relaxation factors were brought down a little, then after the flow initialized, the underrelaxation values were brought back to their default values.

\subsubsection{Convergence Criteria}

Three main convergence criteria were examined to test for convergence of the model. First, the drag coefficient was checked to determine whether or not it has stabilized. The flow was pretty well behaved and the drag coefficient would oscillate for a few hundred iterations but then would dampen out pretty quickly to a single value. Next, the mass flow rates at different stations throughout the slot were examined. When the fan speed or other geometry was changed, these numbers no longer matched. This meant that there was a mass imbalance within the slot and the solution needed more iterations until a steady state was reached. The final item that was looked at was that the changes have propagated far enough downstream that it no longer affected the body. This was seen visually as what seemed like a wave propagated downstream as it iterated. This was done because for small changes the flow around the body converged faster than the wake did.

These three convergence criteria were examined looked for every case, but for trials where multiple different settings were being tested, like fan speeds, a set number of iterations were done for all settings to insure that all flows were converged to a comparable degree. This was done for both simplicity and uniformity in case there was something small that was sill changing but was not noticeable to any of the three convergence criteria. 


\subsection{Computational Resources}

The 2D axisymmetric cases were small enough to be run on available computers overnight. The flight lab on campus was equipped with Dell Optiplex 755's that had an Intel Core 2 Quad CPU Q9450 with both processors clocked at 2.67GHz. They were running Windows 7 64-bit with 4GB of RAM. These had enough space and power to run the jobs overnight. Six computers were each set up with the ANSYS suite and each one was able to run an independent job at the same time. 


\section{Results}

We will first present the results from the CFD trials here, outlining the trends that are found with the different operating conditions. Then in section 0 we will compare the CFD results to Roepke and Thomason's tunnel results.

\subsection{Initial Testing and Observations}

In the initial grid generation and solving, there were a number of initial test cases that were used to both understand the software being run and the geometry that had to be modeled. The ANSYS software suite had not been used previously and a lot of effort in the beginning of the project was centered on learning the intricacies of the software. Training began with using the tutorials for $2 \mathrm{D}$ meshing and solving in FLUENT, then progressed to looking through the user manuals and theory guides to figure out what settings could be modified and adjusted. Some confusion early in the process stemmed from features being available for certain conditions but not for others. Eventually the grid generation and solving process was understood and trustworthy answers were achieved.

After the gridding and solving process was understood, more effort was put into creating an improved geometry for the wind tunnel model. One of the initial problems was the way ICEM discretized curves larger than a certain length. After a certain length was reached, the curve fit created by ICEM was piecewise linear to the point where the piecewise sections were larger than the grid used. As a result, the $\mathrm{Cp}$ values had an unwanted visible wave for each section. The waviness was solved by using multiple smaller independent curves to span the long forebody. The next modification was performed after inspecting the wind tunnel model and noticing the lip on the aft section.

This was discussed earlier in section 5.1.3. Finally the different wind tunnel and the 
freestream boundaries were modeled. The whole process was iterative and each time something new was discovered, the geometry was modified and the flow solver was run again.

\subsection{CFD Run Matrix}

The CFD test matrix, comprised of 10 cases, was not as comprehensive as was the wind tunnel model test matrix which had 54 different runs. The goal was to see if the CFD could accurately match the wind tunnel data and if the trends are in the same direction. The plan was to start with a base configuration and vary one parameter to see how it affected the results. We started with a base geometry that has cusp A and middle slot gap of $0.385 "$. This was done for the free stream grid and then repeated for the straight tunnel and the tunnel with the contraction. From these results we can tell the difference the tunnel contraction has on the results and then move forward with the condition that gives the best data that fits with Roepke and Thomason's wind tunnel results. After finding the best farfield geometry, that geometry was used for the rest of the tests. The next runs were aimed at finding the minimum fan pressure needed for flow attachment at the baseline configuration. Afterwards, results for four different turbulence models were compared. Finally a grid independence study was done to verify that the results were sufficiently independent of the domain discretization. This CFD test matrix can be seen in Table 4 below. 
Table 4: CFD test matrix

\begin{tabular}{|c|c|c|c|c|c|}
\hline$\#$ & Mesh & Gap (in) & Cusp & Turbulence Model & Fan Speed ( $\Delta \mathrm{P}[\mathrm{pa}])$ \\
\hline 1 & Freestream & 0.385 & $A$ & $k-\omega S S T$ & 0 and 500 \\
\hline 2 & Straight Tunnel & 0.385 & $A$ & $k-\omega$ SST & " " \\
\hline 3 & Contraction Tunnel & 0.385 & A & $k-\omega$ SST & “ \\
\hline 4 & Straight Tunnel & 0.385 & A & $k-\omega$ SST & Found for attachment \\
\hline 5 & “ " & 0.385 & A & Sparlart-Allmaras & 0,300, and 500 \\
\hline 6 & $"$ & 0.385 & $A$ & k- $\varepsilon$ realizable & " " \\
\hline 7 & $"$ & 0.385 & A & Reynolds Stress & $"$ \\
\hline 8 & Straight Coarse (67k) & 0.385 & A & $k-\omega$ SST & 0 and 500 \\
\hline 9 & Straight Mid (126k) & 0.385 & $\mathrm{~A}$ & $k-\omega S S T$ & “ " \\
\hline 10 & Straight Fine (230k) & 0.385 & $A$ & $k-\omega$ SST & " " \\
\hline
\end{tabular}

All cases were run at standard sea level conditions with $21.2 \mathrm{~m} / \mathrm{s}$ free stream velocity. This was done because the $30 \mathrm{~m} / \mathrm{s}$ data showed that the flow never was fully attached to the aftbody for any of the fan speeds.

\subsection{Goldschmied Body 20 m/s CFD Results}

First we looked at the freestream condition solution to get an idea on how the body behaves and digress into changing different parameters to see how the propulsor behaves. This is done mainly by looking at the $\mathrm{Cp}$ distribution and fan speeds while changing the tunnel shape and turbulence models to find their effects on the total drag on the body.

\subsubsection{Initial Freestream Solution}

The first solution that ran was the freestream grid with the intermediate slot gap of 0.385 ", cusp A, k-w SST turbulence model at both fan off and 500 pa pressure rise across the fan. This fan pressure rise was high enough that the flow reattached to the aft section, allowing us to compare the separated fan off case to the attached fan on case. 
In the first image below, Figure 55, we can see filled contours for the two initial cases with velocity magnitudes ranging from 0 to almost $30 \mathrm{~m} / \mathrm{s}$. The front of the body had a low speed region leading up to the stagnation point at the nose. The flow then accelerated over the top of the body. For the fan off case, the flow separated in front of the slot entrance. For the fan on case, we see that the flow stayed attached, but still dramatically slowed down over the aft body. The flow that was brought into the suction slot sped up as it leaves the body. At the exit of the jet we see the highest velocity, around $28.8 \mathrm{~m} / \mathrm{s}$, which is about $35 \%$ above freestream velocity.

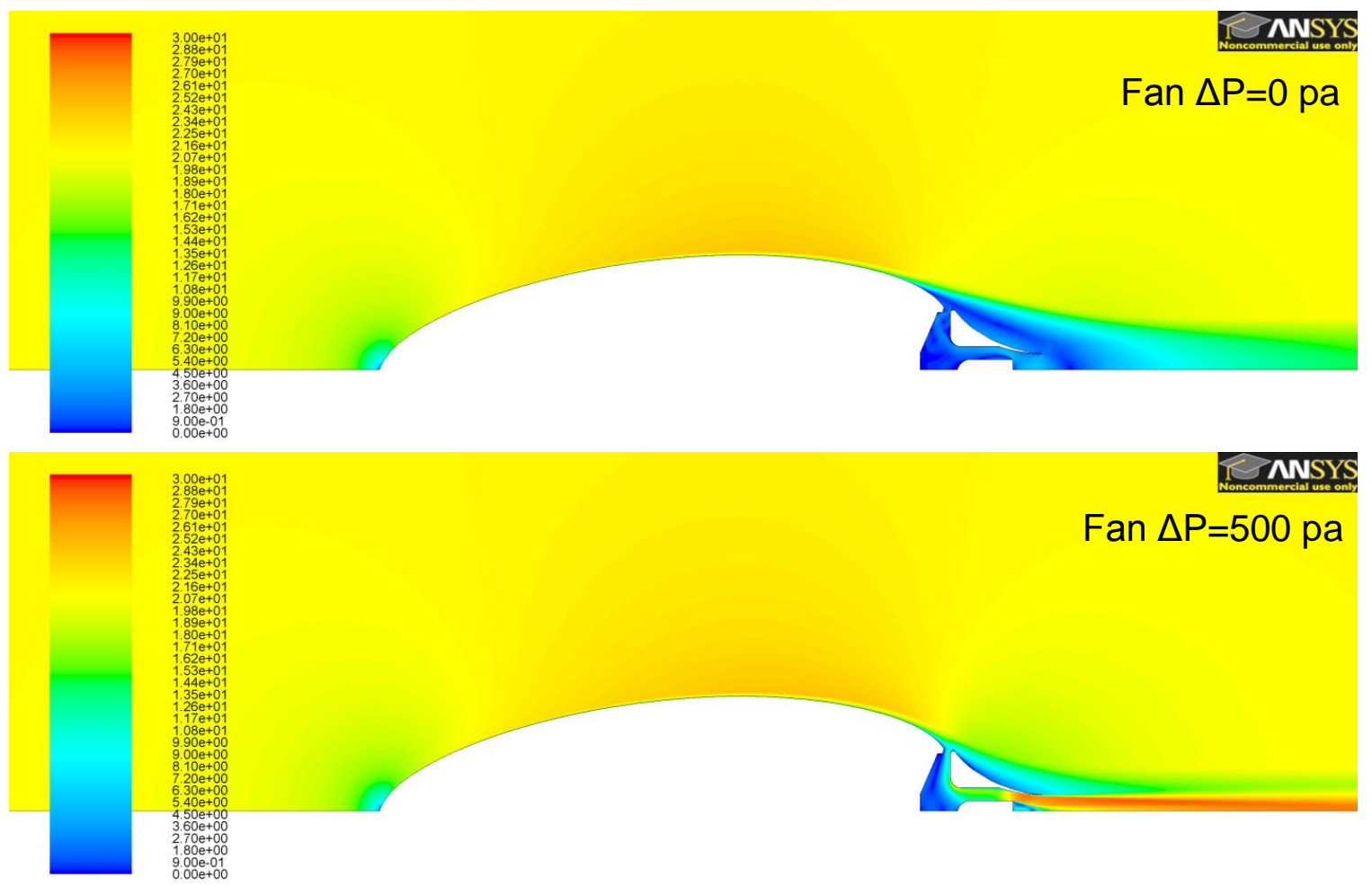

Figure 55: Velocity contours $(\mathrm{m} / \mathrm{s})$ for fan off and fan on at 500 pascals

The next image, Figure 56, shows the pressure distribution for these flows. Both flows have a high static pressure at the nose, due to the front stagnation point, then low 
pressure at the thickest section as the flow accelerated around the body. For the fan off case, there is little pressure recovery before the flow separated. In the separated region, the pressure is relatively constant. This pressure differential, from front to rear, accounts for a large portion of drag and was what the Goldschmied body aimed to reduce. For the fan on case, we can see a large pressure jump in the internal sections. Also, there was a lot higher pressure on the aft section, due to the re-attached flow. This is exactly what Goldschmied was aiming for. There is one important item to note: as the fan is turned up to higher speeds, low pressure region on the aft section of the body (seen in dark blue) moves aft of the area of maximum thickness and closer to the slot entrance. This is very important, as the low pressure on the aft section increased the pressure drag for that section. This will be discussed in more detail later.

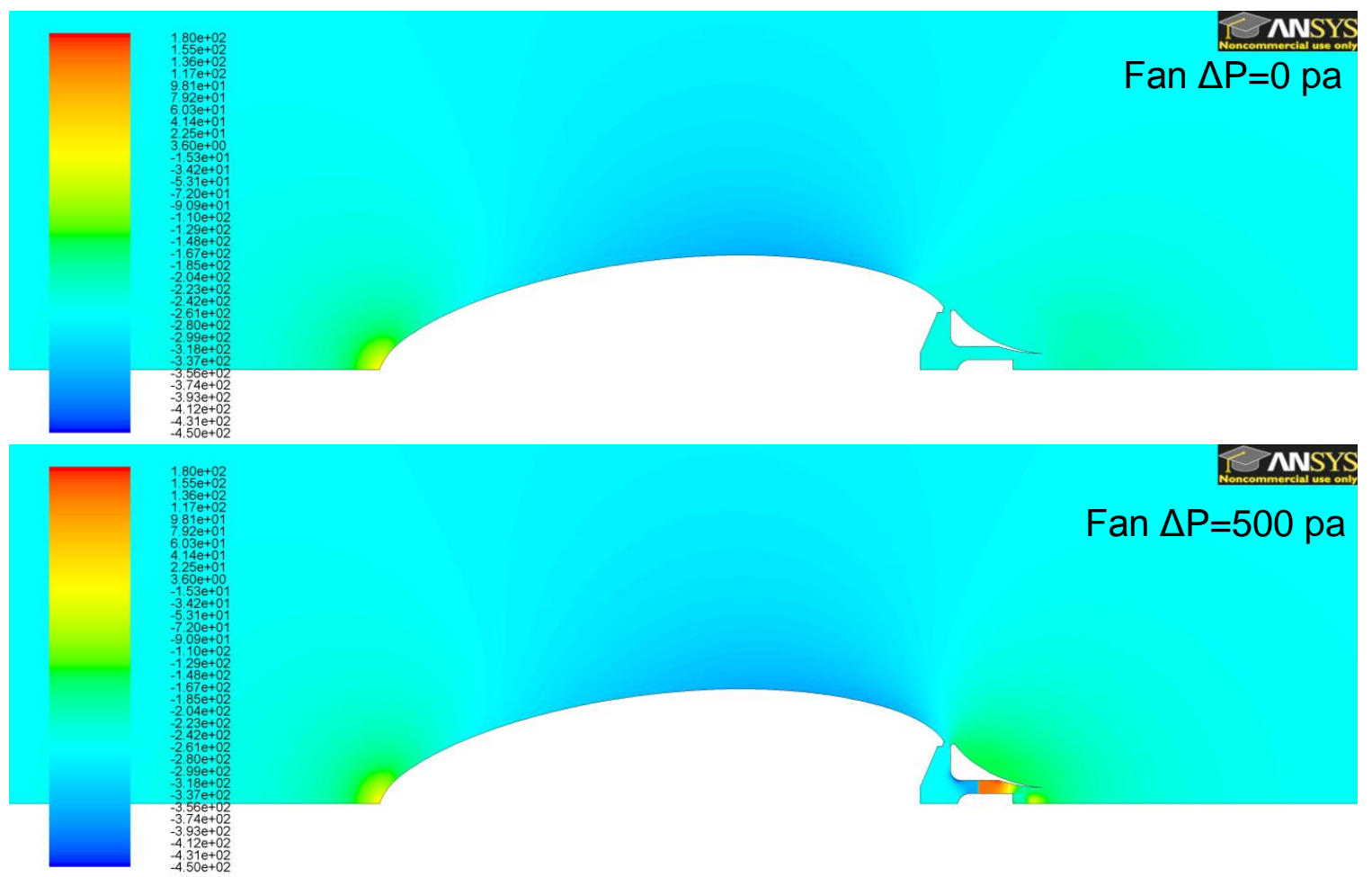

Figure 56: Pressure contours (pascals) for fan off and fan on at 500 pascals 
The next image, Figure 57, looks at the streamlines going around the body. The streamlines are colored by velocity to show fast and slow moving sections. For the fan off case we saw that the circulation in the separated region went back through the slot in the opposite direction. This allowed for the flow to come around the trailing edge, enter the slot, and exit through the slot entrance. This is consistent with the experimental data, as the slot was left open and allowed for the backflow. For the fan on case, the slot sucked in the boundary layer streamlines and brought them through the slot. The faster moving flow is then brought to the aft body and is slowed down from the diffuser effect of the aft section.

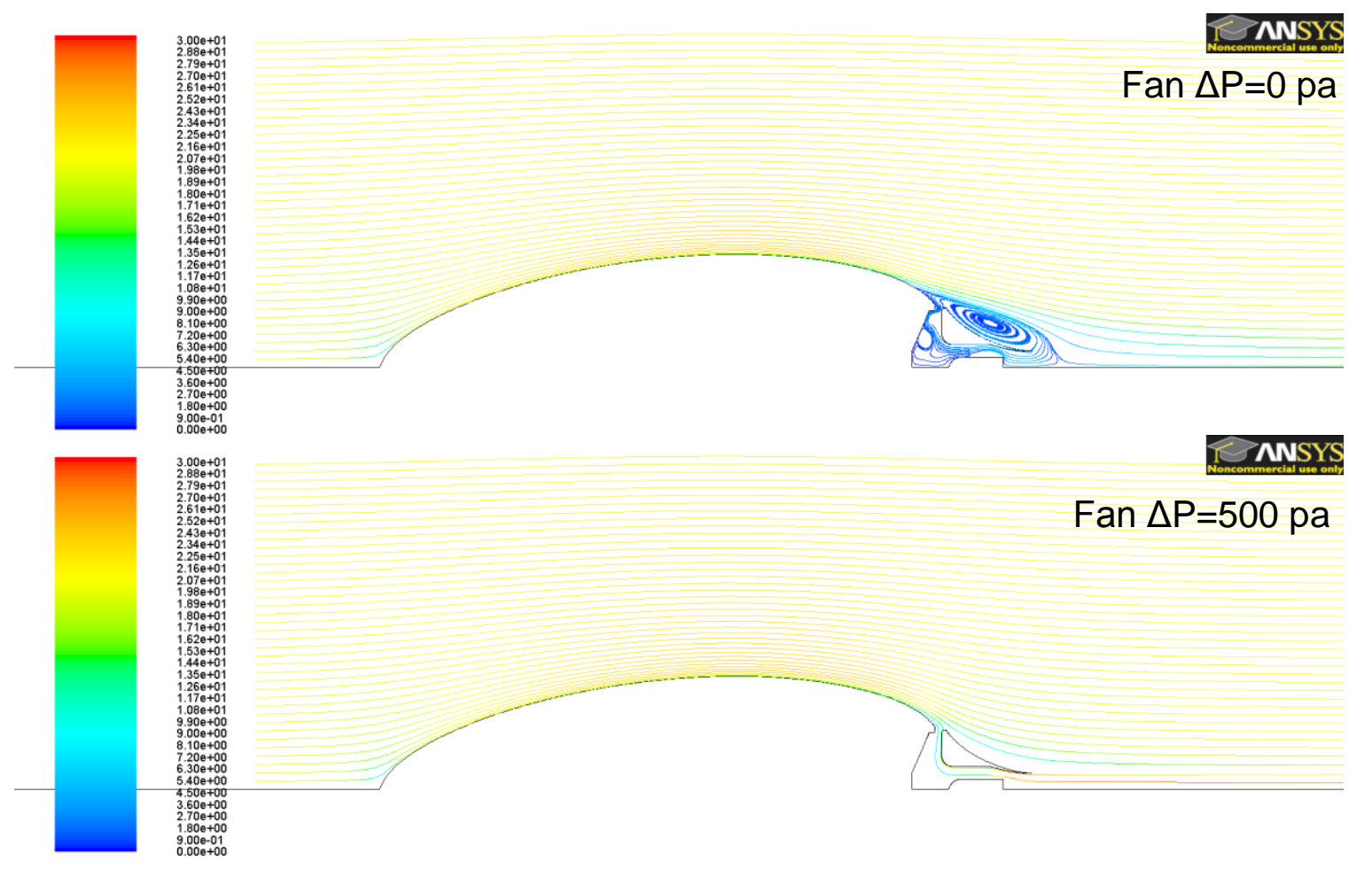

Figure 57: Streamlines colored by velocity $(\mathrm{m} / \mathrm{s})$ for fan off and fan on at 500 pascals

Figure 58 is a close-up of the aft streamlines, giving more details on the aft region and the flow through the internal slot. 


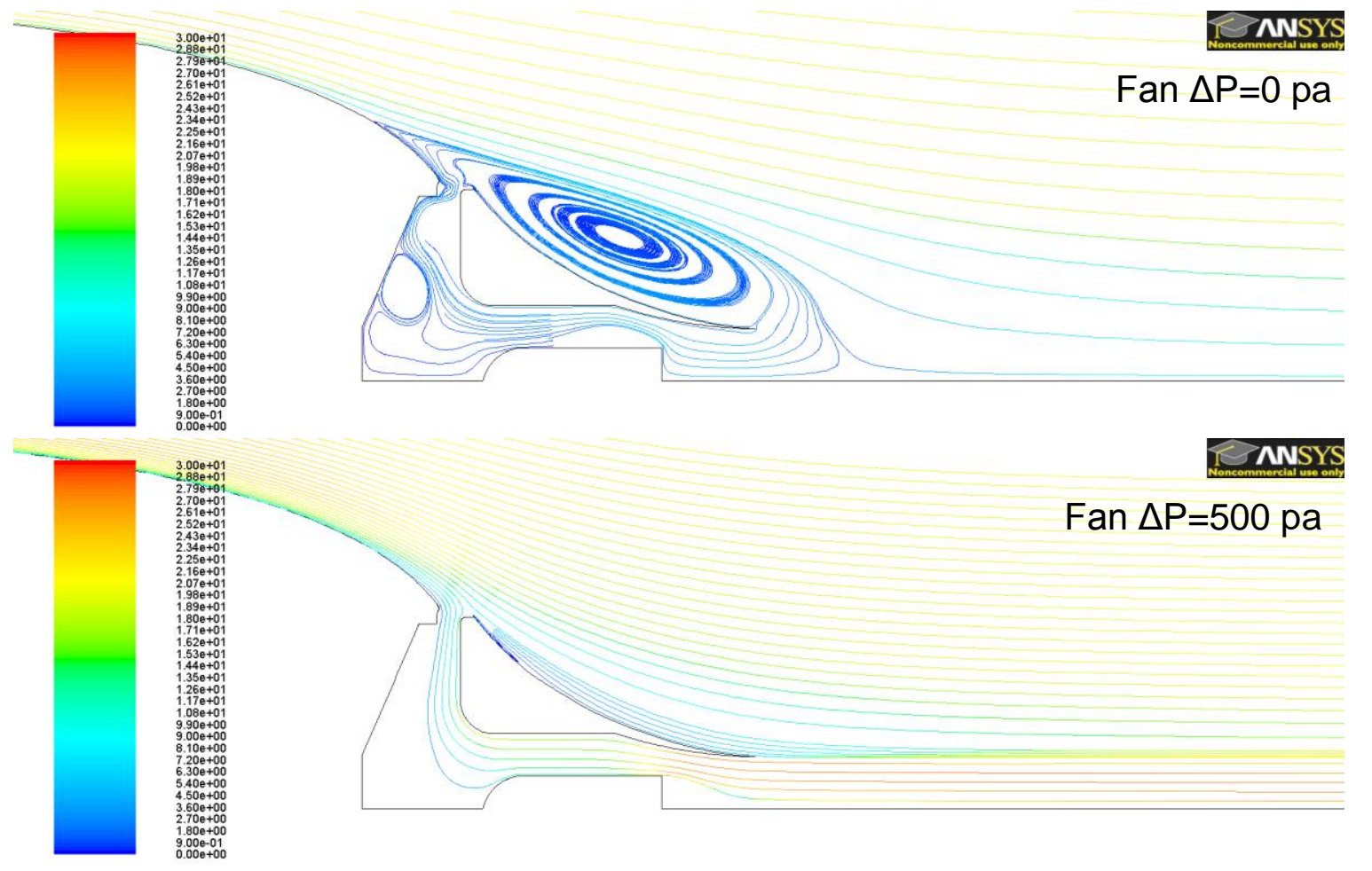

Figure 58: Close up rear streamlines colored by velocity $(\mathrm{m} / \mathrm{s})$ for fan off and fan on at 500 pascals

From the figure above we see that there is a small separated region right after the slot for the fan on case. A close up of this can be seen in Figure 59 below. As the flow is brought into the suction slot there is a turning of the flow, and because of the flat, blunt geometry, there is a stagnation point. The flow then has to turn past this flat section and either enter the suction slot or move to the aft section. When moving aft, the flow has to move over the small lip on the backside of the entrance. The lip caused the flow that is moving over the aft section to form a small separation bubble. The flow then reattached after this separation bubble. The reattachment can be seen in the previous streamlines in Figure 58 above. 

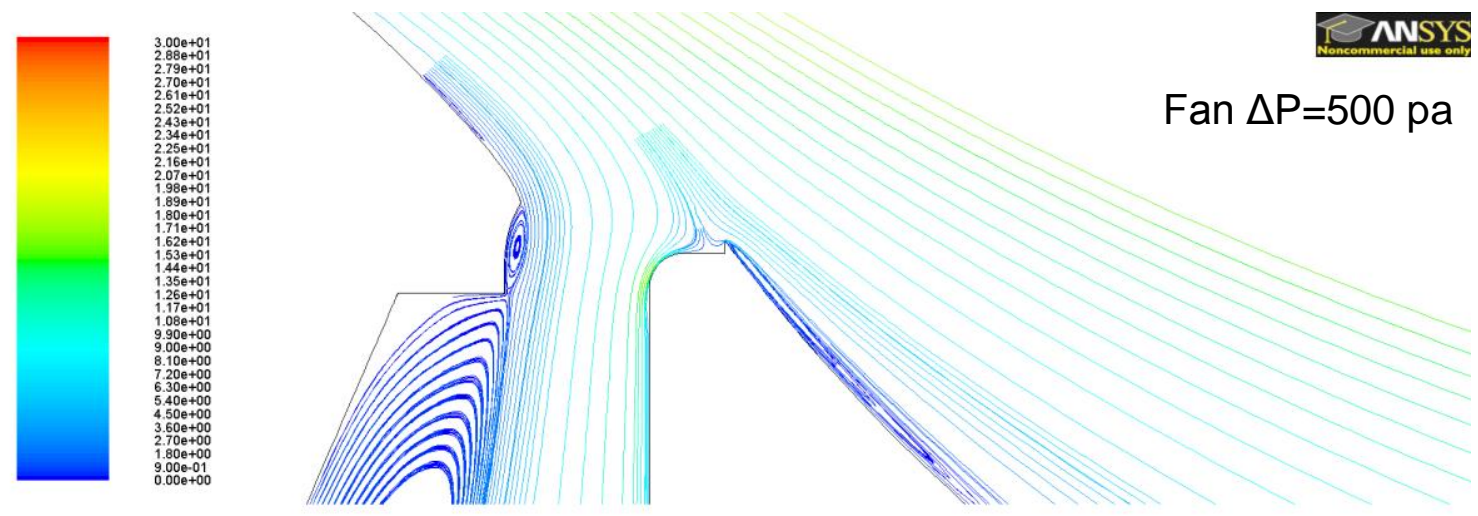

Figure 59: Inlet streamlines colored by velocity $(\mathrm{m} / \mathrm{s})$ for fan on at 500 pascals

Another interesting item was that the cusp was holding a trapped vortex, as was described by Goldschmied and Ringleb. There was not enough time to test different cusp or no cusp designs to find the importance of it, but it does seem to help bring the flow around the corner and into the slot.

\subsubsection{Tunnel Effects}

The next item tested was the effects of the wind tunnel boundary walls on the coefficient of pressure and the overall drag of the body. Figure 60 below shows the velocity contours for the straight tunnel geometry. The slow moving boundary layer on the tunnel walls can be seen in greenish/blue the top section of the figure. Besides this change, the rest of the flow looked relatively similar to free stream conditions as covered in the previous section. 


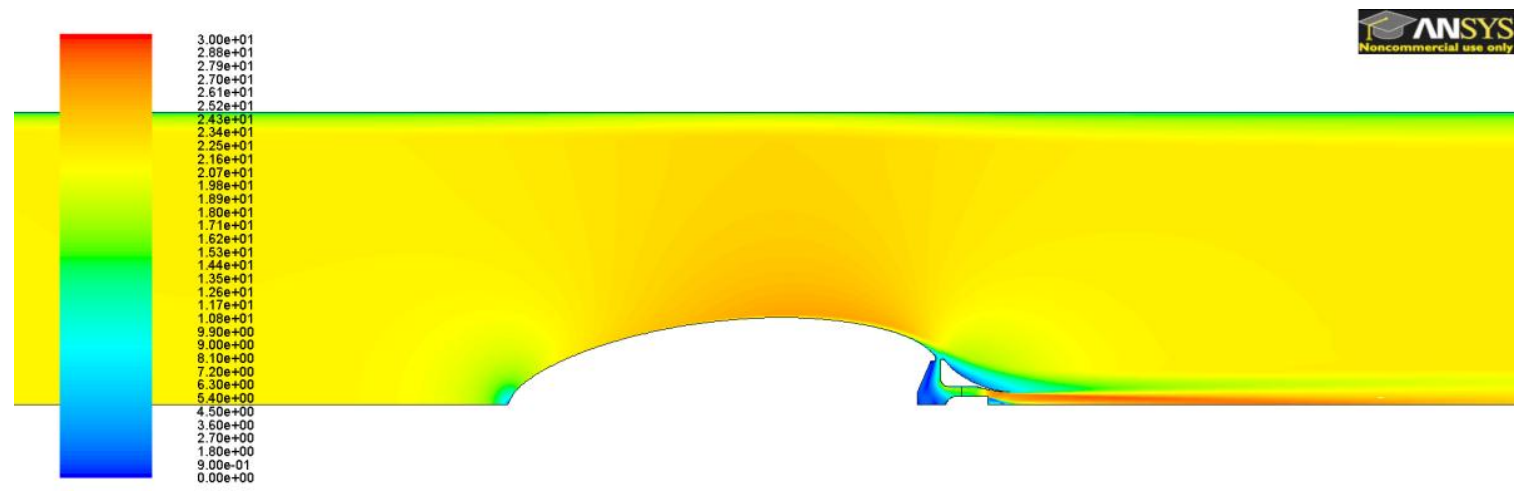

Figure 60: Velocity contours $(\mathrm{m} / \mathrm{s})$ for straight tunnel geometry

The velocity profile can also be seen for the tunnel geometry with the tunnel contraction modeled in Figure 61 below. This geometry was examined to see if the contraction led to uneven velocity distributions and radial flow components at the nose of the model.
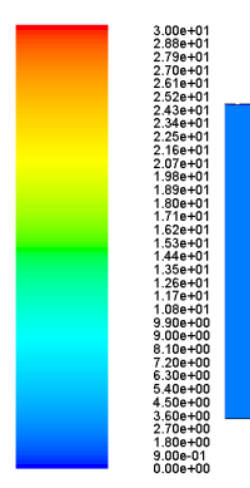

INNSYS

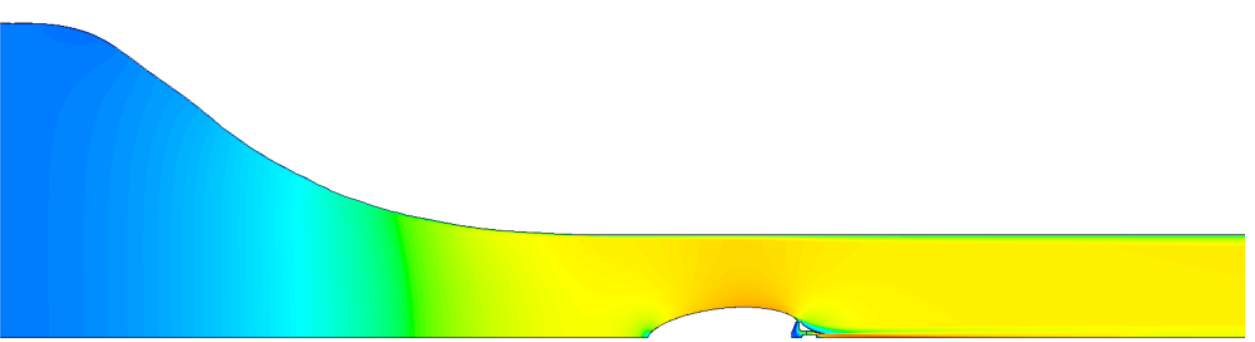

Figure 61: Velocity contours $(\mathrm{m} / \mathrm{s})$ for tunnel contraction geometry

The effect of these different tunnel geometries can be seen in the pressure distributions on the body in Figure 62 and Figure 63. The $\mathrm{Cp}$ starts off at the stagnation point, but does not drop as low through the area of maximum thickness as observed for the wind tunnel models. At the lowest pressure, the freestream $\mathrm{Cp}$ is about 0.1 higher than the two wind tunnel cases. 
We see that the two tunnel geometries have nearly the same $\mathrm{Cp}$ curve for both the power on and power off settings. This showed that the contraction has very little effect on the pressure distribution.

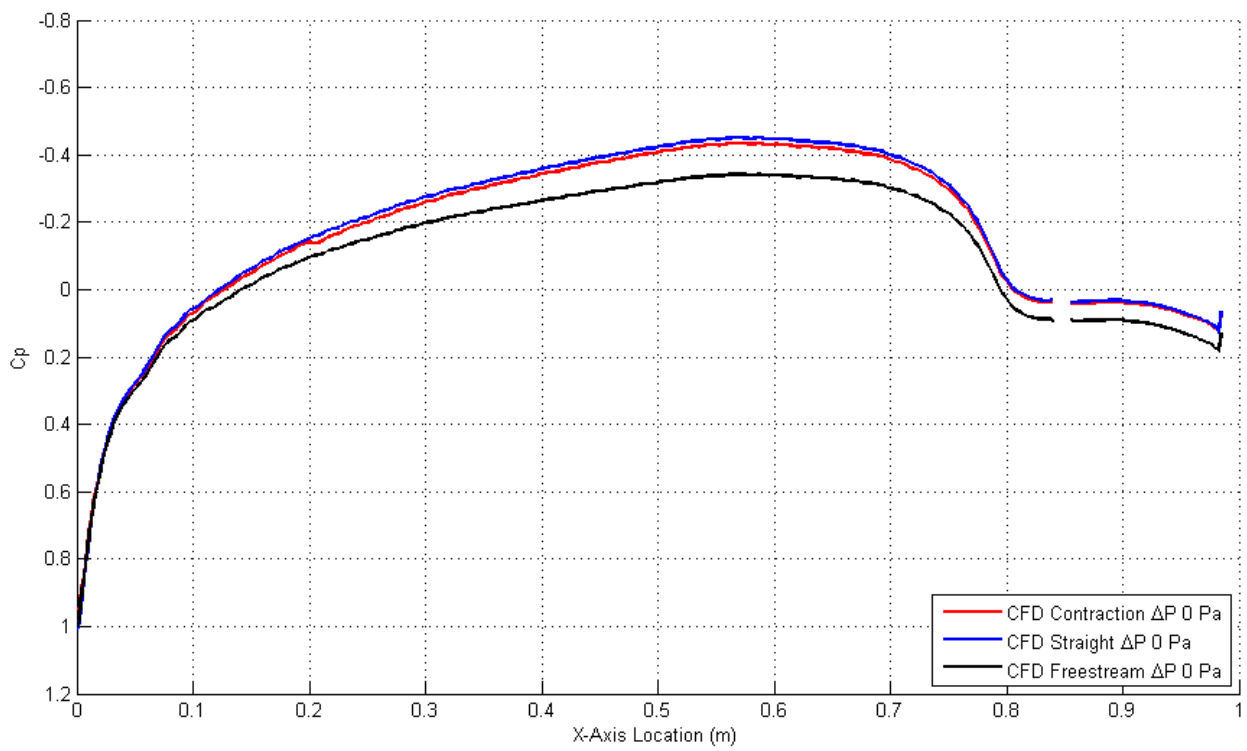

Figure 62: Tunnel effects on body $\mathrm{Cp}$ for fan off conditions

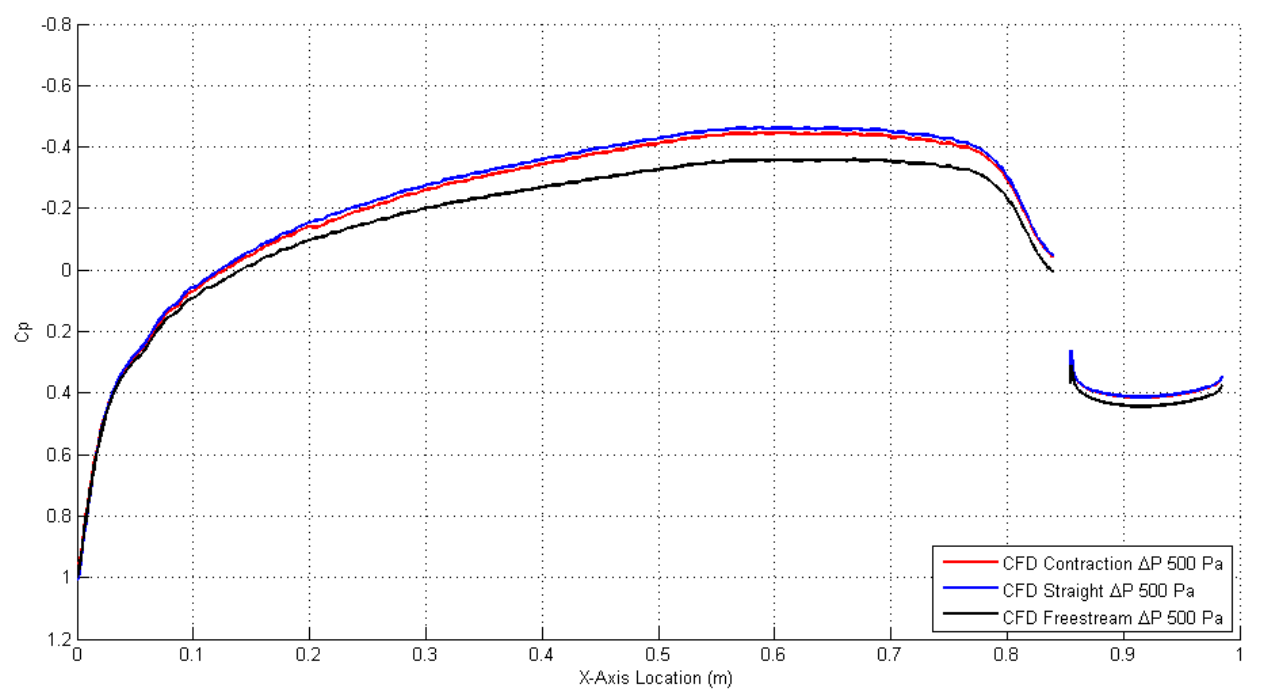

Figure 63: Tunnel effects on body $\mathrm{Cp}$ for fan on at 500 pascals 
The differences in the pressure resulted in different drag values for each case. The fan off freestream had a total drag of 0.0397 and the straight tunnel and contraction had drag values of 0.0524 and 0.0526 . Similarly the fan-on condition had the same effect: the two tunnel conditions were nearly the same in drag and the freestream was about 110 counts less. The differences in drag for the three different far-field boundary conditions are summarized in Table 5 below.

Table 5: Comparison of drag coefficients from free steam to wind tunnel conditions for both fan off and fan on at 500 pascals

\begin{tabular}{|c|c|c|c|c|c|c|}
\cline { 2 - 7 } \multicolumn{1}{c|}{} & \multicolumn{3}{c|}{ Fan Off } & \multicolumn{3}{c|}{ Fan On: 500 Pascals } \\
\cline { 2 - 7 } \multicolumn{1}{c|}{} & Free & Straight & Contraction & Free & Straight & Contraction \\
\hline Pressure Drag & 0.0174 & 0.0275 & 0.0294 & 0.0185 & 0.0268 & 0.0284 \\
\hline Skin Friction & 0.0223 & 0.0250 & 0.0232 & 0.0249 & 0.0277 & 0.0258 \\
\hline Body Total & 0.0397 & 0.0524 & 0.0526 & 0.0434 & 0.0545 & 0.0542 \\
\hline Fan & 0.0000 & 0.0000 & 0.0000 & -0.0385 & -0.0385 & -0.0385 \\
\hline Total & 0.0397 & 0.0524 & 0.0526 & 0.0048 & 0.0160 & 0.0157 \\
\hline
\end{tabular}

The higher drag for the wind tunnel models was mainly from the pressure drag. This was because the additional buoyancy effects in the wind tunnel. The buoyancy effect is a result from a decrease in pressure as the flow is accelerated by the constriction from the growing wall boundary layer. This results in a lower pressure on the rear of the model than the front. The buoyancy named come from the observation that it behaves similarly to the effect of an immersed body in a gravitational field (like water), the difference is that the force acts in the direction of the flow, not the direction of gravity. The effects on the pressure in the tunnel can be seen in Figure 64, where the tunnel wall Cp vs. location can be seen. The low pressure region around $-0.5<x<1.5$ was due to the flow around the model. The constant decrease in $\mathrm{Cp}$ was a result the growing boundary layer on the tunnel walls. 


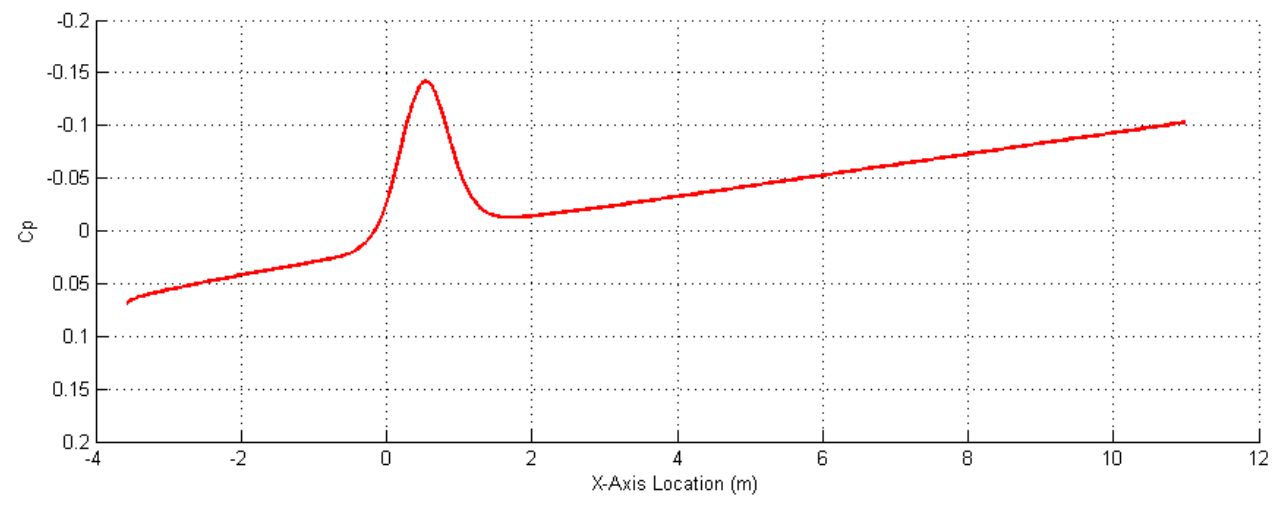

Figure 64: Straight tunnel wall $\mathrm{Cp}$

The two cases with wind tunnel walls also gave a higher skin friction drag then the free air case. This was due to the fact that with the addition of the wall: the boundary layer on the body was not able to expand as much, resulting in thinner boundary layer, and the flow between the wall and the body was accelerated more than in freestream conditions. These two factors led to higher local skin friction at the wall, which in turn led to higher overall skin friction drag.

From the table we saw that the tunnel walls did have a large effect on the results as compared to the free air case, but that the tunnel contraction was not much different than the straight tunnel. Because of the differences between freestream and the tunnel, with that of the collected wind tunnel data, the straight tunnel geometry will be used for the remainder of this report.

\subsubsection{Fan Effects}

The next item looked at was the effect of the fan pressure rise on the aft body attachment, pressure distribution, and drag for the body. The fan pressure increase was varied from 0 to 1500 pascals in $100 \mathrm{~Pa}$ increments from $0 \mathrm{~Pa}$ to $600 \mathrm{~Pa}$, and $1000 \mathrm{~Pa}$ and $1500 \mathrm{~Pa}$ for the higher values. 
Figure 65 shows the effects of the fan by plotting the Cp of the outer fore and aft surfaces for the nine different fan settings. Looking at the aft section we saw that the initial fan settings produce a large change in the pressure recovery, up to about $300 \mathrm{~Pa}$. After this point, it seemed that the flow was mostly re-attached and the increases in pressure recovery after this point were small compared to the previous jumps. This increase in pressure recovery was what Goldschmied was looking for, as high pressure on the aft section gives the desired decrease in pressure drag of a body. Another observation was that the suction flow created by the fan dramatically changes the fore body pressure leading up to the slot, with the changes being seen as early as $50 \%$ chord, $35 \%$ chord length in front of the slot. We saw that as the fan pressure rise was increased, the Cp stayed low, around -0.45 , for a longer time. This brings the adverse pressure gradient further back, which delyaled the onset of separation, but also created low pressure on the aft side of the maximum radius. This decrease in pressure on the aft side of the maximum radius caused the pressure drag in that section to rise, which will be discussed later. 


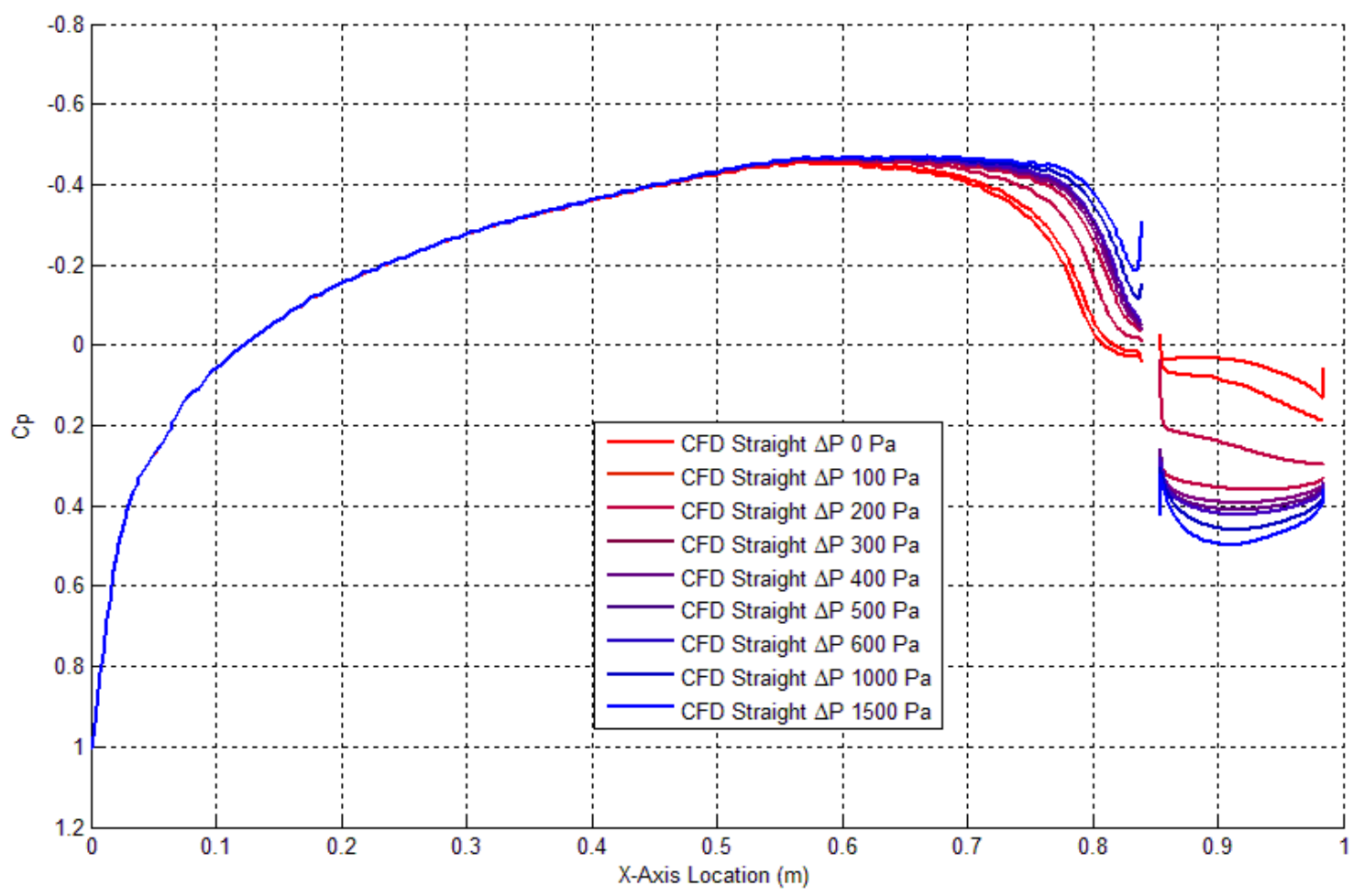

Figure 65: Cp disribution for fan pressures of 0 to 1500 Pascals

One way to visually look at how the pressure changes effect the drag of the vehicle is through a $R^{2}$ vs. Cp plot. The $R^{2}$ value is used because the body is axisymmetric and that as you move out radially the area of each 'station' is proportional to $R^{2}$. The fore and aft sections of the body can be then plotted on the same plot. At a single radius, if the front pressure is higher than the aft pressure there is a drag force associated with that pressure differential. If it is the other way around, with a higher pressure on the aft section, there is a thrusting force associated with it. The areas associated with each region are related to the total body pressure force. $R^{2}$ vs. Cp plots can be seen for each of the nine fan attachment cases in Figure 66. 

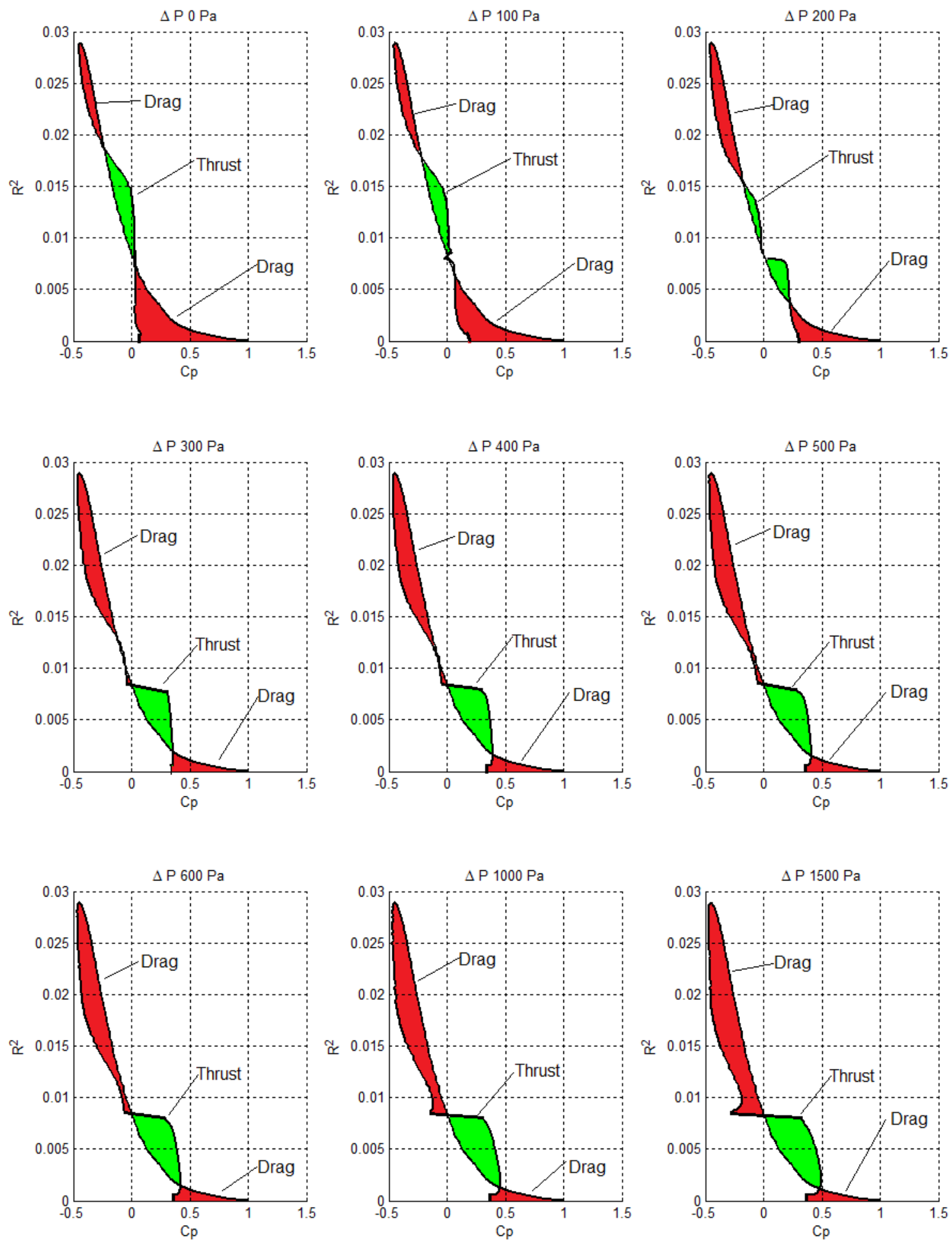

Figure 66: Cp vs. $R^{2}$ values for varying fan pressures 
From Figure 66 we see that an increase in fan speed does decrease the drag and increase the thrust along the body aft of the suction slot (below approximately $\left.R^{2} \sim 0.08\right)$. This increase comes at a huge cost in increased drag in front of the suction slot. The pressure on the area aft of the maximum thickness, but in front of the suction slot is decreased enough, that at higher fan pressures, it changes the once drag/thrust region to a drag-dominated region that increases as fan pressure differential increases.

To better explore the effects of changes in fan pressure rise, each component of drag was broken up and plotted independently. The first plot, Figure 67, shows the break-up of the forces contributing to the drag seen on the CFD model. It breaks the pressure forces up into the forebody (all the area in front of the slot), the aft body (all the area behind the slot) and the slot (all of the internal slot areas) as seen in Figure 69. The next force is the skin friction for the whole body. All of these are added together to get the 'Total Body Forces'. The first thing we see in the plot is that the axial force on the forebody is increased significantly as fan pressure increases, which relates to a great increase in drag. Next, the aftbody axial force is decreased as the fan pressure is increased. The rest of the forces are relatively constant for the varying fan pressures. When all the forces are added we see that the forebody and aftbody changes nearly cancel each other out, creating a total body force that does not vary as much as the single components. This leads to a nearly constant body drag force that actually slightly increases as fan pressure differential increases beyond $350 \mathrm{~Pa}$. 


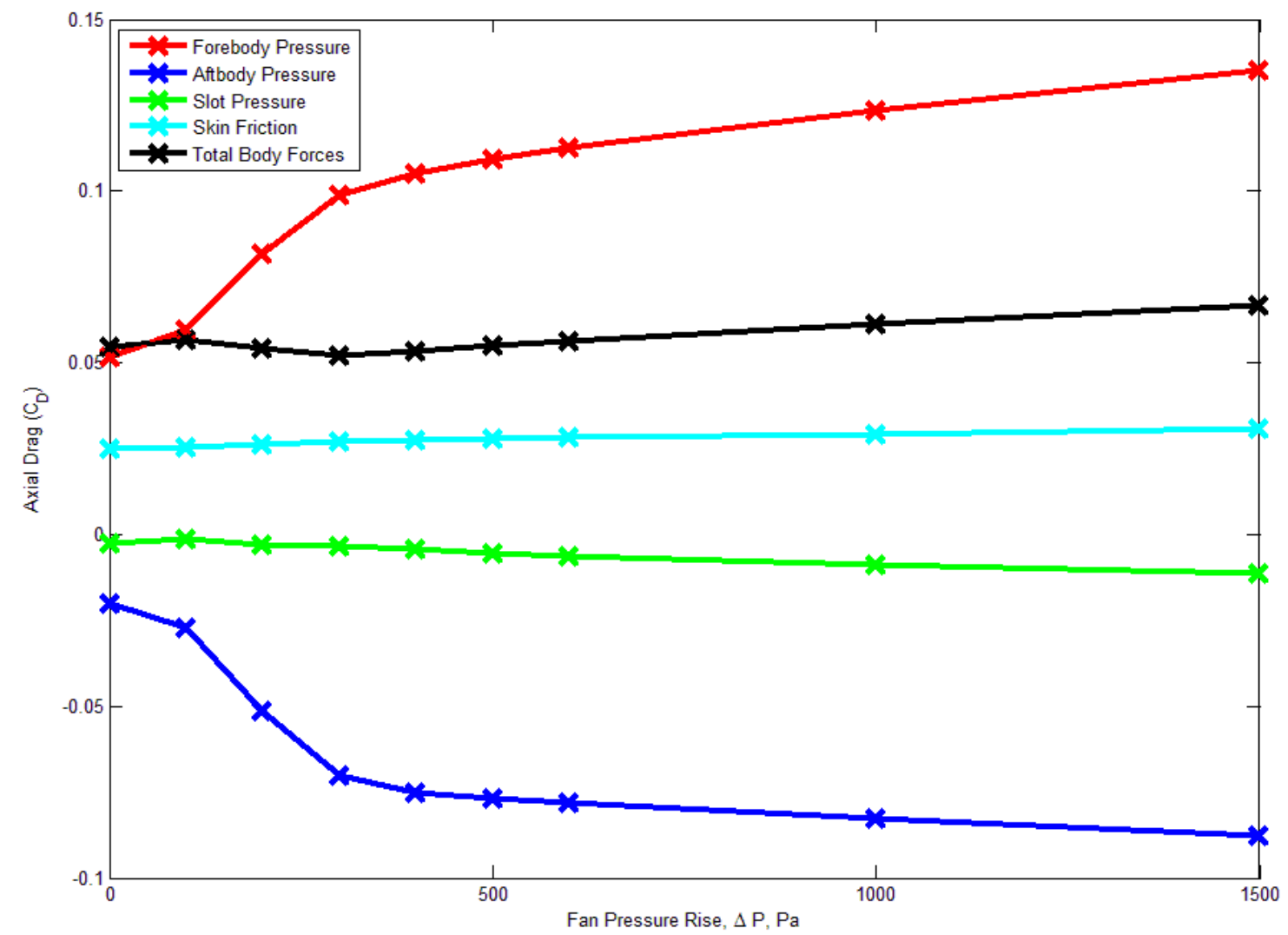

Figure 67: CFD Body axial force build-up for different fan speeds

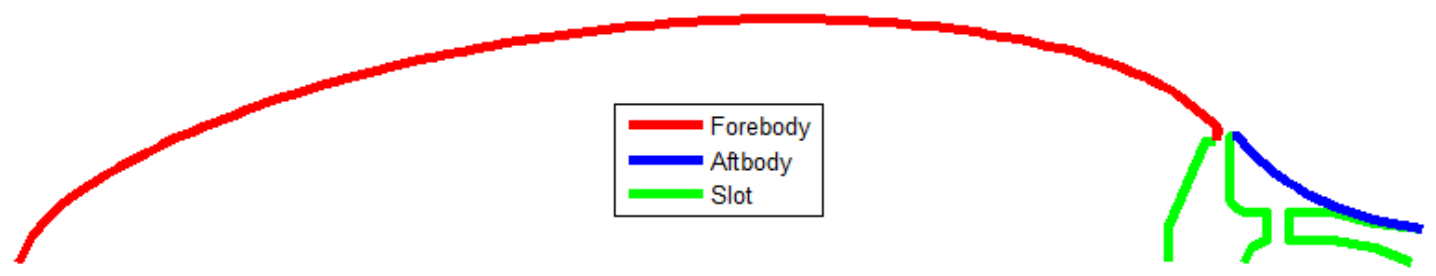

Figure 68: Breakup of body sections between the fore, aft, and slot sectons

The total axial body force can then be added to the force that the fan exerts. The fan force is found by taking the pressure differential across the fan and multiplying by the fan area. Adding the fan and body forces we get the total force on the body, as would be seen by a sting. The plot of this can be seen in Figure 69. As mentioned before, the 
body forces are relatively constant and the fan really accounts for the reduction in axial force, with a zero force estimated at a fan pressure around 750 pascals.

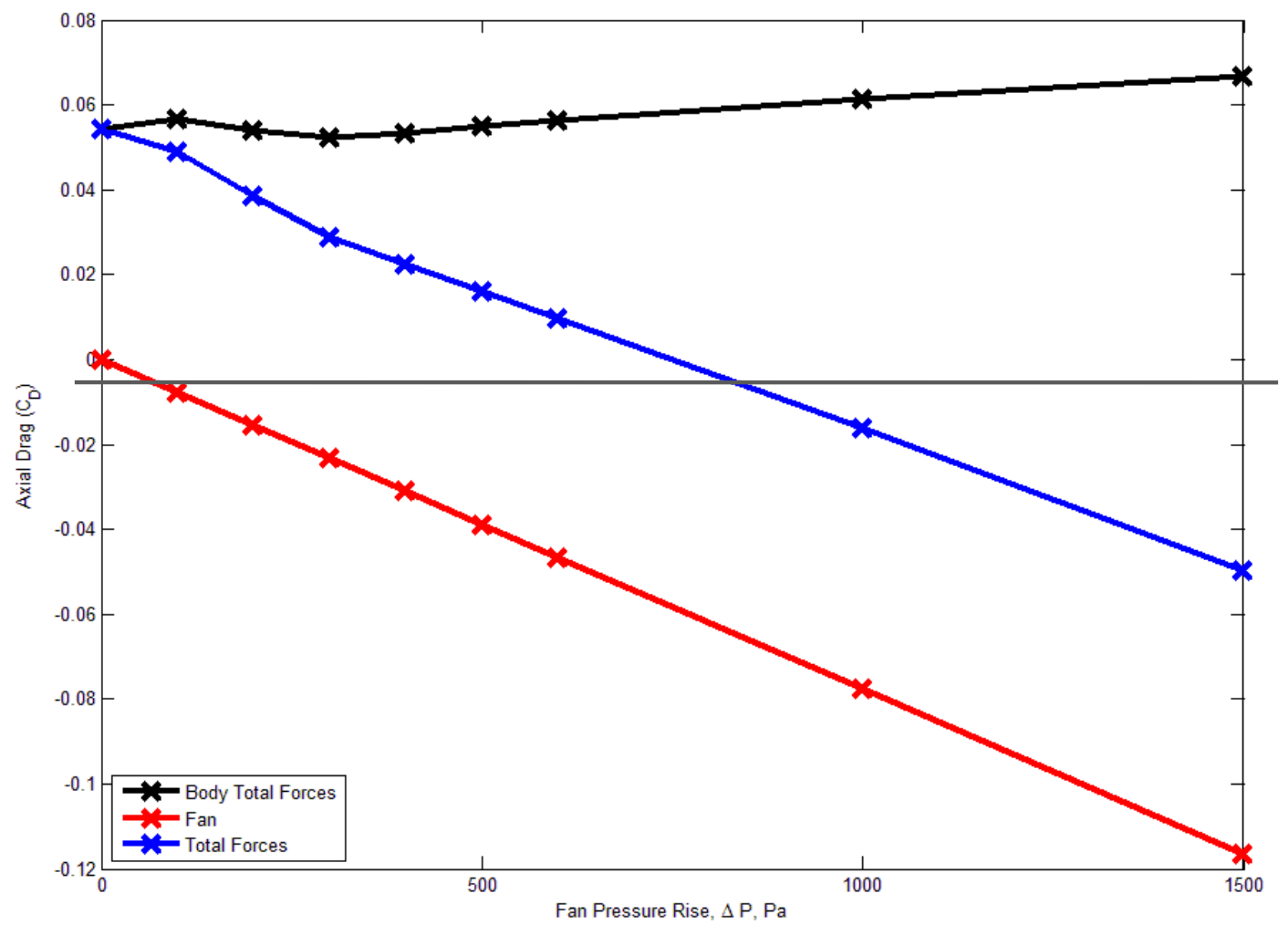

Figure 69: CFD Total force build-up for different fan speeds

Next, the inlet flow was looked at to see how the fan affected that area. Velocity vector plots were taken at every pressure for the slot inlet and then plotted side by side in Figure 70 . With the fan off the flow separated pretty far upstream of the slot and there is the recirculation region as expected for separated flows. An interesting feature is that the flow has reversed through the slot, as part of the circulation region, and there is a noticeable flow rate out of the inlet slot. At the $100 \mathrm{~Pa}$ fan pressure rise the flow has switched back to the predicted direction, but the flow is not strong enough to change the separated region much. Rather it changes the recirculation path and location. At $200 \mathrm{~Pa}$ we see that the slot starts to ingest more flow, but it is still separated before and after the slot. At this speed the streamlines are starting to bend to the aft section, but not enough 
to reattach. At $300 \mathrm{~Pa}$ we see that the flow is brought down far enough to reattach to the aft section, although it is still separated before the slot. The higher fan speeds continue this pattern, bringing the flow closer to the aft section and reducing the forebody separation. Full forebody attachment is obtained at $600 \mathrm{~Pa}$. Another interesting feature observed was that as the flow is turned into the suction slot, there is a part of the flow that is brought radially into the aft body spacer. Running into this causes a stagnation point and the flow to either side of this has to turn and go around each corner. This is not ideal, and along with the rear lip, causes a separation bubble for a small region on the aftbody. This is easier seen with the streamlines in Figure 59.
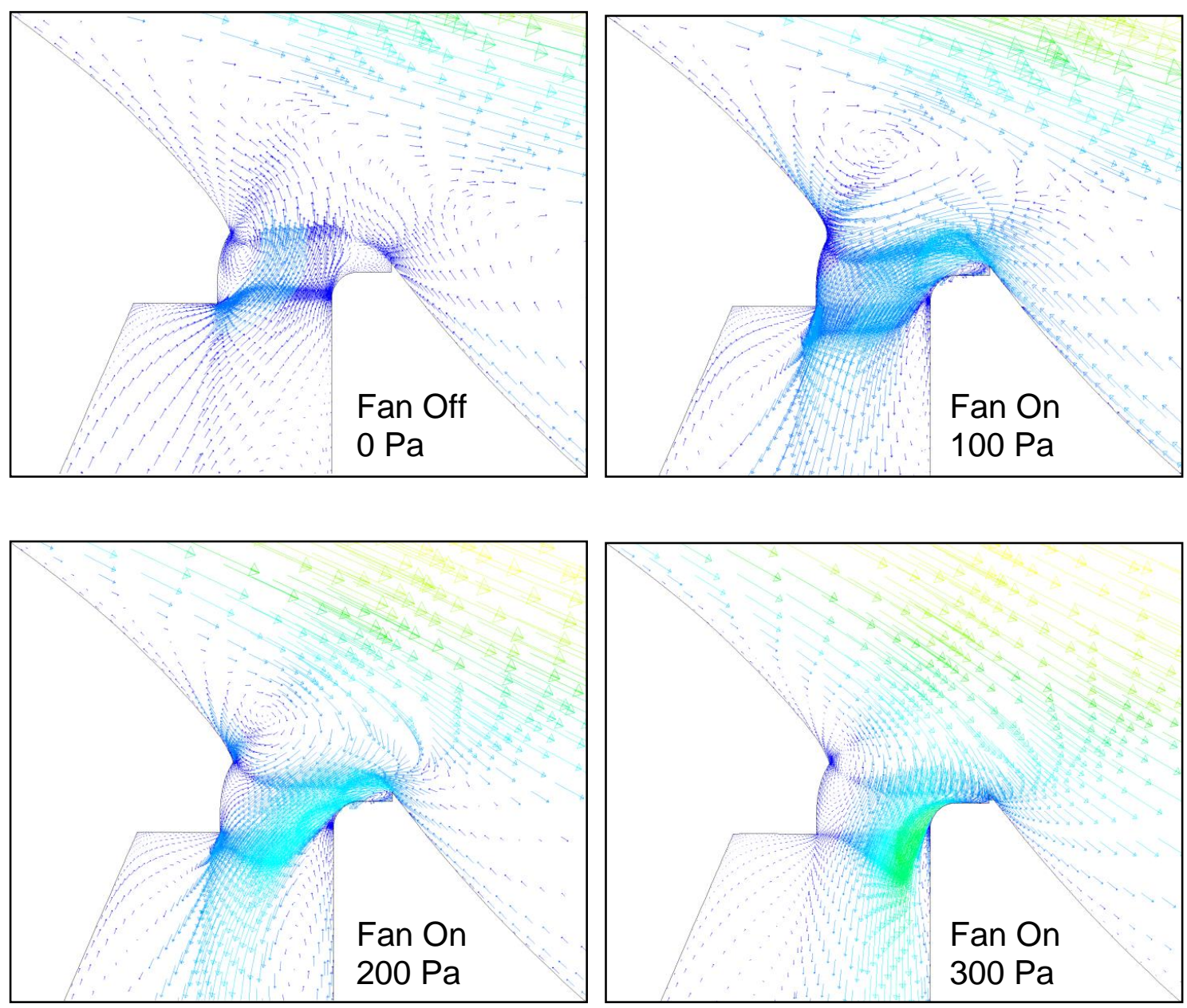

Figure 70: Velocity vector plots showing the flow at the inlet entrance for varying fan speeds 

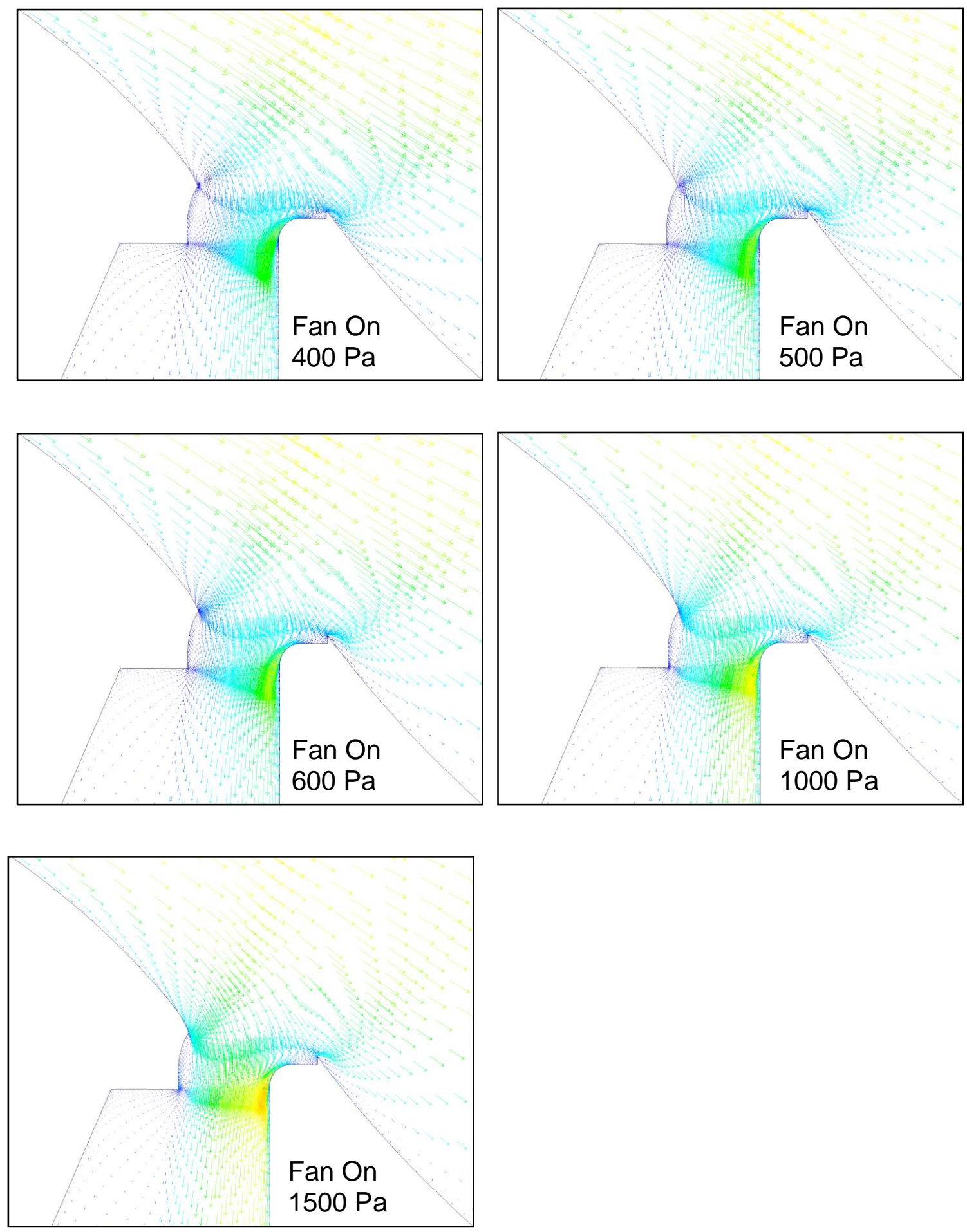

Figure 70: Velocity vector plots showing the flow at the inlet entrance for varying fan speeds 


\subsubsection{Turbulence Model Effects}

Calculations were performed with four turbulence models were looked at to see how this changed the solution. The models looked at were the Sparlart-Allmaras model, the $k-\varepsilon$ realizable model, the $k-\omega$ shear stress transport model, and the Reynolds stress model. More details about each model can be seen in section 7.2, Turbulence Modeling, on page 64 .

Comparing the four models, the Sparlart-Allmaras model (a one equation model) ended up being really far from the other three models, with drag values over double that of the others. This difference can be seen in Figure 71 below.

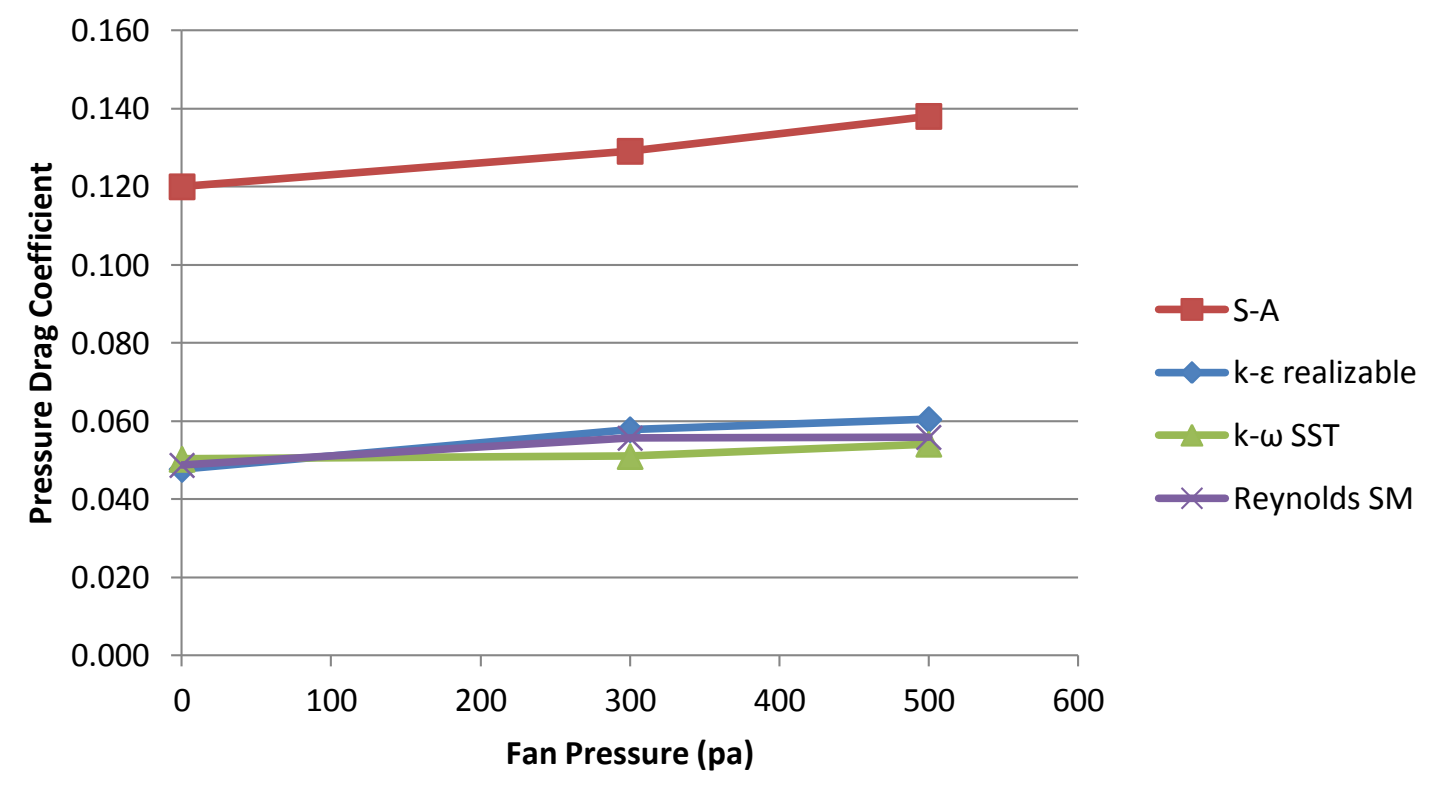

Figure 71: Pressure drag coefficents for different turbulence models at different fan pressures The differences between the $k-\omega$ SST and other turbulence models can be seen below in Table 6 below. 
Table 6: Pressure Drag differences due to turbulence models for the straight tunnel at different fan pressures

\begin{tabular}{|c|c|c|c|c|}
\hline $\begin{array}{c}\text { Fan } \\
\text { Pressure }\end{array}$ & $\begin{array}{c}\mathrm{k}-\omega \mathrm{SST} \\
\left(\mathrm{C}_{\mathrm{d}, \mathrm{p}}\right)\end{array}$ & $\begin{array}{c}\mathrm{S}-\mathrm{A} \\
\text { (\% Diff) }\end{array}$ & $\begin{array}{c}\mathrm{k}-\varepsilon \text { realizable } \\
\text { (\% Diff) }\end{array}$ & $\begin{array}{c}\text { Reynolds SM } \\
\text { (\% Diff) }\end{array}$ \\
\hline 0 & 0.050 & 138.8 & -5.1 & -3.1 \\
\hline 300 & 0.051 & 153.3 & 13.4 & 9.2 \\
\hline 500 & 0.054 & 155.1 & 11.6 & 3.2 \\
\hline
\end{tabular}

The large difference between the Sparlart-Allmaras model and others was concerning, as one would not expect that much difference. The Fluent Theory Guide ${ }^{(27)}$ states that; "The Spalart-Allmaras model was developed for aerodynamic flows. It is not calibrated for general industrial flows, and does produce relatively larger errors for some free shear flows, especially plane and round jet flows." Our model has two areas that the model is not calibrated for: it is more of a general industrial flow, as it is a large pipe and not in freestream conditions. Secondly the exit jet from the fan has a large free shear round jet flow. These un-calibrated flow regimes led to a lower pressure on the rear of the model, resulting in significantly higher drag values.

The closeness of the k- $\omega$ SST model to other models, besides the S-A model, and the previous knowledge that it performs well in separated flows led us to use it for the turbulence model for all the other runs. 


\section{CFD Comparison to Cal Poly's Tunnel Results}

The first value looked at was the comparison of the pressure distributions over the whole body compared to the wind tunnel to see how well CFD was able to predict the flow. The first two plots, Figure 72 and Figure 73, were plotted independently for clarity and then Figure 77 shows data for all power settings for the single cusp and slot width. Even though these plots were at a single geometry, they were representative of the whole data set, and changes from the cusp or slot width were not as pronounced as the variations in pressure distribution caused by fan speed changes.

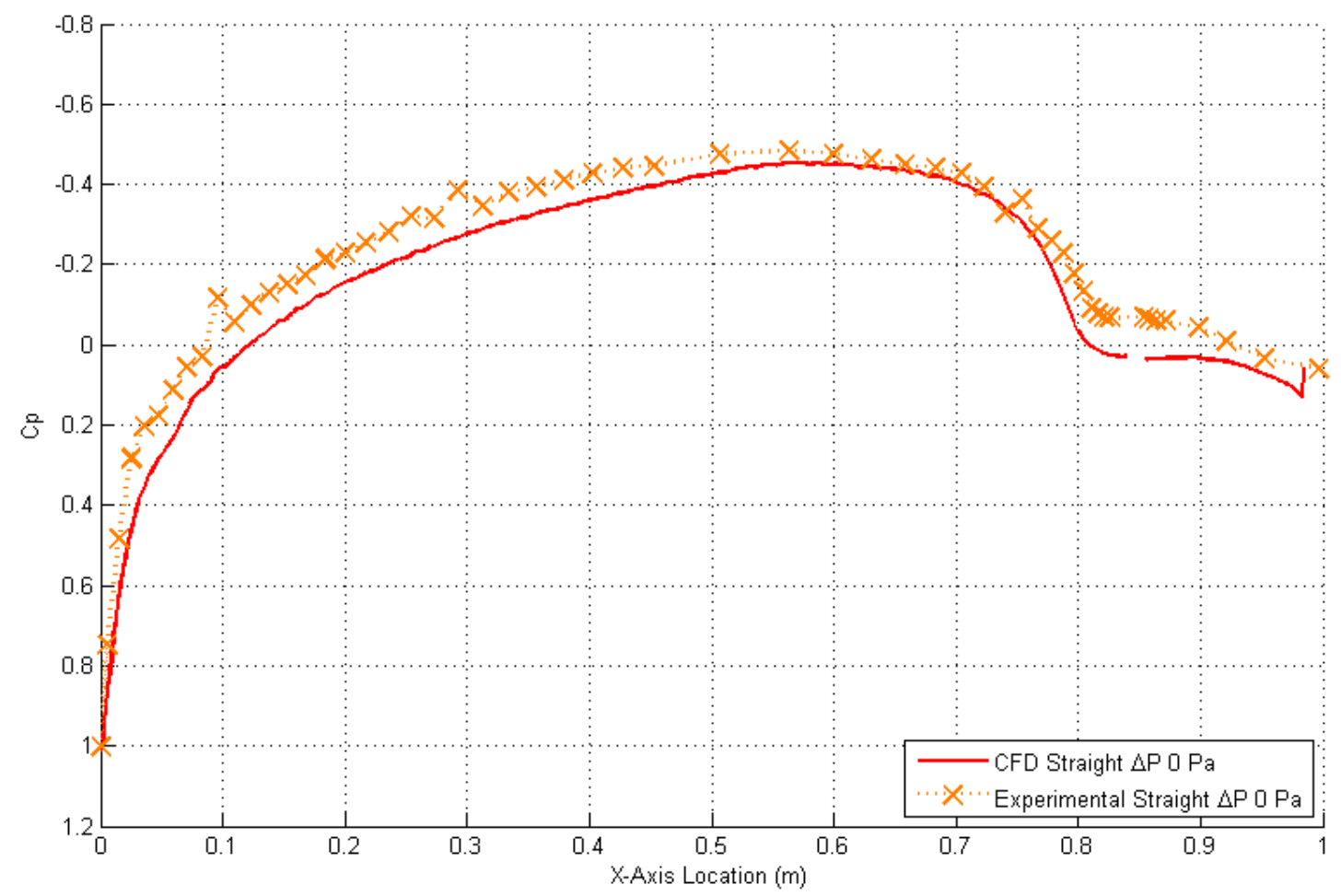

Figure 72: Comparison of Cp CFD data to Cal Poly experimental data for fan off conditions 


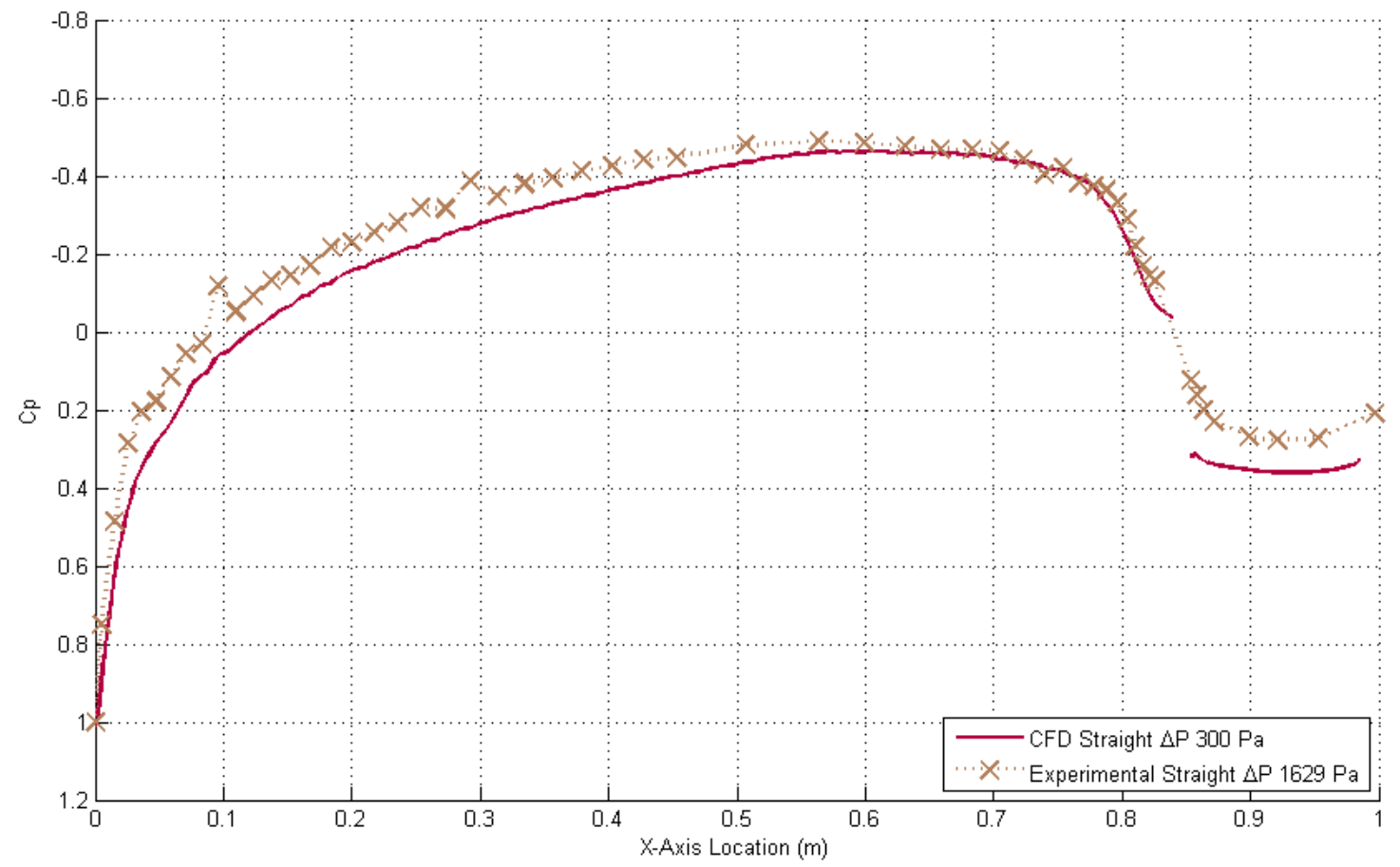

Figure 73: Comparison of Cp CFD data to Cal Poly experimental data for the fan at $300 \mathrm{~Pa}$

The first two plots show pretty good agreement between the CFD and the experiential data, the main difference is the experimental cases had higher $\mathrm{Cp}$ values for almost the entire length. This was interesting for the first half of the body because CFD should have been able to predict the positive pressure gradient accurately over the nose of the body.

The discrepancy may come from several factors; the first is that the static pressure for the model was measured in the tunnel is not constant. This has an effect on the nondimensionalization process different depending on where exactly where the static pressure is measured. From Figure 64 above we can see that the $\mathrm{Cp}$ can change by about 0.25 per meter (roughly the length the test model).

The second form being the geometry is slightly different than then one being modeled on the computer. The differences could arise because the actual wind tunnel 
model was not measured; rather the original data points were used. The wind tunnel model used the original data points to make the solid model, which in turn machined the female mold. However, between the initial machining and the final product, there was sanding, shaping, and possible differences in thickness which may have led to a slightly different nose shape. Because a lack of time and resources we were not able to compare the actual build model to the computer simulation. It was believed that the geometry was not different enough to explain the differences.

Finally 3-D tunnel conditions were looked at to see if they had an effect on the radial pressure distribution. This is important because the pressure ports for the experimental data were on the right side of the model and there were no azimuthal ports to see if the flow was truly axisymmetric. This involved looking at what effect the rectangular tunnel cross section has and what effect model sideslip has.

A simple three dimensional computational model was made of the forebody attached to a faired over aft section. This was then ran inviscidly to solely look at if the pressure would vary and what kind of variation we might see. The first case was ran with the model straight in the rectangular tunnel to see if the tunnel walls. It was found that the rectangular cross section did not have an effect on the pressure distribution around the body. This can be seen in the cross section of the results at $20 \%$ chord in Figure 74 below. 

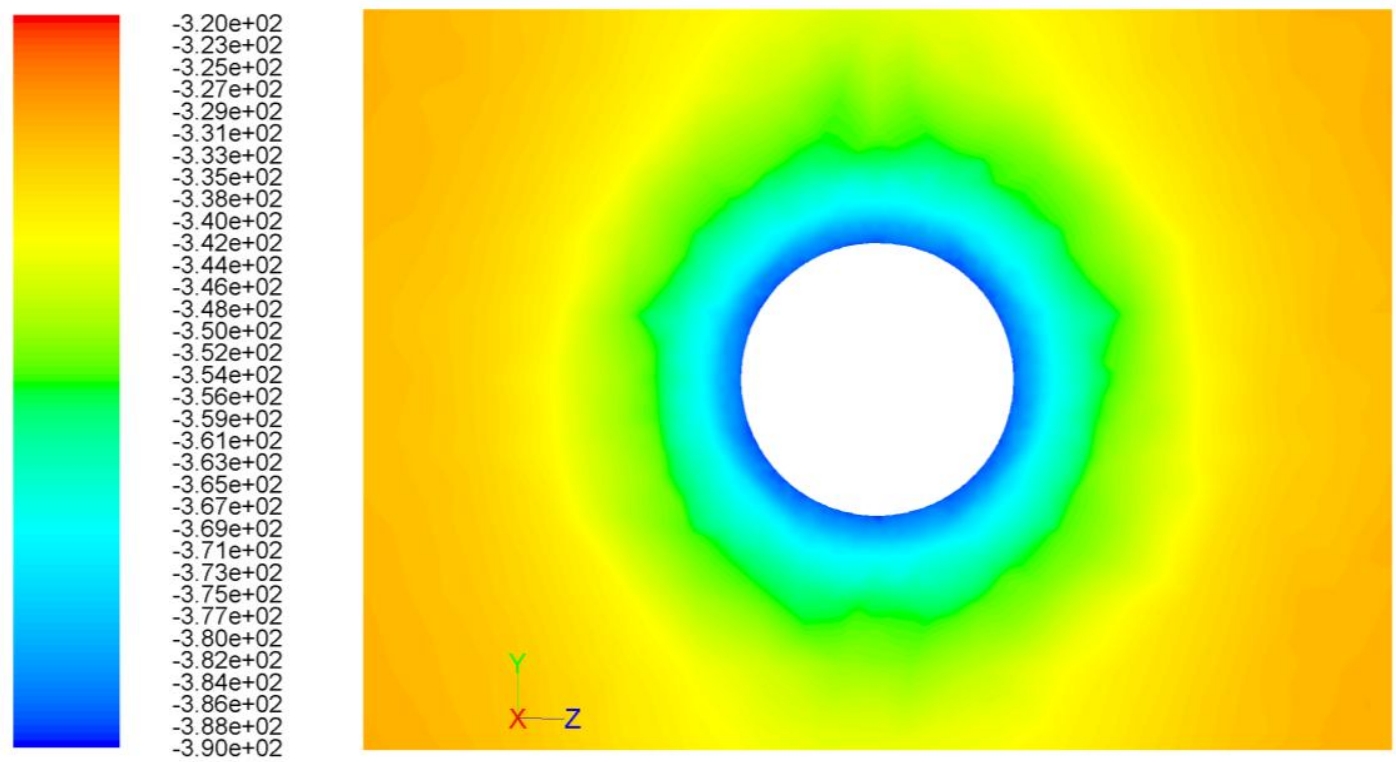

Figure $74: 3 \times 4 \mathrm{ft} 3-\mathrm{D}$ tunnel cross section static pressure contours for straight model at $20 \%$ chord

The computational model was then rotated to side slip angles of 2, 4, and 6 degrees to see how much that effected the pressure distribution. This had a large effect as seen in previous experiments (see Figure 11 for Goldschmied's rotation results). The effect of two degrees of sideslip can be seen in the flow field and around the body in Figure 75 below. This difference can be compared to the straight model to find how much the pressure changes for each degree of sideslip, all referenced at $20 \%$ chord. The correlation for small angles can be seen in Figure 76 below. Looking back to Figure 72 we see that there is about a 0.07 difference in $\mathrm{Cp}$ values between the experimental and CFD results, when looking at the plot we see that this corresponds to about 1.9 degree sideslip angle. This small sideslip angle could explain the difference between the CFD and the experimental result as there was no data to verify if the experimental model was seeing radially constant conditions. 

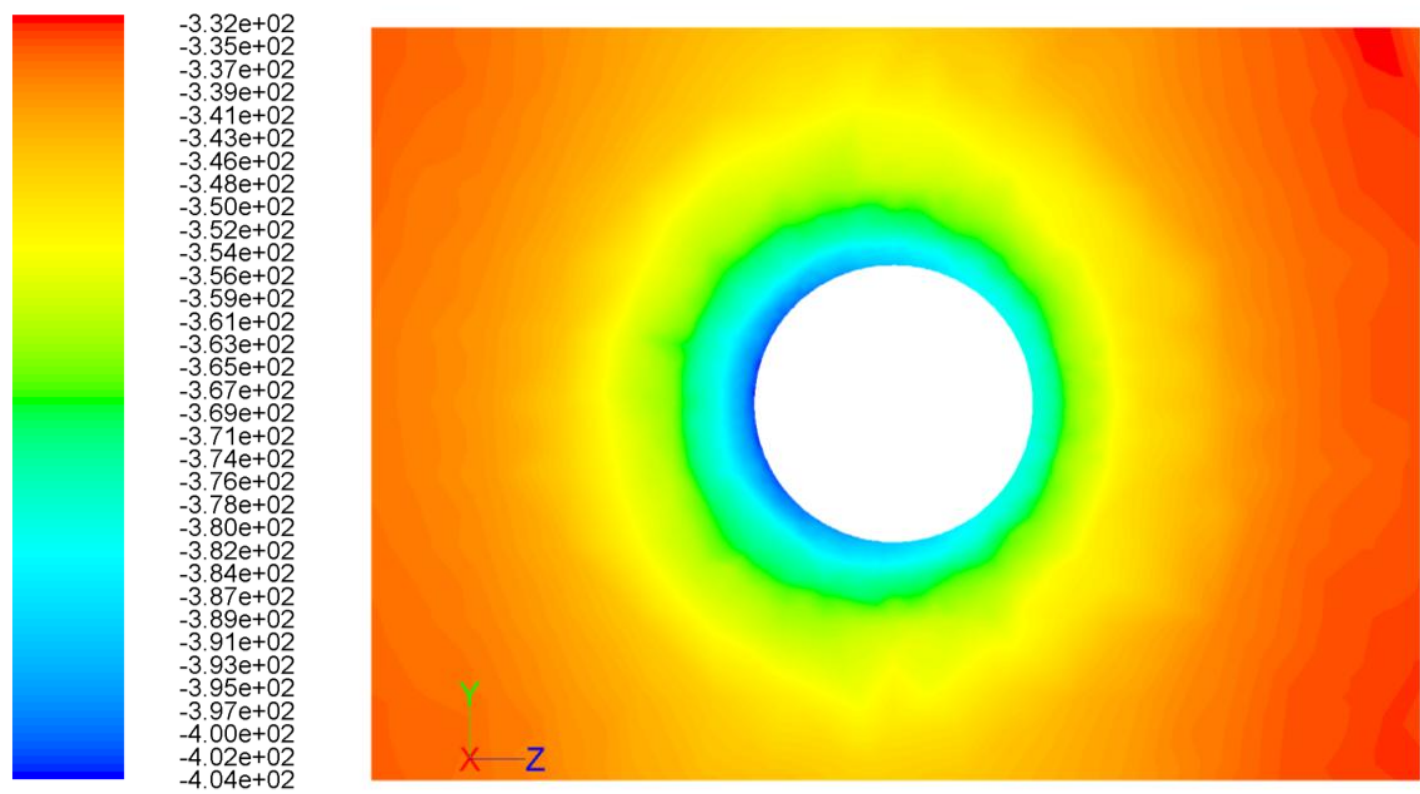

Figure 75: 3x4ft 3-D tunnel cross section static pressure contours with model in 2 degrees of beta at $20 \%$ chord

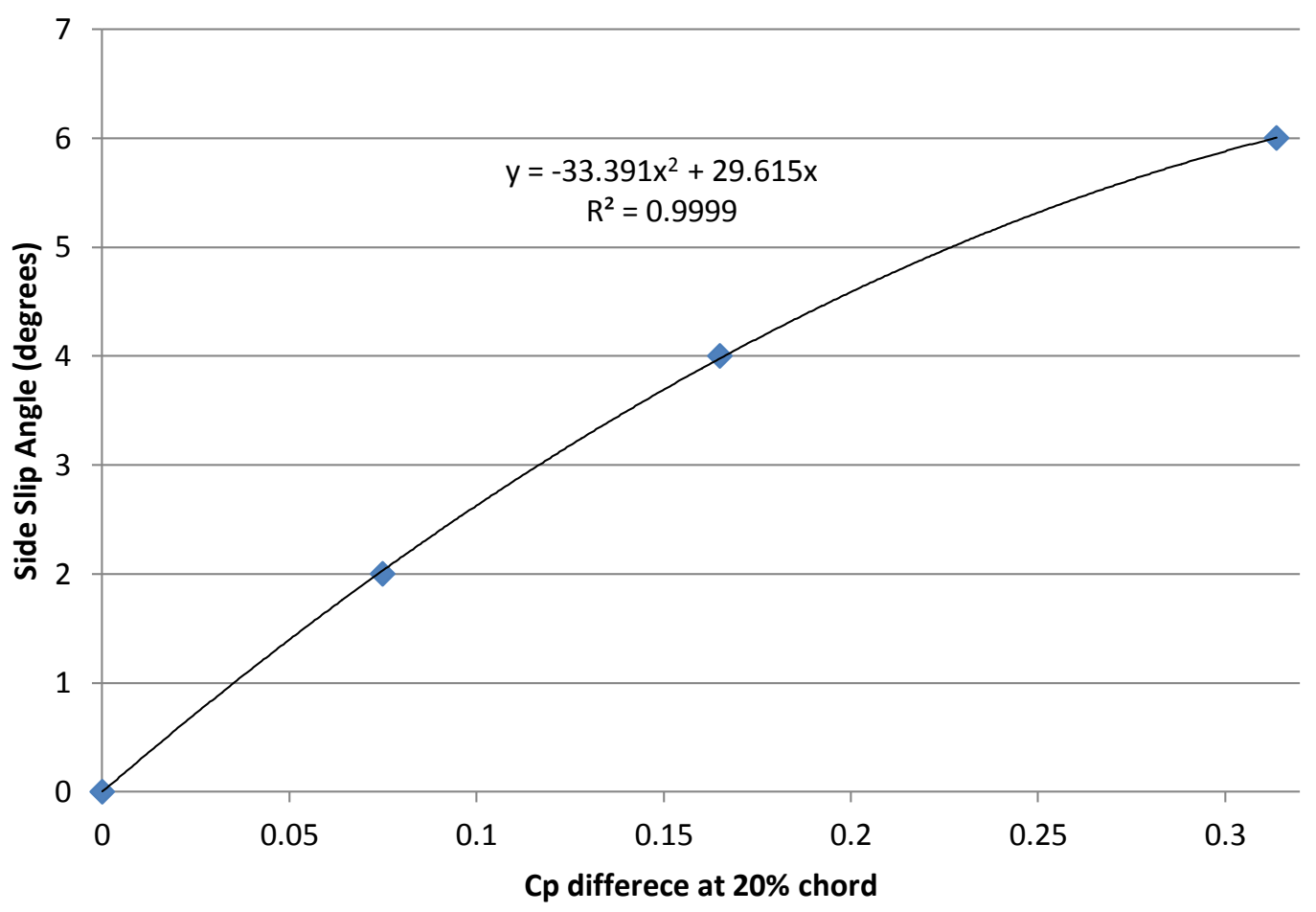

Figure 76: Correlation between $\mathrm{Cp}$ difference at $20 \%$ chord and side slip angle (beta) 
The pressure differences described above were present for all the fan settings, which can be seen in Figure 77 and in detail in Figure 78. These plots also show a large difference in the higher fan settings. The CFD took about $300 \mathrm{~Pa}$ fan difference for aft body reattachment and at $1500 \mathrm{~Pa}$ the aft section had a maximum pressure recovery of about 0.5 . For the wind tunnel model, there was not aft body reattachment until about $1629 \mathrm{~Pa}$ and then at the maximum fan setting of $2349 \mathrm{~Pa}$ the maximum pressure recovery was about 0.32 . The CFD significantly over-predicted the pressure recovery on the aft section and it did this at a much lower fan setting. When looking at these values compared to Goldschmied's and Peraudo et al. from Figure 19 on 24 above, the maximum pressure recovery that they achieved was around 0.55 , which is greater than all the values that we have observed here. This led us to question that the wind tunnel geometry or slot conditions were less than ideal, causing pressure recoveries that are not as high as they could be. Also it is noted that the aft geometry, as outlined in section 5.1.3 on page 44 , was modified from the SolidWorks model to make it a smoother shape and may partially explain some of the difference. This also did not rule out the possibility that the CFD model had a hard time predicting the separation point or pressure recovery. Looking at possible reasons, the first may be that the separation point may occur a little earlier on the tunnel model than on the CFD model. This would naturally require more fan power to get the flow to re-attach and turn around the corner. Secondly, if the lip on the aft section played a larger role than what CFD predicted we could expect to see lower aft body pressure recoveries. 


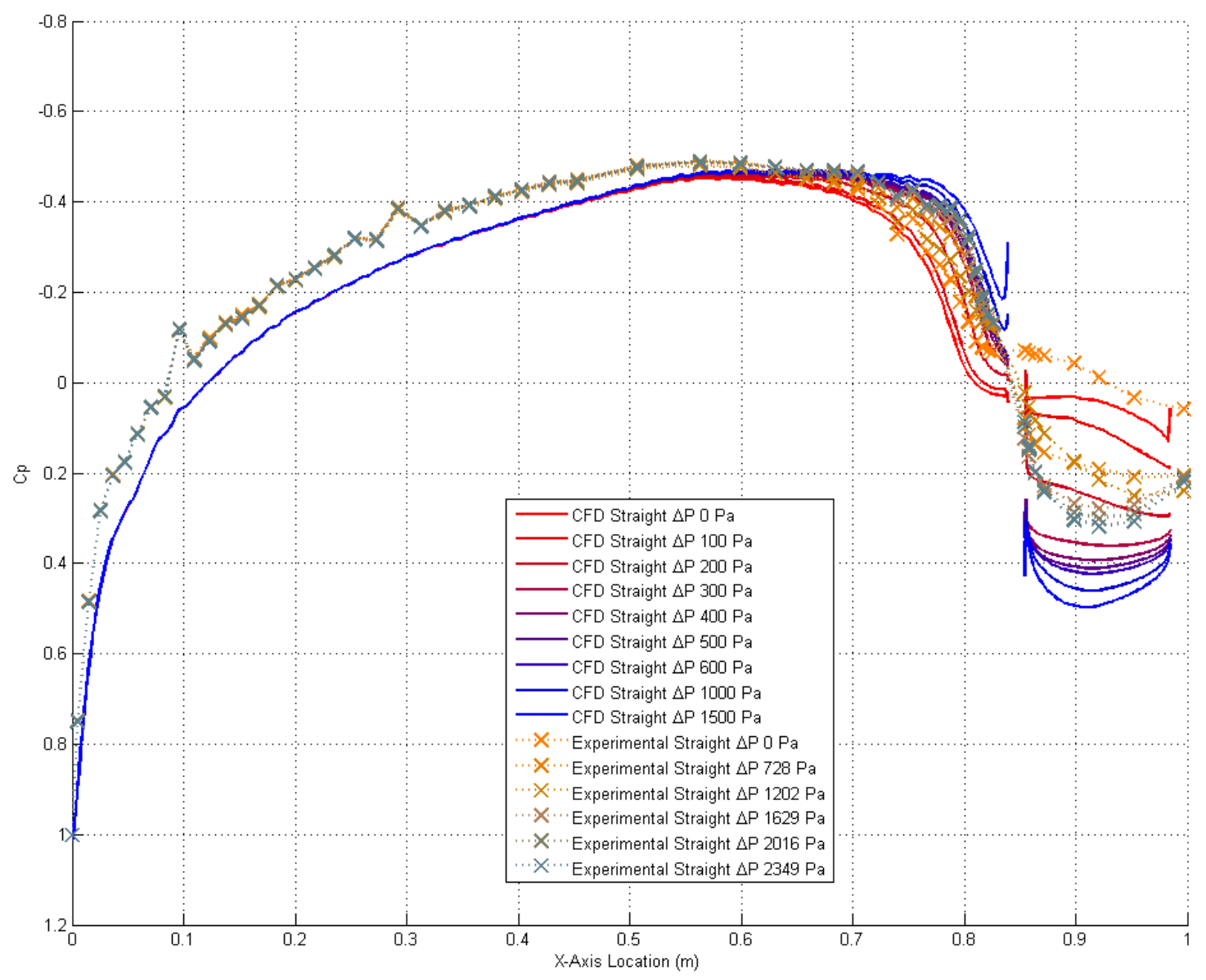

Figure 77: Comparison of Cp CFD data to Cal Poly experimental data for all fan conditions 


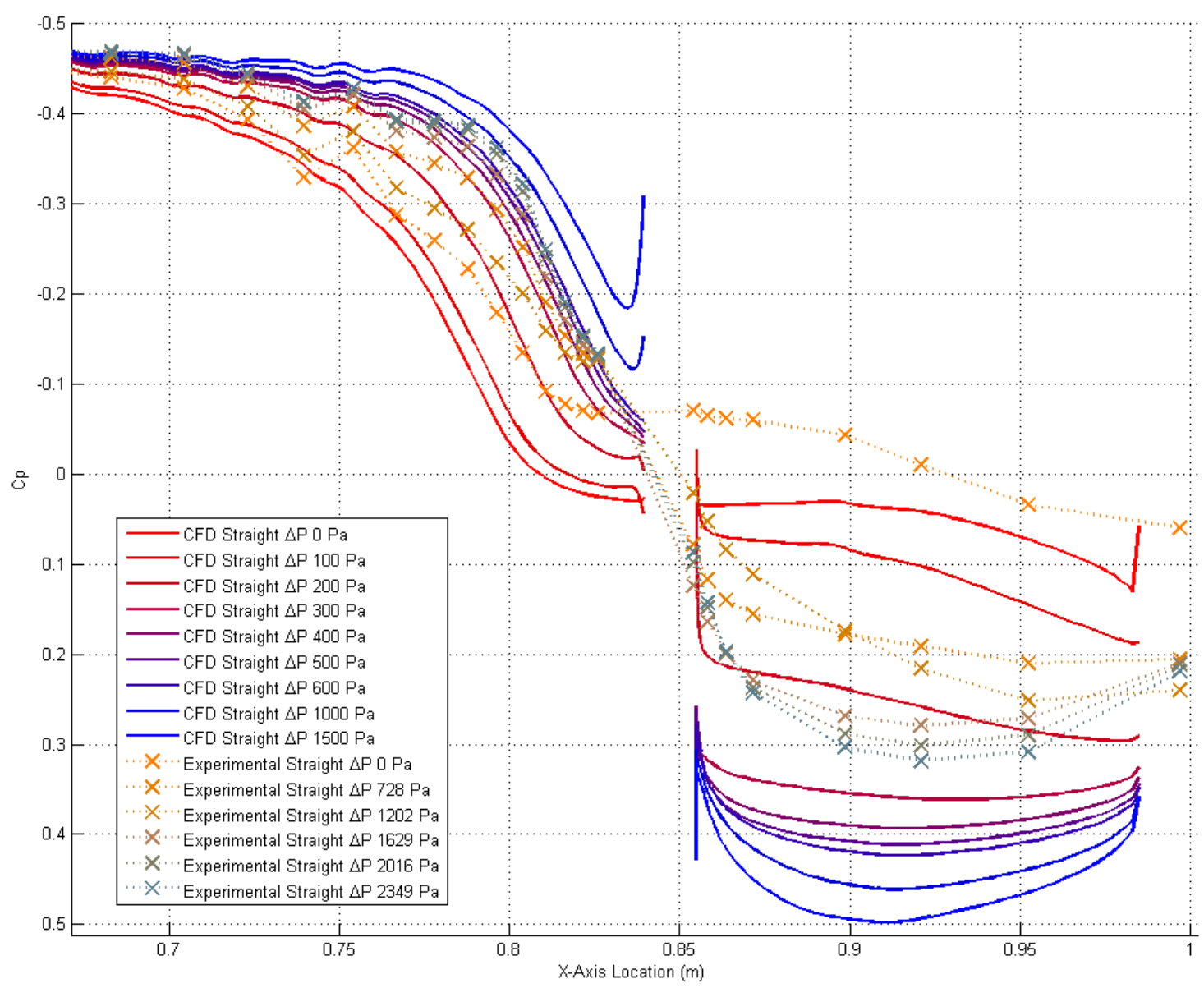

Figure 78: Comparison of CFD fan speeds to Cal Poly experimental data: Aft Section

When comparing the total drag of the CFD model to the wind tunnel model, it was difficult to get exact numbers because of the wide range of values measured from the load cell on the sting. Figure 79 shows the measured axial force values for the fan settings with different geometries. We assume that the drag values for the entire fan off settings would be pretty similar, as the changes in geometry were all small and in the separated region. The cusp and slot gap would be expected to have little effect in this region, and no clear trend could be observed. The large variations of the fan off conditions, going from the lowest drag coefficient of 0.043 to a maximum of 0.0714 and a single outlier of 0.1263 , appears to be an indication of the error involved with the data. 
The CFD results of 0.056 fall close to the middle of this range, but it is hard to find an error when there is not an exact value to compare to. From this data it seems that the CFD was able to predict the drag of the fan off; it is within the data spread form the test.

For the rest of the data, additional trends were indistinguishable, as many of the lines crossed over each other. Rather all we see is that the next axial force decreases as fan speed increases. This shows that the fan is able to add a propulsive force, but slot or cusp design have little influence. It seems that the differences that we are looking for have a smaller effect than the overall error in the experiment.

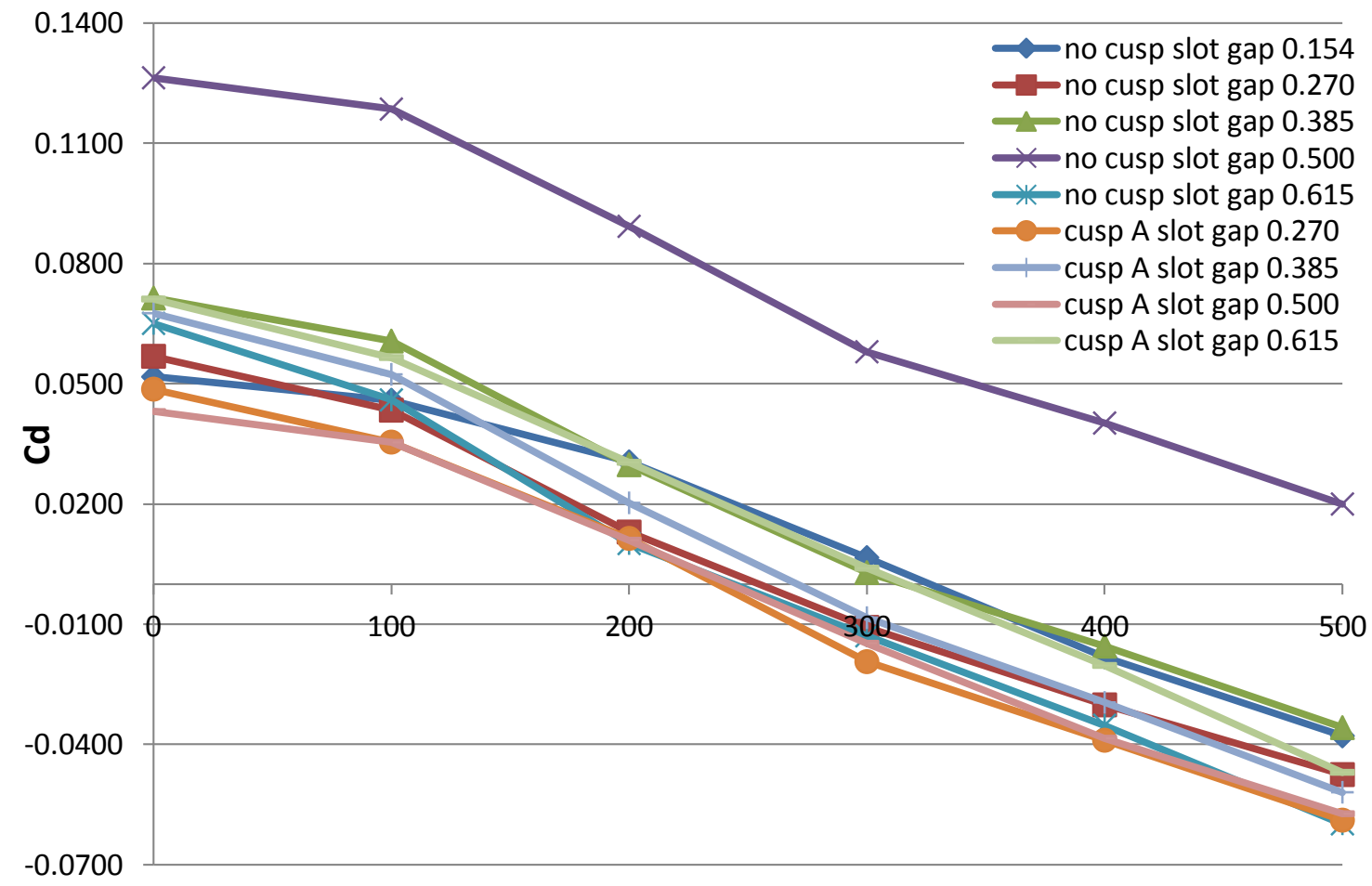

Fan Power (Watts)

Figure 79: Experimental axial force for different geometries at the 5 different fan settings 


\section{Conclusion}

When talking about the Goldschmied Propulsor to people, one of the main questions asked by many people is; "Does the Propulsor actually work? And if so are the gains enough to buy itself onto a vehicle?" From what Goldschmied originally claimed, up to a $50 \%$ reduction in drag from the most streamlined body, it seemed like it would have the potential to buy itself onto a vehicle. Through our research we were not able to see these gains. It actually turned out that the aft pressure recovery was counteracted by the a reduction in pressure leading up to the slot, making almost no difference in pressure drag when the fan was turned on. This shows that the smaller geometry tested in Cal Poly's wind tunnel was not able to replicate Goldschmied's claims of reduced pressure drag due to boundary layer ingestion.

On the other side, our research showed that the computational fluid dynamics approach was able to replicate most of the wind tunnel data. The drag forces were within the error bounds of the experimental data and the effect of the fan of the pressure distribution showed the same trends. The CFD and wind tunnel started to differ in the power needed for reattachment and the aft pressure recovery. In all cases, the CFD analysis required a great deal less fan pressure rise to yield aft body reattachment. The reason for this is not exactly known, rather it has been speculated that there are many factors that have ledto this large difference. Some factors include earlier separation on the main body, as earlier separation would take more effort to get to reattach. There were more losses in the suction slot on the real model then what was able to be modeled in an axisymmetric geometry. Also, a lot was learned from the different conditions ran. There was a large effect on the pressure distribution on the model due to the wind tunnel walls, but modeling the contraction or a rectangular cross section had little difference between those and the straight circular model. Additionally, the two or 
more equation turbulence models all produced similar results. From the grid independence study for the structured axisymmetric conditions there was small error $(1.63 \%)$ using the $\mathrm{GCl}$ method.

Finally, it seemed that the tunnel effects had a large effect on the aft body, and because of this the actual tunnel may accentuate what we saw going from freestream to the wind tunnel cases. In conclusion, it seems that the tested geometry does not produce the reduction in pressure drag we hoped for. There was potential for it to work, but would require an optimized geometry to remove the low pressure region leading up to the slot.

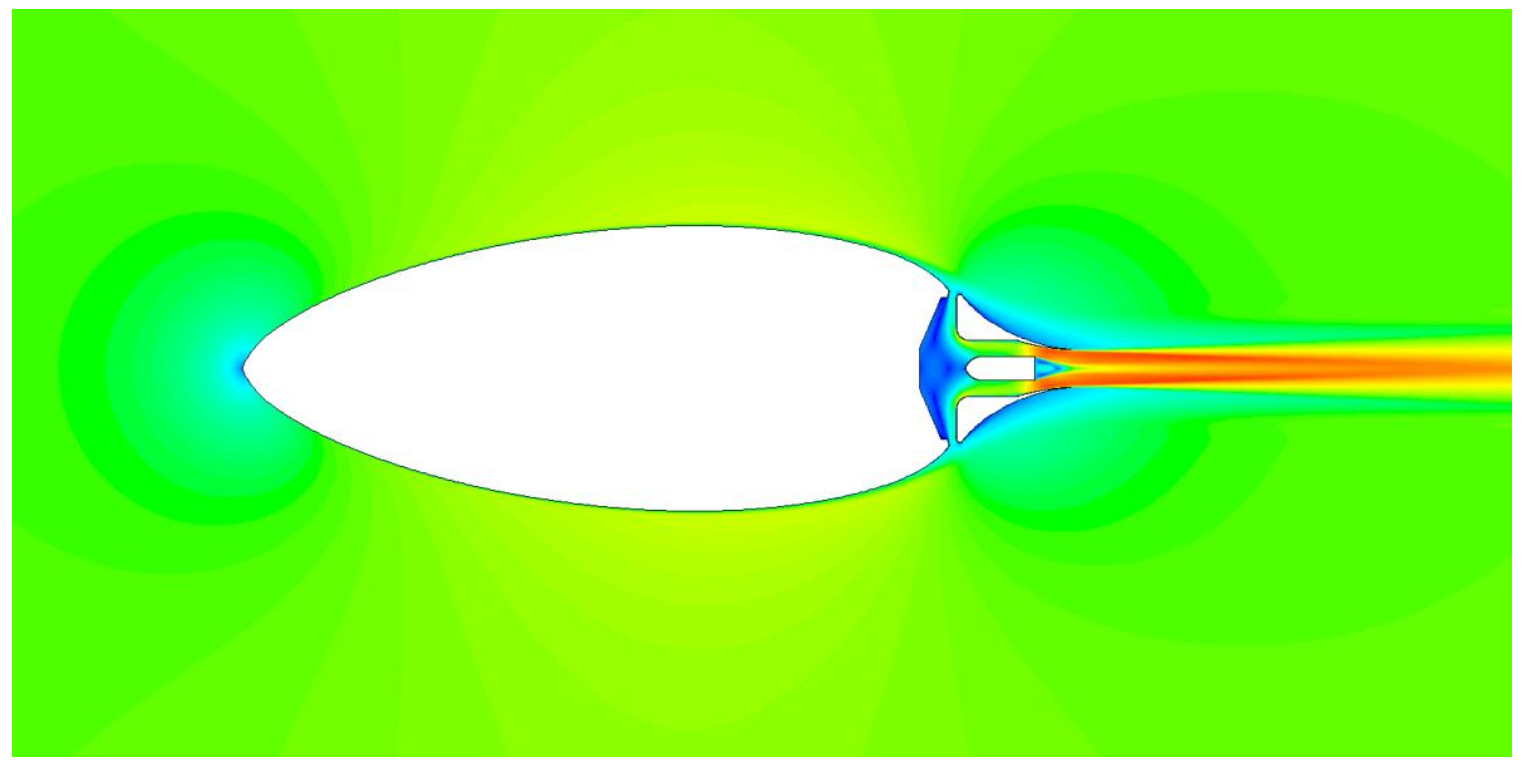

Figure 80: Flood filled velocity contours for max fan speed 


\section{Suggestions for Future Effort}

From the computational study there were a few items noticed that might make the test body perform better. It seemed that the shape was far from ideal and that a few changes may make a large difference with the aft pressure distribution.

1) Modify the geometry to keep the natural fan off separation point closer in front of the slot: This would reduce the suction need to keep the flow attached and minimize the low pressure region on the back side of the maximum thickness leading up to the slot and reduce the pressure drag that this low pressure region creates.

2) Make the back side to the slot entrance sharper: Currently it seems that the flow turns to enter the slot but the streamlines end up running into a flat section, which greatly disrupts the flow. This leads to difficulty in aft body re-attachment.

3) Make the entrance to the slot smoother: Instead of trying to turn the flow $90^{\circ}$ as it enters the slot, something more gradual with the goal of decreasing the suction needed to bring the flow to the fan.

4) Properly size motor and exit jet geometry: The current model uses an R/C motor and fan meant to be used in a high speed airplane, but it saw relatively slow moving air in the slot plenum. Because of this it is believed that the fan blades are not the correct pitch and are at least partially stalled for most of their operation. The exit geometry could be sized as either a diffuser or nozzle to get the optimal exit pressure and velocity to give the greatest propulsive efficiency. 


\section{References}

1. Goldschmied, Fabio. Wind Tunnel Test of the Modified Goldschmied Model with Propulsion and Empennage: Analysis of Test Results. FRG-82-1: David W. Taylor Naval Ship R\&D Center, 1982.

2. Goldschmied, Fabio R. On the Aerodynamic Optimization of Mini-RPV and Small GA Aircraft. AIAA 1984-2163-960 : 2nd applied Aerodynamics Conference, Seattle, WA, 1984.

3. Roskam, Jan and Fillman, Greg. Design for minimum fuselage drag. 0021-8669 vol.13 no.8 (639-640) : Journal of Aircraft, 1976.

4. Boeing Commercial Airplanes. Current Market Outlook 2011-2030. Seattle, WA : Boeing Market Anaylsis, 2011.

5. Bezos-O'Conner, Gaudy, et al. Fuel Efficiencies Through Airframe Improvements. AIAA 2011-3530 : 3rd AIAA Atmospheric Space Environments Conference, 2011.

6. Nicholai, L. M. and Carichner, G. E. Fundamentals of Aircraft and Airship Design. AIAA Educational Series : AIAA, Reston, VA, 2010.

7. Harshe, M., Pake, F. and Wasson, H. Investigation of A Boundary-Layer-Controlled Airship. Akron, Ohio : Goodyear Aircraft Corporation, 1957.

8. Cerreta, P. A. Wind-Tunnel Investigation of the Drag of a Proposed Boundary-Layer Controlled Airship. David W. Taylor Model Basin : Aero Rpt. 914, 1957.

9. Howe, Harvey. An Experimental Evaluation of a Low Propulsive Power, Discrete Suction Concept applied to an Axisymmetric Vehicle. David Taylop Naval Ship Research and Development Center : Bethesda, Maryland, 1982.

10. Goldschmied, Fabio. Fuselage Self-Propulsion by Static-Pressure Thrust: WindTunnel Verification. AIAA-87-2935 : AIAA/AHS/ASEE Aircraft Design, Systems and Operations Meeting, 1987.

11. Betz, A. History of Boundary Layer Contol in Germany. "Boundary Layer and Flow Control" by G.V. Lachmann : Pergamon Press, 1961. 
12. Richards, E. J., Walker, W. S. and Greening, J. R. Tests of a Griffith Airfoil in the 13ft x 9ft Wind Tunnel. Ministry of Supply, London: His Majesty's Stationary Office : Aeronautical Research Council Reports and Memoranda, 1944.

13. Goldschmied, Fabio R. Aerodynamic Hull Design for HASPA LTA Optimization. AIAA-58418-412 : American Institute of Aerodynamics and Austronautics, 1978.

14. Ringleb, Friedrich. Seperation Control by Trapped Vortices. Boundary-Layer and Flow Control, edited by G.V. Lachmann, Vol. I : Pergamon Press, New York, 1961.

15. Goldschmied, Fabio. Jet-Propulsion of Subsonic Bodies with Jet Total-Heal Equal to Free Stream's. AIAA-83-1790 : AIAA Apploed Aerodynamics Conference, July 1315 th, 1983.

16. Peraudo, P. N., Schetz, J. A. and Roy, C. J. Computational Study of the Embedded Engine Static Pressure Thrust Propulsion System. Nashville, Tennessee : 50th AIAA Aerospace Sciences Meeting including the New Horizons Forum and Aerospace Exposition, January 2012.

17. Roepke, Joshua. An Investigation of a Goldschmied Propulsor. Masters Thesis: California Polytechnic State University, San Luis Obispo, 2012.

18. Thomason, Nicole. Experimental Investigation of Suction Geometry on a Goldschmied Propulsor. Masters Thesis: California Polytechnic State University, San Luis Obispo, 2012.

19. Altmann, Greg. An Investigative Study of Gurney Flaps on a NACA 0036 Airfoil. California Polytechnic State University Aerospace Engineering : Masters Thesis, 2011.

20. Thomas, Mathew L., Pandey, Dorian V. and Nguyen, Jason N. Flow Characteristics of the Renovated Cal Poly $3 \times 4$ ft Subsonic Wind Tunnel. Senior Project : California Polytechnic State University, San Luis Obispo, 2011.

21. Goldschmied, Fabio. Integrated Hull Design, Boundary-Layer Control, and Propulsion of Submerged Bodies. AIAA-1699-658: Second Propulsion Joint Specialist Conference, Colorado Springs, CO, 1966. 
22. Crowe, Clayton, et al. Engineering Fluid Mechanics. R.R. Donnelley/Jefferson City : John Wiley \& Sons, INC, 2009.

23. White, Frank. Viscous Fluid Flow. New York, NY : McGraw-Hill, 2006.

24. Celik, Ismail, et al. Procedure for Estimation and Reporting of Uncertainty Due to Discritization in CFD Applications. Journal of Fluid Mechanics: Volume 130, July, 2008.

25. Oberkampf, William and Roy, Christopher. Verification adn Validation in Scientific Computing. Cambridge, New York : Cambridge University Press, 2010.

26. Tannehill, John, Anderson, Dale and Pletcher, Richard. Computational Fluid Mechanics and Heat Transfer. Philadelphia, PA : Taylor \& Francis, 1997.

27. ANSYS. ANSYS FLUENT Theory Guide. Release 13.0 : s.n., November, 2010.

28. ANSYSS. ANSYS FLUENT User's Guide. Release 13.0 : s.n., November 2010.

29. Wilcox, David. Turbulence Modeling for CFD. San Diego, CA : Birmingham Press, 2004. 


\section{Appendix A: Model Geometry Points}

\section{Forebody Geometry Points:}

\begin{tabular}{|c|c|c|}
\hline$x$ & $Y$ & Z \\
\hline $0.0000 E+00$ & $-1.2382 \mathrm{E}-07$ & $0.0000 E+00$ \\
\hline $1.0000 \mathrm{E}-02$ & $1.8893 \mathrm{E}-02$ & $0.0000 \mathrm{E}+00$ \\
\hline $2.0000 \mathrm{E}-02$ & 3.0980E-02 & $0.0000 E+00$ \\
\hline $3.0000 \mathrm{E}-02$ & $3.9956 \mathrm{E}-02$ & $0.0000 E+00$ \\
\hline $4.0000 \mathrm{E}-02$ & $4.7498 \mathrm{E}-02$ & $0.0000 \mathrm{E}+00$ \\
\hline $5.0000 \mathrm{E}-02$ & 5.4294E-02 & $0.0000 E+00$ \\
\hline $6.0000 \mathrm{E}-02$ & $6.0594 \mathrm{E}-02$ & $0.0000 E+00$ \\
\hline 7.0000E-02 & $6.6483 \mathrm{E}-02$ & $0.0000 \mathrm{E}+00$ \\
\hline $8.0000 \mathrm{E}-02$ & 7.1999E-02 & $0.0000 E+00$ \\
\hline $9.0000 \mathrm{E}-02$ & 7.7177E-02 & $0.0000 E+00$ \\
\hline $1.0000 \mathrm{E}-01$ & $8.2056 \mathrm{E}-02$ & $0.0000 E+00$ \\
\hline $1.1000 \mathrm{E}-01$ & $8.6676 \mathrm{E}-02$ & $0.0000 E+00$ \\
\hline $1.2000 \mathrm{E}-01$ & $9.1072 \mathrm{E}-02$ & $0.0000 \mathrm{E}+00$ \\
\hline $1.3000 \mathrm{E}-01$ & $9.5271 \mathrm{E}-02$ & $0.0000 E+00$ \\
\hline $1.4000 \mathrm{E}-01$ & $9.9292 \mathrm{E}-02$ & $0.0000 E+00$ \\
\hline $1.5000 \mathrm{E}-01$ & $1.0315 \mathrm{E}-01$ & $0.0000 \mathrm{E}+00$ \\
\hline $1.6000 \mathrm{E}-01$ & $1.0684 \mathrm{E}-01$ & $0.0000 E+00$ \\
\hline $1.7000 \mathrm{E}-01$ & $1.1039 \mathrm{E}-01$ & $0.0000 E+00$ \\
\hline $1.8000 \mathrm{E}-01$ & $1.1379 \mathrm{E}-01$ & $0.0000 \mathrm{E}+00$ \\
\hline $1.9000 \mathrm{E}-01$ & $1.1705 \mathrm{E}-01$ & $0.0000 E+00$ \\
\hline $2.0000 \mathrm{E}-01$ & $1.2018 \mathrm{E}-01$ & $0.0000 E+00$ \\
\hline $2.1000 \mathrm{E}-01$ & $1.2318 \mathrm{E}-01$ & $0.0000 \mathrm{E}+00$ \\
\hline $2.2000 \mathrm{E}-01$ & $1.2607 \mathrm{E}-01$ & $0.0000 \mathrm{E}+00$ \\
\hline $2.3000 \mathrm{E}-01$ & $1.2884 \mathrm{E}-01$ & $0.0000 \mathrm{E}+00$ \\
\hline $2.4000 \mathrm{E}-01$ & $1.3152 \mathrm{E}-01$ & $0.0000 E+00$ \\
\hline $2.5000 \mathrm{E}-01$ & $1.3408 \mathrm{E}-01$ & $0.0000 E+00$ \\
\hline $2.6000 \mathrm{E}-01$ & $1.3655 \mathrm{E}-01$ & $0.0000 \mathrm{E}+00$ \\
\hline $2.7000 \mathrm{E}-01$ & $1.3893 \mathrm{E}-01$ & $0.0000 E+00$ \\
\hline $2.8000 \mathrm{E}-01$ & $1.4120 \mathrm{E}-01$ & $0.0000 E+00$ \\
\hline $2.9000 \mathrm{E}-01$ & $1.4338 \mathrm{E}-01$ & $0.0000 \mathrm{E}+00$ \\
\hline $3.0000 \mathrm{E}-01$ & 1.4547E-01 & $0.0000 E+00$ \\
\hline $3.1000 \mathrm{E}-01$ & $1.4746 \mathrm{E}-01$ & $0.0000 E+00$ \\
\hline $3.2000 \mathrm{E}-01$ & $1.4936 \mathrm{E}-01$ & $0.0000 \mathrm{E}+00$ \\
\hline $3.3000 \mathrm{E}-01$ & 1.5117E-01 & $0.0000 \mathrm{E}+00$ \\
\hline $3.4000 \mathrm{E}-01$ & $1.5290 \mathrm{E}-01$ & $0.0000 E+00$ \\
\hline $3.5000 \mathrm{E}-01$ & $1.5454 \mathrm{E}-01$ & $0.0000 E+00$ \\
\hline $3.6000 \mathrm{E}-01$ & $1.5609 \mathrm{E}-01$ & $0.0000 E+00$ \\
\hline $3.7000 \mathrm{E}-01$ & $1.5757 \mathrm{E}-01$ & $0.0000 E+00$ \\
\hline $3.8000 \mathrm{E}-01$ & $1.5896 \mathrm{E}-01$ & $0.0000 E+00$ \\
\hline $3.9000 \mathrm{E}-01$ & 1.6027E-01 & $0.0000 E+00$ \\
\hline $4.0000 \mathrm{E}-01$ & $1.6150 \mathrm{E}-01$ & $0.0000 \mathrm{E}+00$ \\
\hline 4.1000E-01 & $1.6265 \mathrm{E}-01$ & $0.0000 E+00$ \\
\hline $4.2000 \mathrm{E}-01$ & $1.6372 \mathrm{E}-01$ & $0.0000 E+00$ \\
\hline $4.3000 \mathrm{E}-01$ & $1.6471 \mathrm{E}-01$ & $0.0000 E+00$ \\
\hline 4.4000E-01 & $1.6562 \mathrm{E}-01$ & $0.0000 E+00$ \\
\hline $4.5000 \mathrm{E}-01$ & $1.6645 \mathrm{E}-01$ & $0.0000 E+00$ \\
\hline $4.6000 \mathrm{E}-01$ & 1.6719E-01 & $0.0000 E+00$ \\
\hline 4.7000E-01 & $1.6786 \mathrm{E}-01$ & $0.0000 E+00$ \\
\hline $4.8000 \mathrm{E}-01$ & $1.6843 \mathrm{E}-01$ & $0.0000 E+00$ \\
\hline
\end{tabular}




\begin{tabular}{|l|l|l|}
\hline $4.9000 \mathrm{E}-01$ & $1.6893 \mathrm{E}-01$ & $0.0000 \mathrm{E}+00$ \\
\hline $5.0000 \mathrm{E}-01$ & $1.6933 \mathrm{E}-01$ & $0.0000 \mathrm{E}+00$ \\
\hline $5.1000 \mathrm{E}-01$ & $1.6965 \mathrm{E}-01$ & $0.0000 \mathrm{E}+00$ \\
\hline $5.2000 \mathrm{E}-01$ & $1.6987 \mathrm{E}-01$ & $0.0000 \mathrm{E}+00$ \\
\hline $5.3000 \mathrm{E}-01$ & $1.7000 \mathrm{E}-01$ & $0.0000 \mathrm{E}+00$ \\
\hline $5.4000 \mathrm{E}-01$ & $1.7003 \mathrm{E}-01$ & $0.000 \mathrm{E}+00$ \\
\hline $5.5000 \mathrm{E}-01$ & $1.6995 \mathrm{E}-01$ & $0.000 \mathrm{E}+00$ \\
\hline $5.6000 \mathrm{E}-01$ & $1.6977 \mathrm{E}-01$ & $0.0000 \mathrm{E}+00$ \\
\hline $5.7000 \mathrm{E}-01$ & $1.6949 \mathrm{E}-01$ & $0.0000 \mathrm{E}+00$ \\
\hline $5.8000 \mathrm{E}-01$ & $1.6909 \mathrm{E}-01$ & $0.0000 \mathrm{E}+00$ \\
\hline $5.9000 \mathrm{E}-01$ & $1.6859 \mathrm{E}-01$ & $0.0000 \mathrm{E}+00$ \\
\hline $6.0000 \mathrm{E}-01$ & $1.6798 \mathrm{E}-01$ & $0.0000 \mathrm{E}+00$ \\
\hline $6.1000 \mathrm{E}-01$ & $1.6727 \mathrm{E}-01$ & $0.0000 \mathrm{E}+00$ \\
\hline $6.2000 \mathrm{E}-01$ & $1.6644 \mathrm{E}-01$ & $0.0000 \mathrm{E}+00$ \\
\hline $6.3000 \mathrm{E}-01$ & $1.6551 \mathrm{E}-01$ & $0.000 \mathrm{E}+00$ \\
\hline $6.4000 \mathrm{E}-01$ & $1.6446 \mathrm{E}-01$ & $0.0000 \mathrm{E}+00$ \\
\hline $6.5000 \mathrm{E}-01$ & $1.6329 \mathrm{E}-01$ & $0.0000 \mathrm{E}+00$ \\
\hline $6.6000 \mathrm{E}-01$ & $1.6201 \mathrm{E}-01$ & $0.0000 \mathrm{E}+00$ \\
\hline $6.7000 \mathrm{E}-01$ & $1.6059 \mathrm{E}-01$ & $0.0000 \mathrm{E}+00$ \\
\hline $6.8000 \mathrm{E}-01$ & $1.5903 \mathrm{E}-01$ & $0.0000 \mathrm{E}+00$ \\
\hline $6.9000 \mathrm{E}-01$ & $1.5732 \mathrm{E}-01$ & $0.0000 \mathrm{E}+00$ \\
\hline $7.0000 \mathrm{E}-01$ & $1.5546 \mathrm{E}-01$ & $0.0000 \mathrm{E}+00$ \\
\hline $7.1000 \mathrm{E}-01$ & $1.5344 \mathrm{E}-01$ & $0.000 \mathrm{E}+00$ \\
\hline $7.2000 \mathrm{E}-01$ & $1.5124 \mathrm{E}-01$ & $0.0000 \mathrm{E}+00$ \\
\hline $7.3000 \mathrm{E}-01$ & $1.4886 \mathrm{E}-01$ & $0.0000 \mathrm{E}+00$ \\
\hline $7.4000 \mathrm{E}-01$ & $1.4628 \mathrm{E}-01$ & $0.0000 \mathrm{E}+00$ \\
\hline $7.5000 \mathrm{E}-01$ & $1.4346 \mathrm{E}-01$ & $0.0000 \mathrm{E}+00$ \\
\hline $7.6000 \mathrm{E}-01$ & $1.4038 \mathrm{E}-01$ & $0.0000 \mathrm{E}+00$ \\
\hline $7.7000 \mathrm{E}-01$ & $1.3698 \mathrm{E}-01$ & $0.0000 \mathrm{E}+00$ \\
\hline $7.8000 \mathrm{E}-01$ & $1.3318 \mathrm{E}-01$ & $0.0000 \mathrm{E}+00$ \\
\hline $7.9000 \mathrm{E}-01$ & $1.2891 \mathrm{E}-01$ & $0.0000 \mathrm{E}+00$ \\
\hline $8.0000 \mathrm{E}-01$ & $1.2404 \mathrm{E}-01$ & $0.0000 \mathrm{E}+00$ \\
\hline $8.1000 \mathrm{E}-01$ & $1.1840 \mathrm{E}-01$ & $0.000 \mathrm{E}+00$ \\
\hline $8.2000 \mathrm{E}-01$ & $1.1168 \mathrm{E}-01$ & $0.0000 \mathrm{E}+00$ \\
\hline $8.3000 \mathrm{E}-01$ & $1.0327 \mathrm{E}-01$ & $0.0000 \mathrm{E}+00$ \\
\hline
\end{tabular}

\section{Cusp and Front Geometry Points:}

\begin{tabular}{|c|c|c|}
\hline$X$ & $Y$ & $Z$ \\
\hline $8.3626 \mathrm{E}-01$ & $9.6637 \mathrm{E}-02$ & $0.0000 \mathrm{E}+00$ \\
\hline $8.3772 \mathrm{E}-01$ & $9.4934 \mathrm{E}-02$ & $0.0000 \mathrm{E}+00$ \\
\hline $8.3851 \mathrm{E}-01$ & $9.3633 \mathrm{E}-02$ & $0.0000 \mathrm{E}+00$ \\
\hline $8.3933 \mathrm{E}-01$ & $9.1948 \mathrm{E}-02$ & $0.0000 \mathrm{E}+00$ \\
\hline $8.3882 \mathrm{E}-01$ & $9.1069 \mathrm{E}-02$ & $0.0000 \mathrm{E}+00$ \\
\hline $8.3846 \mathrm{E}-01$ & $9.0014 \mathrm{E}-02$ & $0.0000 \mathrm{E}+00$ \\
\hline $8.3825 \mathrm{E}-01$ & $8.9186 \mathrm{E}-02$ & $0.0000 \mathrm{E}+00$ \\
\hline $8.3808 \mathrm{E}-01$ & $8.7860 \mathrm{E}-02$ & $0.0000 \mathrm{E}+00$ \\
\hline $8.3805 \mathrm{E}-01$ & $8.6425 \mathrm{E}-02$ & $0.0000 \mathrm{E}+00$ \\
\hline $8.3806 \mathrm{E}-01$ & $8.5058 \mathrm{E}-02$ & $0.0000 \mathrm{E}+00$ \\
\hline $8.3476 \mathrm{E}-01$ & $8.5090 \mathrm{E}-02$ & $0.0000 \mathrm{E}+00$ \\
\hline $8.2996 \mathrm{E}-01$ & $8.5090 \mathrm{E}-02$ & $0.0000 \mathrm{E}+00$ \\
\hline $8.0380 \mathrm{E}-01$ & $2.4638 \mathrm{E}-02$ & $0.0000 \mathrm{E}+00$ \\
\hline $8.0380 \mathrm{E}-01$ & $0.0000 \mathrm{E}+00$ & $0.0000 \mathrm{E}+00$ \\
\hline
\end{tabular}




\section{Aftbody and Fan Points:}

\begin{tabular}{|c|c|c|}
\hline$X$ & $Y$ & $Z$ \\
\hline $8.5719 \mathrm{E}-01$ & $0.0000 \mathrm{E}+00$ & $0.0000 \mathrm{E}+00$ \\
\hline $8.6319 \mathrm{E}-01$ & $1.0000 \mathrm{E}-02$ & $0.0000 \mathrm{E}+00$ \\
\hline $8.7319 \mathrm{E}-01$ & $1.5000 \mathrm{E}-02$ & $0.0000 \mathrm{E}+00$ \\
\hline $8.7319 \mathrm{E}-01$ & $3.4500 \mathrm{E}-02$ & $0.0000 \mathrm{E}+00$ \\
\hline $8.6219 \mathrm{E}-01$ & $3.4500 \mathrm{E}-02$ & $0.0000 \mathrm{E}+00$ \\
\hline $8.5825 \mathrm{E}-01$ & $3.5019 \mathrm{E}-02$ & $0.0000 \mathrm{E}+00$ \\
\hline $8.5457 \mathrm{E}-01$ & $3.6542 \mathrm{E}-02$ & $0.0000 \mathrm{E}+00$ \\
\hline $8.5141 \mathrm{E}-01$ & $3.8964 \mathrm{E}-02$ & $0.0000 \mathrm{E}+00$ \\
\hline $8.4899 \mathrm{E}-01$ & $4.2120 \mathrm{E}-02$ & $0.0000 \mathrm{E}+00$ \\
\hline $8.4747 \mathrm{E}-01$ & $4.5796 \mathrm{E}-02$ & $0.0000 \mathrm{E}+00$ \\
\hline $8.4695 \mathrm{E}-01$ & $4.9740 \mathrm{E}-02$ & $0.0000 \mathrm{E}+00$ \\
\hline $8.4695 \mathrm{E}-01$ & $8.5560 \mathrm{E}-02$ & $0.0000 \mathrm{E}+00$ \\
\hline $8.4704 \mathrm{E}-01$ & $8.6217 \mathrm{E}-02$ & $0.0000 \mathrm{E}+00$ \\
\hline $8.4729 \mathrm{E}-01$ & $8.6830 \mathrm{E}-02$ & $0.0000 \mathrm{E}+00$ \\
\hline $8.4769 \mathrm{E}-01$ & $8.7356 \mathrm{E}-02$ & $0.0000 \mathrm{E}+00$ \\
\hline $8.4822 \mathrm{E}-01$ & $8.7760 \mathrm{E}-02$ & $0.0000 \mathrm{E}+00$ \\
\hline $8.4883 \mathrm{E}-01$ & $8.8013 \mathrm{E}-02$ & $0.0000 \mathrm{E}+00$ \\
\hline $8.4949 \mathrm{E}-01$ & $8.8100 \mathrm{E}-02$ & $0.0000 \mathrm{E}+00$ \\
\hline $8.5269 \mathrm{E}-01$ & $8.8100 \mathrm{E}-02$ & $0.0000 \mathrm{E}+00$ \\
\hline $8.6299 \mathrm{E}-01$ & $7.6800 \mathrm{E}-02$ & $0.0000 \mathrm{E}+00$ \\
\hline $8.7709 \mathrm{E}-01$ & $6.3800 \mathrm{E}-02$ & $0.0000 \mathrm{E}+00$ \\
\hline $8.8589 \mathrm{E}-01$ & $5.7500 \mathrm{E}-02$ & $0.0000 \mathrm{E}+00$ \\
\hline $8.9469 \mathrm{E}-01$ & $5.2200 \mathrm{E}-02$ & $0.0000 \mathrm{E}+00$ \\
\hline $9.0349 \mathrm{E}-01$ & $4.6900 \mathrm{E}-02$ & $0.0000 \mathrm{E}+00$ \\
\hline $9.2109 \mathrm{E}-01$ & $3.8800 \mathrm{E}-02$ & $0.0000 \mathrm{E}+00$ \\
\hline $9.4759 \mathrm{E}-01$ & $2.9700 \mathrm{E}-02$ & $0.0000 \mathrm{E}+00$ \\
\hline $9.8279 \mathrm{E}-01$ & $2.3800 \mathrm{E}-02$ & $0.0000 \mathrm{E}+00$ \\
\hline $9.4869 \mathrm{E}-01$ & $2.7000 \mathrm{E}-02$ & $0.0000 \mathrm{E}+00$ \\
\hline $9.1869 \mathrm{E}-01$ & $3.4500 \mathrm{E}-02$ & $0.0000 \mathrm{E}+00$ \\
\hline $8.8869 \mathrm{E}-01$ & $3.4500 \mathrm{E}-02$ & $0.0000 \mathrm{E}+00$ \\
\hline $8.8869 \mathrm{E}-01$ & $1.5000 \mathrm{E}-02$ & $0.0000 \mathrm{E}+00$ \\
\hline $9.1869 \mathrm{E}-01$ & $1.5000 \mathrm{E}-02$ & $0.0000 \mathrm{E}+00$ \\
\hline $9.4869 \mathrm{E}-01$ & $1.5000 \mathrm{E}-02$ & $0.0000 \mathrm{E}+00$ \\
\hline $9.4869 \mathrm{E}-01$ & $0.0000 \mathrm{E}+00$ & $0.0000 \mathrm{E}+00$ \\
\hline & & \\
\hline & & \\
\hline
\end{tabular}

